\title{
The Standardization Of Outpatient Procedure (STOP) Narcotics: A Prospective Non-Inferiority Study To Reduce Opioid Use In Outpatient General Surgical Procedures
}

Luke B. Hartford, The University of Western Ontario

Supervisor: Van Koughnett, Julie Ann, The University of Western Ontario

: Leslie, Ken, The University of Western Ontario

: Brackstone, Muriel, The University of Western Ontario

A thesis submitted in partial fulfillment of the requirements for the Master of Science degree in Surgery

(C) Luke B. Hartford 2019

Follow this and additional works at: https://ir.lib.uwo.ca/etd

Part of the Surgery Commons

\section{Recommended Citation}

Hartford, Luke B., "The Standardization Of Outpatient Procedure (STOP) Narcotics: A Prospective NonInferiority Study To Reduce Opioid Use In Outpatient General Surgical Procedures" (2019). Electronic Thesis and Dissertation Repository. 6589.

https://ir.lib.uwo.ca/etd/6589

This Dissertation/Thesis is brought to you for free and open access by Scholarship@Western. It has been accepted for inclusion in Electronic Thesis and Dissertation Repository by an authorized administrator of Scholarship@Western. For more information, please contact wlswadmin@uwo.ca. 


\begin{abstract}
:
Surgeons play a significant role in the opioid crisis. A standardized, multipronged analgesia protocol was implemented with the objective of providing adequate analgesia while reducing opioids after outpatient breast and anorectal surgery.
\end{abstract}

A prospective, pre- and post-intervention study was designed to demonstrate non-inferiority for patient-reported post-operative pain. The intervention included patient and provider education, and multimodal perioperative analgesic strategies including opioid rescue prescriptions.

We evaluated 266 procedures. After implementation of the STOP Narcotics protocol, average post-operative pain scores in the post-intervention groups was non-inferior compared to the preintervention groups [(2.0 vs $2.1 / 10, \mathrm{p}=0.40$, breast procedures) and $(2.8$ vs $2.6 / 10, \mathrm{p}=0.33$, anorectal procedures)]. Opioid prescribing decreased by $50 \%$. Only $45 \%$ of patients filled their rescue opioid prescriptions.

A standardized pain care bundle significantly decreased opioid prescribing while adequately treating post-operative pain. This initiative provides a framework for future guidelines in outpatient breast and anorectal surgery.

Key Words: Opioids, Narcotic, Analgesia, Surgery, Breast, Anorectal 


\section{SUMMARY FOR LAY AUDIENCE}

Opioid overdoses have become one of the leading causes of preventable deaths in North America. Surgeons play a major role for many reasons. First, prescription opioids are still a major source of opioid misuse. Second, the risk of chronic opioid use after surgery is 5-15\%. Finally, diversion of excess, unused prescriptions is a major contributor to opioid misuse.

The impact of implementing a new standardized pain care bundle, termed "The STandardization of Outpatient Procedure Narcotics (STOP Narcotics)", was assessed with the objective of adequately controlling patient pain while reducing post-operative opioids after breast and anorectal outpatient surgery.

The multi-pronged intervention consisted of 4 main components: Patient Education was directed towards clarifying pain expectations, and coupled with written instructions for optimal utilization of medications for pain control; Provider Education focused on understanding the recommended multi-modal analgesic strategies; Intra-Operative Pain Management Strategy included medications targeting pain control and nausea were given by the anesthesiologist; and, Post-Operative Pain Management Strategy, where prescription for an anti-inflammatory was given, along with instructions for regular acetaminophen use. A separate optional prescription for a reduced amount of an opioid was also given.

Through clarifying patient expectations and providing clear instructions on how to use nonopioid medications effectively for pain, equivalent post-operative pain control was achieved, along with increased patient satisfaction in the post-intervention group. By using multiple nonopioid medications there was a 50\% reduction in opioid prescribing. More significantly, only $45 \%$ of patients filled their opioid prescription. There was no significant difference in prescription refills, and appropriate medication disposal of excess medication increased after the intervention was implemented.

If this intervention was implemented province-wide (over 20,000 breast procedures are performed in Ontario each year) and opioid prescriptions decreased from 30 pills to 10 pills with only $45 \%$ of patients filling their prescription, this would result in a decrease of pills consumed from 600,000 pills to 90,000 pills for breast surgery patients alone. Similar standardized interventions may be implemented at other institutions and expanded to other more complex procedures and surgical disciplines, and may provide a foundation for future guidelines and impact the opioid epidemic in a truly meaningful way. 


\section{COAUTHORSHIP:}

While each of the co-authors listed below made important contributions to this work, Luke B. Hartford is the lead author responsible for the background, methodology, data collection, analysis and publication of results. All manuscripts and chapters presented in this thesis were prepared by the author, with consultation and critical review by the co-authors.

Julie Ann M. Van Koughnett, MD, MEd, FRCSC, FACS - My research supervisor who provided guidance throughout my thesis. She was involved in the creation of the thesis structure, methodology, data interpretation and manuscript revisions. She scheduled regular meetings and interim reports throughout completion of my thesis.

Daryl K. Gray, MD, FRCSC, FACS; Ken A. Leslie, MD, MHPE, FRCSC; Muriel Brackstone, MD, MSc, PhD, FRCSC - These research co-supervisors provided feedback and direction on methodology, data interpretation and manuscript revisions. All were instrumental in the review and publication of each chapter.

Patrick B. Murphy, MD, MSc, MPH, FRCSC - A co-author who provided guidance on methodology, statistical analysis, manuscript revisions and dissemination.

Collin F. M. Clarke, MD, FRCPC - A co-author instrumental in the development of our study protocol, in addition to implementation among anesthetists.

Allison H. Maciver, MD, MSc, FRCSC - A co-author involved in development and implementation of the study protocol specifically in breast surgery. She provided data interpretation and manuscript revision for the breast surgery publication.

Richard J. Hilsden, MD, MBA, FRCSC - A co-author involved in development and implementation of the study protocol, as well as data collection.

Sarah A. Knowles, MD, MSc, FRCSC - A co-author involved in implementation of the study protocol in breast surgery and also provided feedback in manuscript revisions.

Kelly N. Vogt, MD, MSc, FRCSC - A co-author involved in methodology, statistical analysis and manuscript revisions.

Neil G. Parry, MD, FRCSC, FACS - A co-author involved in development and implementation of the study protocol.

Laura J. Allen, MSc - A co-author instrumental in data collection, statistical analysis and the use of statistical software.

Carlos Garcia-Ochoa, MD; Robin B. Wigen, BSc; Samuel D. Gray, BSc - Co-authors involved in data collection and manuscript revisions. 


\section{ACKNOWLEDGEMENTS:}

I am thankful to all the co-authors for the completion and success of this work. They have invested a great amount of time and work into this project, and have unequivocally supported my research endeavours, making this thesis possible.

I thank the staff and residents of the Division of General Surgery, Department of Anesthesia and Perioperative Medicine, pre-admission clinic staff, day surgery staff, post-anesthesia care unit staff and general surgery clinic staff at London Health Sciences Centre who demonstrated exceptional support of this project, facilitating its completion.

Laura Allen was instrumental in this work and deserves recognition for her assistance with statistical analysis and use of statistical software. Her positive attitude, availability and tremendous effort to ensure thorough data collection and interpretation truly played a major role in this work.

Dr. Julie Ann Van Koughnett deserves mention as supervisor and mentor, who met regularly from creation to completion of this thesis. She provided timelines, constructive feedback and poured a tremendous amount of effort in manuscript review. She leads through example, and is a role model I would hope to emulate.

Dr. Ken Leslie deserves recognition as co-supervisor and a mentor who has dedicated himself to the highest quality of patient care. As a well-respected leader, his influence was the foundation for practice change across the Division of General Surgery.

Dr. Collin Clarke, an anesthetist who specializes in pain research, was an invaluable member of our team who helped evaluate evidence regarding analgesic medications and pain measurement scales. He was influential throughout the entire project, and in particular was instrumental in spreading the intervention through the anesthesia department.

Thank you to Dr. Supriya Singh and the orthopedic research team, who published "A prospective, randomized controlled trial of the impact of written discharge instructions for postoperative opioids on patient pain satisfaction and on minimizing opioid risk exposure in orthopaedic surgery". This thought-provoking research motivated the Division of General Surgery to reassess our opioid prescribing practices. Her willingness to share her experience was greatly appreciated.

Thank you to my wife, Megan Fortino, who is an incredibly understanding, intelligent, kind, and supporting partner and friend. Thank you for showing nothing but support through all the stages of my residency and thesis. 
TABLE OF CONTENTS

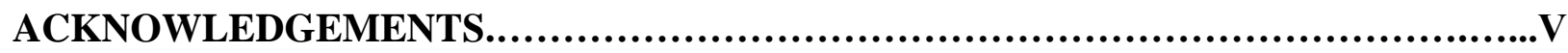

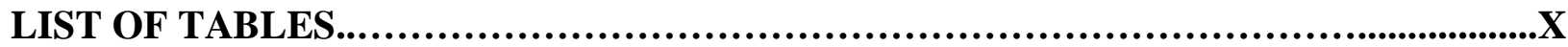

LIST OF FIGURES............................................................................

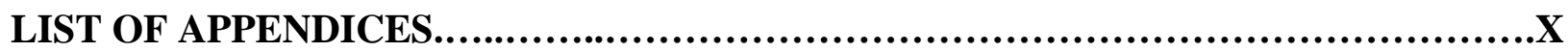

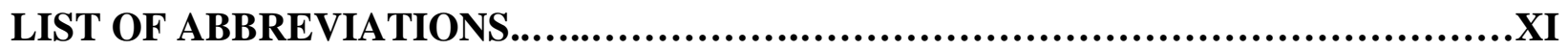

1.0 CHAPTER 1: INTRODUCTION AND REVIEW ..................................................................... 1

1.1 OPIOID RESEARCH IN SURGERY ............................................. 1

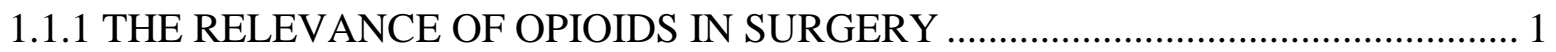

1.1.2 RESEARCH-BASED STANDARDIZED SCALE ................................................ 1

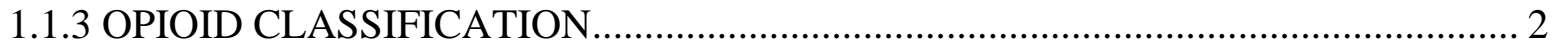

a. Natural, semi-synthetic and synthetic opioids ..................................................... 2

b. Opioid receptors and intrinsic activity ................................................................ 3

1.1.4 OPIOID MISUSE, ABUSE, ADDICTION, TOLERANCE, AND DEPENDENCE ... 3

1.1.5 PATHOPHYSIOLOGY OF OPIOID DEPENDENCE ........................................... 3

1.2 EPIDEMIOLOGY OF THE OPIOID CRISIS .................................................................... 4

1.2.1 EPIDEMIOLOGY OF GLOBAL DRUG ABUSE …….......................................... 4

1.2.2 EPIDEMIOLOGY OF OPIOID ABUSE IN NORTH AMERICA …….......................... 4

a. Epidemiology of opioid abuse in the United States .................................................... 4

b. Epidemiology of opioid abuse in Canada ……………......................................... 5

c. Epidemiology of opioid abuse in London, Ontario, Canada ……………………...... 5

1.2.3 CLASSESS OF OPIOIDS THAT ARE MISUSED …….............................................. 6

1.2.4 PRESCRIPTION TRENDS OF OPIOIDS IN NORTH AMERICA............................ 7

1.2.5 HISTORY OF THE PHARMACEUTICAL OPIOID CRISIS .................................. 7

1.3 THE ROLE OF THE SURGEON AND CARE TEAM ...................................................... 8

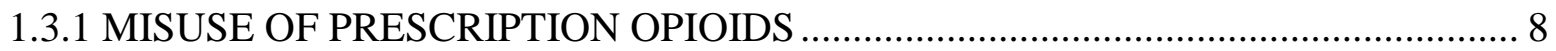

1.3.2 RISK OF CHRONIC OPIOID USE AFTER SURGERY ……….............................. 10

a. Risk factors for chronic opioid use ..................................................................... 12

1.3.3 VARIABLE AND EXCESSIVE OPIOID PRESCRIPTIONS ................................... 12

1.3.4 APPROPRIATE DISPOSAL OF OPIOIDS ......................................................... 14

1.4 THE IMPORTANCE OF ANALGESIA IN OUTPATIENT SURGERY ..................... 15

\subsection{INTERVENTIONS FOR OPIOID REDUCTION AND EFFECTIVE ANALGESIA}

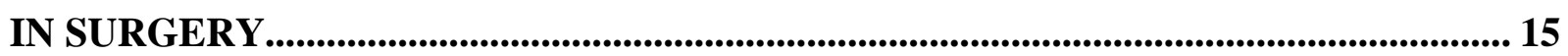

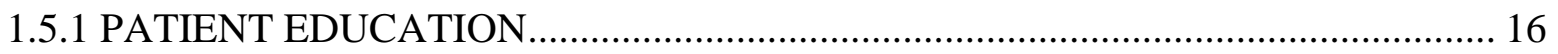

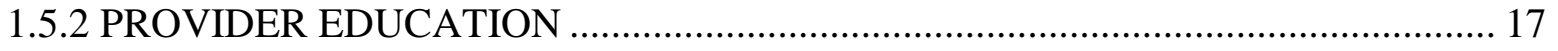

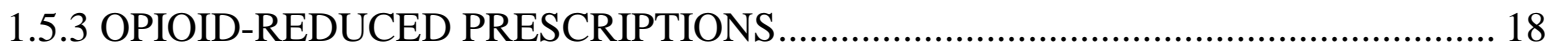

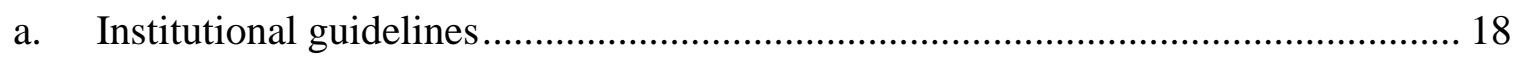

b. Electronic health systems ................................................................................. 18

1.5.4 NON-OPIOID MULTIMODAL ANALGESIC STRATEGIES. ................................ 18

1.5.5 PRE-EMPTIVE AND PRE-OPERATIVE MULTIMODAL ANALGESIA ................ 19 
a. Local Anesthetic Nerve Blocks. ........................................................................ 19

b. Regional Anesthetic Nerve Blocks.................................................................... 19

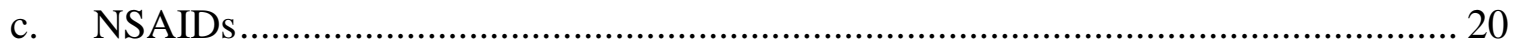

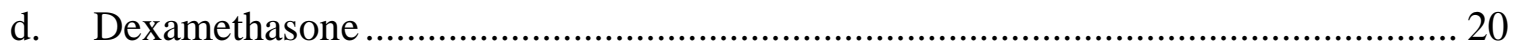

1.5.6 POST-OPERATIVE OPIOID-SPARING MULTIMODAL ANALGESIA ................ 21

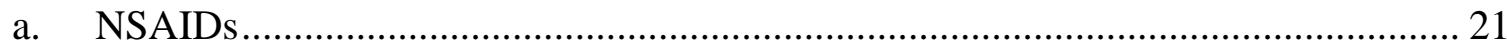

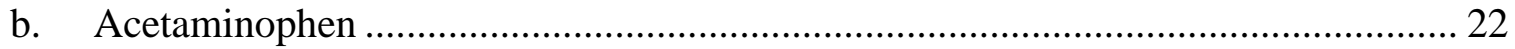

c. NMDA antagonists, Alpha-2 agonists, Gabapentinoids, Selective Serotonin

Reuptake Inhibitors and Tricyclic Antidepressants ..................................................... 22

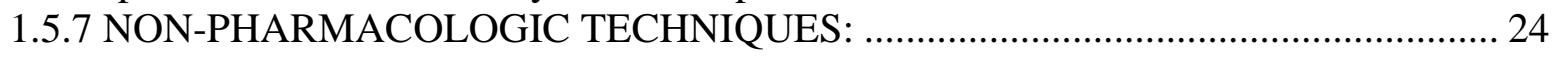

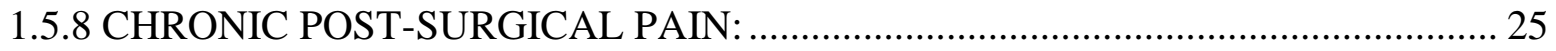

1.6 THE CONCEPT OF "BUNDLES” IN SURGERY ........................................................... 26

1.7 OBJECTIVES AND PURPOSE OF THE THESIS ..................................................... 26

1.7.1 DEFINING THE PROBLEM IN BREAST AND ANORECTAL SURGERY .......... 26

1.7.2 ORTHOPEDIC EDUCATIONAL RCT AT LHSC ……………............................... 27

1.7.3 DEVELOPMENT OF THE STOP NARCOTICS INITIATIVE .................................. 27

1.7.4 HISTORY OF HERNIA AND LAPAROSCOPIC CHOLECYSTECTOMY ............. 28

1.7.5 RETROSPECTIVE REVIEW OF PRESCRIBING PRACTICES …….................... 28

1.8 PURPOSE STATEMENT AND HYPOTHESES ...................................................... 31

1.9 REFERENCES................................................................................................................................ 32

2.0 CHAPTER 2. THE STANDARDIZATION OF OUTPATIENT PROCEDURE (STOP) NARCOTICS AFTER ANORECTAL SURGERY: A PROSPECTIVE NONINFERIORITY STUDY TO REDUCE OPIOID USE.......................................................... 47

2.1 BACKGROUND: ............................................................................................................................ 47

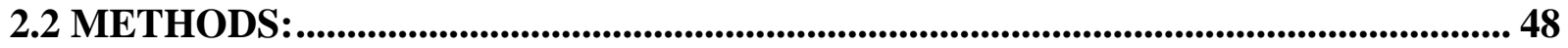

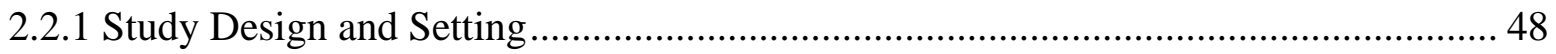

2.2.2 Pre- and Post-Intervention Groups........................................................................... 48

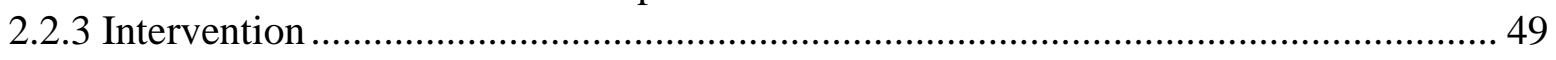

Patient Education.............................................................................................................. 49

Provider Education .................................................................................................. 50

Intra-Operative Pain Management Strategy .............................................................. 50

Post-Operative Pain Management Strategy .................................................................. 50

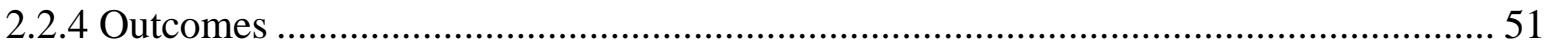

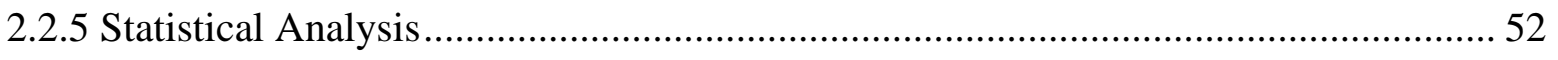

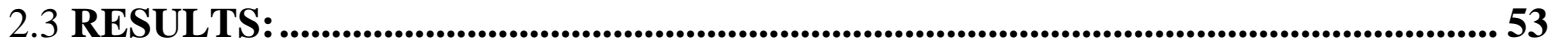

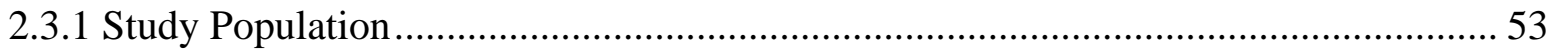

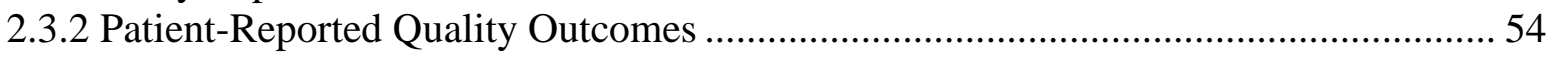

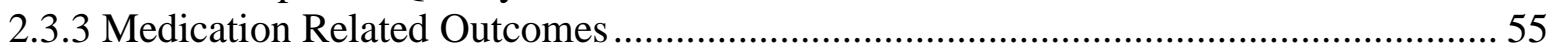

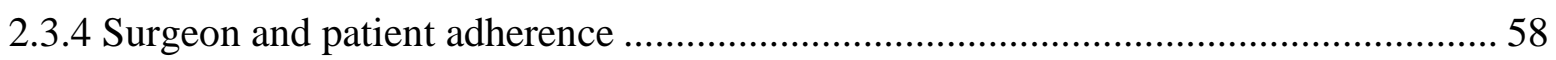

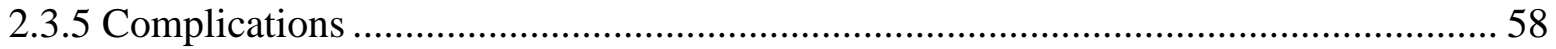


2.4 DISCUSSION:........................................................................................................................... 58

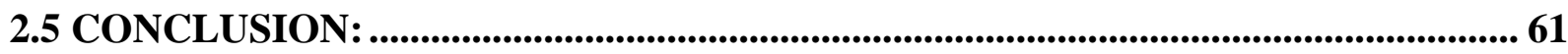

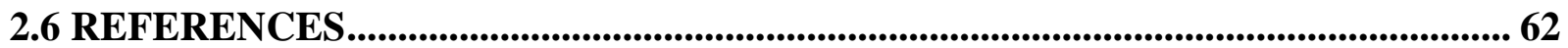

APPENDIX 5.2 Post-operative pain management strategy for anorectal surgery.......... 100

3.0 CHAPTER 3. THE STANDARDIZATION OF OUTPATIENT PROCEDURE (STOP) NARCOTICS: A PROSPECTIVE HEALTH SYSTEMS INTERVENTION TO REDUCE OPIOID USE IN AMBULATORY BREAST SURGERY ......................................................... 65

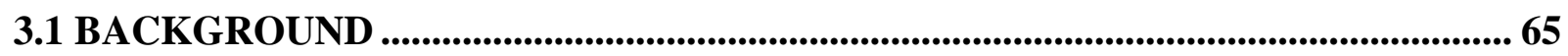

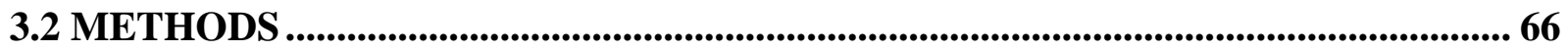

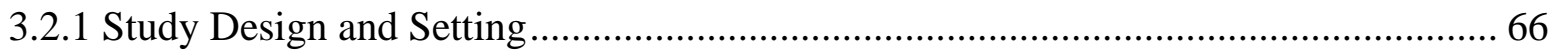

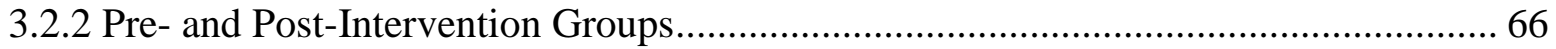

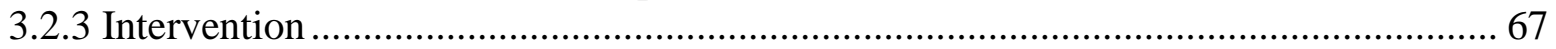

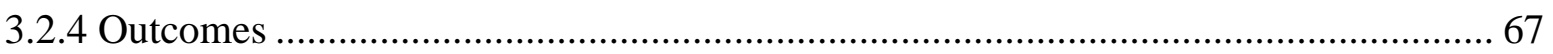

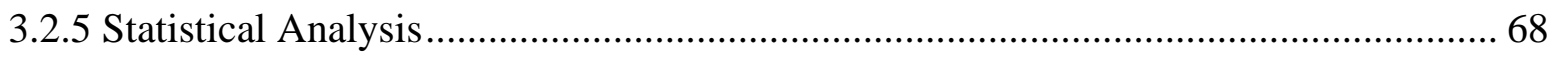

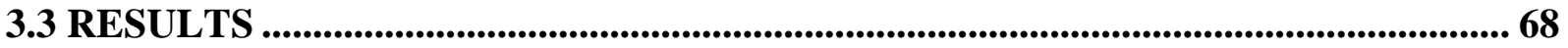

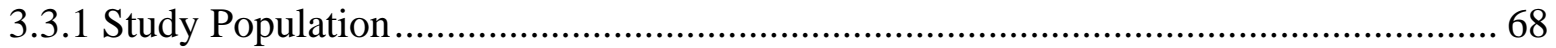

3.3.2 Primary Analgesia Outcome and Quality of Pain Control.......................................... 70

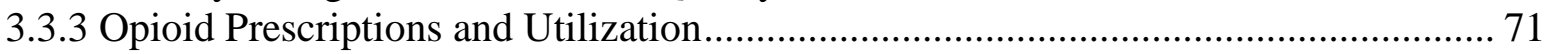

3.3.4 Analgesic Non-Opioid Adjuncts and Medication Disposal........................................ 72

3.3.5 Subgroup Population Analysis .......................................................................... 72

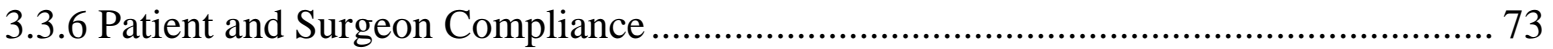

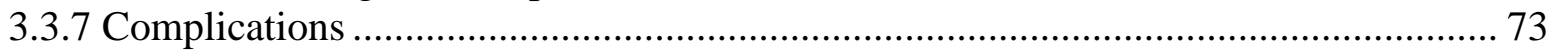

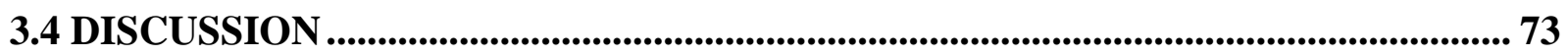

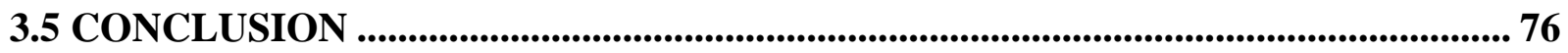

3.6 REFERENCES...................................................................................................................... 77

APPENDIX 5.3 Multi-pronged opioid-sparing intervention .................................................. 101

APPENDIX 5.4 Post-operative pain management instructions for breast surgery: ...... 102

4.0 CHAPTER 4 DISSCUSSION AND DISSEMINATION ...................................................... 83

4.1 SUMMARY OF MAJOR FINDINGS ............................................................................ 83

4.1.1 Patient-reported pain after surgery ........................................................................ 83

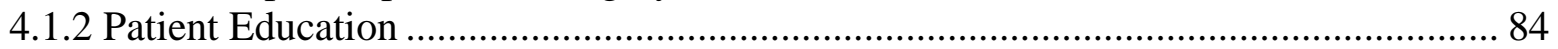

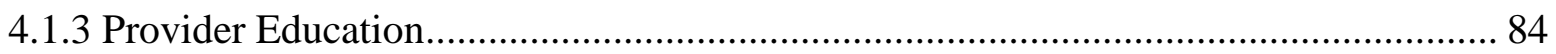

4.1.4 Intra- and Post-Operative Medication Utilization and Prescribing............................. 85

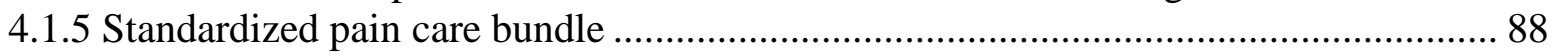

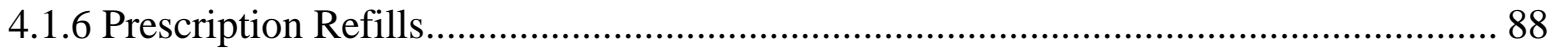

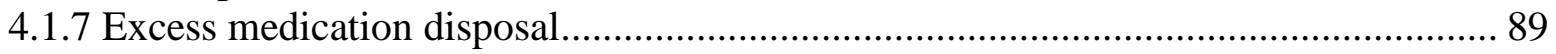

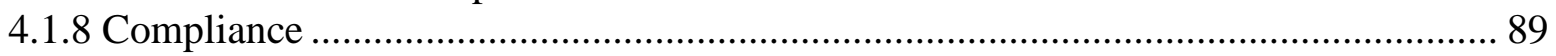




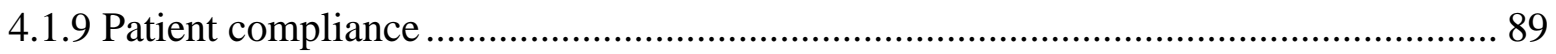

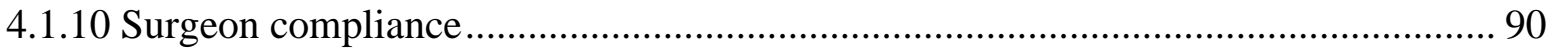

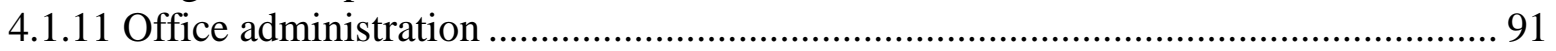

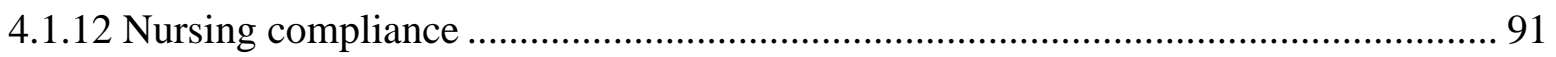

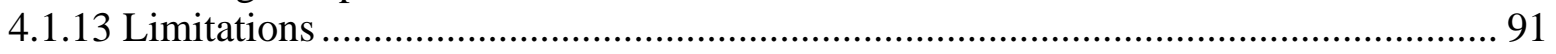

4.2 DISSEMINATION....................................................................................................................... 93

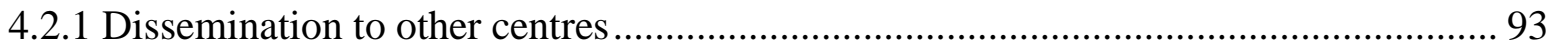

4.2.2 Dissemination to other surgical specialities ................................................... 95

4.2.3 Follow up and Study Dissemination .............................................................. 95

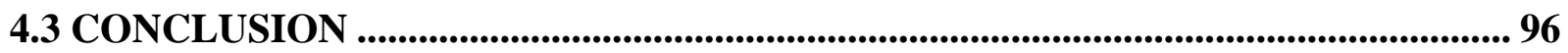

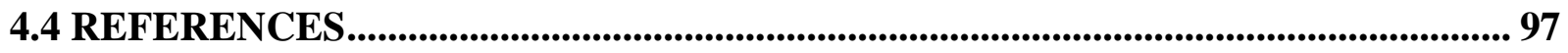

APPENDIX 5.5 THE STANDARDIZATION OF OUTPATIENT PROCEDURE NARCOTICS: A PROSPECTIVE NON-INFERIORITY STUDY TO REDUCE OPIOID USE IN OUTPATIENT GENERAL SURGICAL

PROCEDURES...........................................................................103

CURRICULUM VITAE...................................................................104 


\section{LIST OF TABLES:}

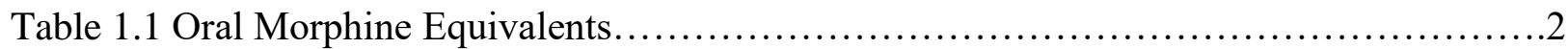

Table 1.2 Outcomes of outpatient hernia and laparoscopic cholecystectomy....................28

Table 2.1 Oral Morphine Equivalents...................................................

Table 2.2 Patient characteristics in anorectal surgery......................................54

Table 2.3 Pre- and post-intervention comparison of pain control............................55

Table 2.4 Pre- and post-intervention comparison of medications.............................56

Table 2.5 Pre- and post-intervention comparison: hemorrhoidectomy subgroup................57

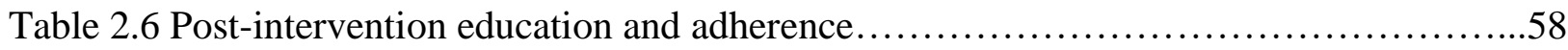

Table 3.1 Patient characteristics in breast surgery ........................................69

Table 3.2 Pre- and post-intervention comparison of pain, quality and function.................70

Table 3.3 Pre- and post-intervention comparison of medications ............................71

Table 3.4 Medication comparison in PM/SM, PM/SM + SLN, and ALND/MRM...............73

\section{LIST OF FIGURES:}

FIGURE 1.1 Opioid overdose deaths by type, United States $2007-2017 \ldots \ldots \ldots \ldots \ldots \ldots \ldots \ldots \ldots \ldots$

FIGURE 1.2 Sources of misused prescription opioids among people aged 12 or older in $2016 \ldots 9$

FIGURE 1.3 Retrospective data of median opioid pills prescribed in outpatient breast and anorectal surgeries over 3 months at LHSC and SJHC ....................................

FIGURE 1.4 Opioid prescriptions by type in 145 breast surgery patients..................... 30

FIGURE 1.5 Opioid prescriptions by type in 56 anorectal surgery patients....................30

FIGURE 2.1 Flow chart of included patients anorectal surgery ............................53

FIGURE 2.2 Utilization of multimodal analgesia medications, pre- and post-intervention.......57

FIGURE 3.1 Flow chart of included patients breast surgery ..............................69 


\section{LIST OF APPENDICES:}

APPENDIX 5.1 Research ethics board approval form..................................99

APPENDIX 5.2 Post-operative pain management strategy anorectal surgery ..................100

APPENDIX 5.3 Post-operative pain management strategy breast surgery $\ldots \ldots \ldots \ldots \ldots \ldots \ldots \ldots 101$

APPENDIX 5.4 Multi-pronged, opioid sparing intervention for breast surgery ..............102

APPENDIX 5.5 STOP in outpatient hernia and laparoscopic cholecystectomy...............103

\section{LIST OF ABBREVIATIONS}

STOP Narcotics - Standardization of Outpatient Procedure Narcotics

OME - Oral Morphine Equivalents

WHO - World Health Organization

US - United States

CIHI - Canadian Institute for Health Information

PM - partial mastectomy

SM - simple mastectomy

SLN - sentinel lymph node

ALND - axillary lymph node dissection

MRM - modified radical mastectomy

LC - laparoscopic cholecystectomy

IH - inguinal hernia

UH - umbilical hernia

EUA - examination under anesthesia

EMR - electronic medical records

$\mathrm{RCT}$ - randomized control trial

PAC - preadmission clinic

PACU - post-anesthetic care unit 


\subsection{CHAPTER 1: INTRODUCTION AND REVIEW}

\subsection{OPIOID RESEARCH IN SURGERY}

\subsubsection{THE RELEVANCE OF OPIOIDS IN SURGERY}

It is currently common practice for patients undergoing surgeries to receive an opioid prescription for pain control as part of their post-operative care. While effective analgesic agents, these psychoactive medications derived from opium or synthetically derived analogues also carry the potential for misuse secondary to their euphoric effects. ${ }^{1,2}$ Common examples used in surgery include morphine, codeine, hydromorphone, oxycodone, hydrocodone, fentanyl, and tramadol. ${ }^{2}$ With the dramatic rise in opioid medication abuse over the past decade, research in opioid prescribing after surgery is a timely and relevant subject.

\subsubsection{RESEARCH-BASED STANDARDIZED SCALE}

Due to the different potencies among opioids, the number of pills consumed is an inaccurate method of measuring and comparing opioid quantities. The accepted research-based method of measurement is a standardized scale, comparing different opioid drugs to the equivalent of 1 milligram of oral morphine (Table 1.1$){ }^{3}$ This is referred to as oral morphine equivalents (OME) and takes into account the differences in opioid drug type and strength. ${ }^{3}$ 
Table 1.1 Oral Morphine Equivalents (OME)

\begin{tabular}{lc}
\hline \multicolumn{1}{c}{ Oral Morphine Equivalents } \\
\\
$\qquad \begin{array}{l}\text { 10 tablets } \\
\end{array}$ \\
\hline Morphine $5 \mathrm{mg}$ & OME \\
\hline Oxycodone $5 \mathrm{mg}$ & 50 \\
\hline Codeine $30 \mathrm{mg}$ & 75 \\
\hline Tramadol $50 \mathrm{mg}$ & 45 \\
\hline Hydromorphone 2 mg & 50 \\
\hline
\end{tabular}

Oral Morphine Equivalents for 10 tablets of common surgical prescriptions.

Agency Medical Directors' Group. ${ }^{27}$ http://www.agencymeddirectors.wa.gov/calculator/dosecalculator.htm

\subsubsection{OPIOID CLASSIFICATION}

Opioids may be classified according to their chemistry, receptor, or intrinsic activity. ${ }^{4}$

\section{a. Natural, semi-synthetic and synthetic opioids}

"Natural" opioids, also termed "alkaloid" opioids, are composed of "natural" compounds, with familiar examples including codeine and morphine. ${ }^{1,4}$ When a natural opioid's structure is chemically manipulated, "semi-synthetic" opioids are derived which include heroin, hydromorphone, oxycodone and hydrocodone. ${ }^{1,4}$ Common "synthetic" opioids include tramadol, methadone, fentanyl and carfentanil, which are manufactured in a laboratory. These have a chemical structure similar to opioids to produce opiate-like effects. ${ }^{1,4}$ Patterns may be traced over the past 30 years that highlight the misuse of each of these opioid classes during distinct time periods (See classes of opioids misused, page 3). 


\section{b. Opioid receptors and intrinsic activity}

Opioids are characterized by their complex interactions between three opioids receptors. ${ }^{1,4}$ Previously known as mu, delta, and kappa these are now commonly termed MOP (mu opioid), DOP (delta opioid), and KOP (kappa opioid). ${ }^{4}$ Opioids may act at these receptors as agonists, antagonists, and partial agonists. ${ }^{1,4}$ Opioids are effective analgesics, with multiple mechanisms and pathways contributing to their analgesic effects. The most relevant of these is their agonistic action on MOP receptors in the peripheral and central nervous systems. ${ }^{1,4}$

\subsubsection{OPIOID MISUSE, ABUSE, ADDICTION, TOLERANCE, AND DEPENDENCE}

Prescription opioid misuse is defined as using the medication for purposes other than the reason prescribed. ${ }^{5}$ Abuse is defined as consuming the opioid for euphoric effects, rather than for pain. ${ }^{5}$ Addiction is the continued seeking out of the drug, despite negative consequences on personal life, such as family, work, and friends. ${ }^{5}$ Tolerance is the escalation of consuming higher doses of the opioid, to achieve the same effects in relieving pain or obtaining euphoria. ${ }^{5}$ Physical dependence is established if withdrawal symptoms occur when the opioid is discontinued. ${ }^{5}$

\subsubsection{PATHOPHYSIOLOGY OF OPIOID DEPENDENCE}

Consuming opioids leads to an exposure risk for chronic use and subsequently misuse disorders. This is particularly true when a more potent or higher dosage of opioid is used, or if the use is frequent or for an extended duration. ${ }^{6,7}$ In addition to analgesia, through neurochemical pathways opioids also produce euphoria. This euphoria is central to the risk of prescription drug misuse. ${ }^{6}$ With repeated exposure to opioids, higher doses are required to replicate both euphoric and analgesic effects, leading to opioid tolerance. ${ }^{5,6}$ Withdrawal occurs when opioid suppression of noradrenaline ceases, resulting in distressing symptoms such as anxiety, agitation, insomnia, sweating, muscle cramps and diarrhea. ${ }^{6}$ Once dependence is established, addiction occurs when there is impaired judgement over use and the desire to obtain the medication exceeds other priorities resulting in harmful consequences. ${ }^{6,7}$ 


\subsection{EPIDEMIOLOGY OF THE OPIOID CRISIS}

\subsubsection{EPIDEMIOLOGY OF GLOBAL DRUG ABUSE}

Globally, drug abuse has risen to epidemic proportions. In 2016, illegitimate drug use was reported in 5.6\% of the global population (approximately 275 million people per year). ${ }^{8}$ In 2015 , the World Health Organization (WHO) estimated 450,000 deaths as a result of drug use, with $76 \%$ of these deaths attributed to opioid use disorders and overdose. ${ }^{8,9}$ Initially promoted as one of the most powerful analgesic agents, opioids are now one of the leading causes of injuryrelated deaths, and cause more harm than any other drugs worldwide. ${ }^{8,9}$

\subsubsection{EPIDEMIOLOGY OF OPIOID ABUSE IN NORTH AMERICA}

North America accounts for $85 \%$ of global opioid consumption. ${ }^{10}$ The United States (US) and Canada are the first and second largest consumers of prescription opioids per capita in the world, respectively. ${ }^{10}$

The statistics of deaths due to opioid overdoses are alarming. However, for every opioid overdose there are many more non-fatal overdoses. ${ }^{8,11}$ The resultant utilization of emergency medical calls and transportation, emergency department visits, hospitalizations and intensive care unit stays have had profound economic and emotional effects on patients, families, society and health care systems. ${ }^{11-15}$

\section{a. Epidemiology of opioid abuse in the United States}

In the United States, opioid-related morbidity and mortality continues to rise, with the mortality rate from opioid overdoses increasing six-fold from 1999 to $2017 .{ }^{11,16}$ During this time interval, there were almost 400,000 Americans deaths, with an average of 130 deaths per day in $2017 .{ }^{11,16}$ Deaths from opioid overdoses exceeded deaths attributed to motor vehicle collisions in $2016 .{ }^{15}$ The death rate in 2017 was 21.7 per 100,000 (47,700 overdose deaths), with 28,000 of these due 
to synthetic opioids such as fentanyl. ${ }^{11}$ The remainder of the overdose deaths are due to prescription opioids and heroin. ${ }^{11}$

\section{b. Epidemiology of opioid abuse in Canada}

In Canada, opioid-related morbidity and mortality parallels that of the United States. Deaths due to opioid overdoses increased $463 \%$ from the year 2000 to $2013 .{ }^{14}$ In 2016, there were over 2,800 opioid-related deaths (rate of 7.8 deaths per 100,000), or an average of 8 Canadians per day. ${ }^{14,17}$ Indeed, this was again greater than the average number of Canadians killed daily in motor vehicle collisions in $2015 .{ }^{17}$ Fentanyl-related opioids were responsible for $46 \%$ of these deaths, with prescription opioids and heroin responsible for the remainder. ${ }^{14}$

\section{c. Epidemiology of opioid abuse in London, Ontario, Canada}

The province of Ontario leads Canada in opioid-related deaths. ${ }^{18}$ In 2017, there were over 3 times the number of deaths from opioids overdoses $(1,250)$ compared to motor vehicle collision related fatalities (340). ${ }^{19}$ Despite this, prescriptions for opioids are still widespread, with $14 \%$ of the population filling an opioid prescription in 2015 (an estimated 1 in 7 people), totalling more than 9 million prescriptions. ${ }^{20}$

It is encouraging that the number of individuals filling opioid prescriptions in Ontario has remained stable, with no major increase over the past few years. ${ }^{20}$ However, more potent opioids are being prescribed more frequently. ${ }^{20}$ Hydromorphone prescriptions, for example, increased by $29 \%$ between 2013 to $2016 .{ }^{20}$

In Middlesex-London, Ontario, Canada where this integrated thesis work completed, mortality related to opioid overdoses was twice as high as the rest of the Ontario in 2012. ${ }^{21}$ Opioid-related emergency department visits, hospitalization rates, and admission to substance misuse programs consistently exceeded Ontario's average rates. ${ }^{21}$ 


\subsubsection{CLASSES OF OPIOIDS THAT ARE MISUSED}

Since the 1990s, different opiate classes have been implicated in opioid overdoses during three separate time intervals. An increase in overdoses from 1999-2010 was likely due to a rapid increase in physicians prescribing opioids in the 1990s, and overdose deaths involved natural (e.g. morphine, codeine) and semi-synthetic (e.g. hydromorphone, oxycodone, hydrocodone) opioids. ${ }^{11,22,23}$ The second rise was a sudden increase in overdose deaths in 2010 due to community dissemination of heroin. ${ }^{11,22}$ Then in 2013, there was a significant increase in deaths due to synthetic opioids (such as fentanyl and carfentanil), particularly illicitly manufactured fentanyl. ${ }^{11,15,22}$ Prescription opioids, heroin and synthetic illicit opioids all still play major roles in opioid morbidity and mortality today.

Figure 1.1

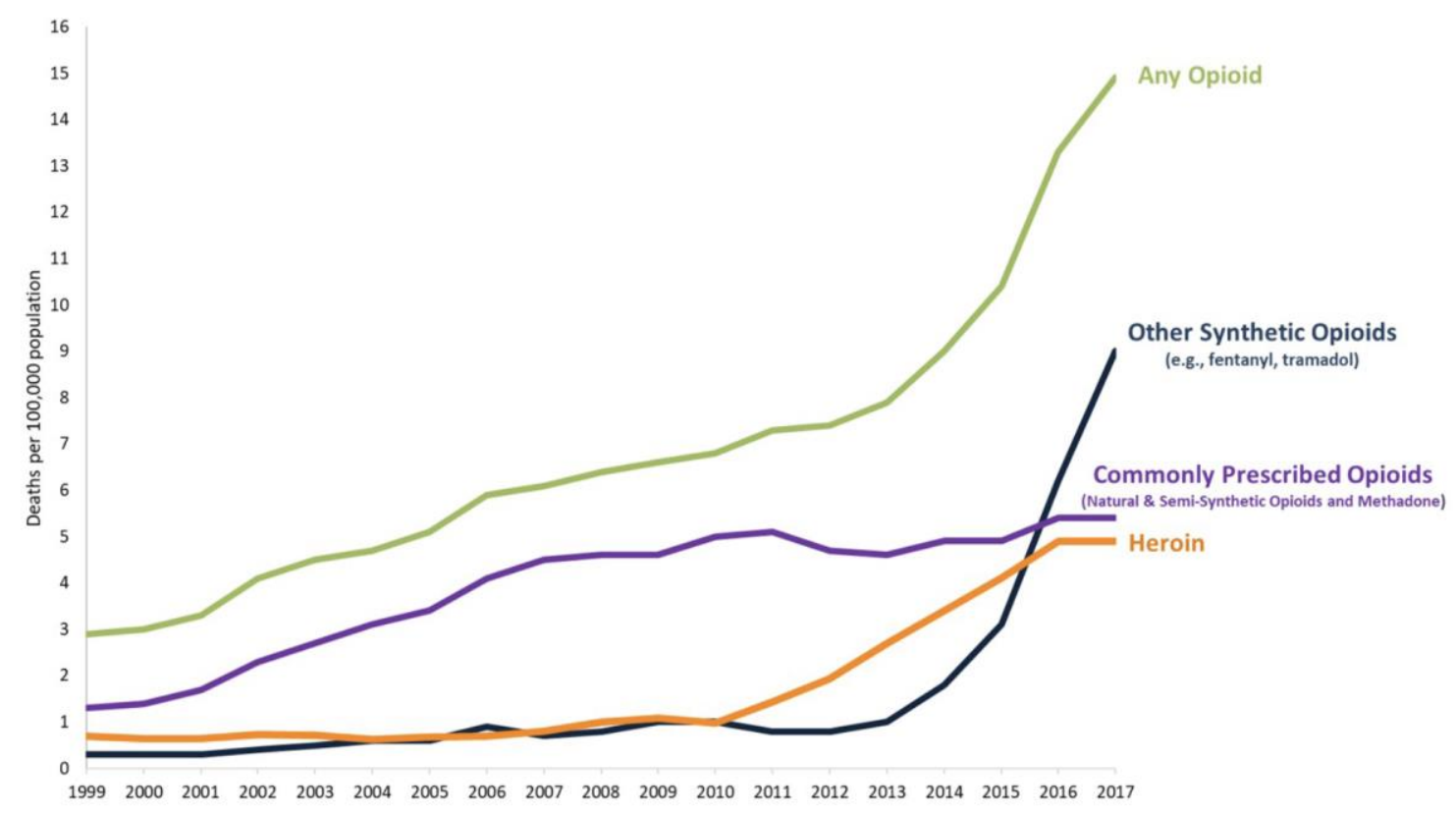

Figure 1.1 Overdose Deaths by Type, United States, 2007-2017; National Vital Statistics System, Centers for Disease Control (CDC), available at:

https://www.cdc.gov/drugoverdose/data/analysis.html ${ }^{24}$ 


\subsubsection{PRESCRIPTION TRENDS OF OPIOIDS IN NORTH AMERICA}

While the number of opioid prescriptions remains high, the overall opioid prescribing rate has plateaued and is decreasing in some locations. This suggests that health care providers may have become more cognitive about the risks of opioid prescriptions and are more cautious in their prescribing practices.

In the United States the opioid prescribing rate has declined 19\% from 2006 to $2017 .{ }^{11}$ Despite this, the amount of opioids prescribed per person still remains 3 times higher than previously reported in 1999. ${ }^{11}$ In 2017 there were still more than 17\% of Americans that filled at least one opioid prescription that year. ${ }^{11}$

In Canada, though the overall quantity of opioid (oral morphine equivalents) in each prescription decreased by 5\%, the number of opioid prescriptions continued to rise by $7 \%$ between 2012 and $2016 .{ }^{25}$ Opioid prescriptions were used by $13 \%$ of the population in 2017 , a decrease from $15 \%$ in 2013. ${ }^{14}$ This may reflect smaller but more frequent prescriptions to fewer individuals. Of this $13 \%$, approximately $2 \%$ of the population reported using the prescription for non-medical purposes. ${ }^{14}$ The number of fentanyl prescriptions decreased by $7 \%$, but the proportion of prescriptions for stronger opioids (for example, hydromorphone) increased by 10\% from 2012 and $2016 .^{25}$

\subsubsection{HISTORY OF THE PHARMACEUTICAL OPIOID CRISIS}

While illegal sources and production of opiates has increased, a large rise in morbidity and mortality has actually been associated with increased use of legitimately obtained pharmaceutical prescription opioids. ${ }^{8,9,22,23}$

This initial rise began in the 1990s when pain specialists and advocacy organizations began promoting pain as the "fifth vital sign", and that our nation was facing an epidemic of untreated pain. ${ }^{7,22,23}$ Industry-sponsored pain management training events and conferences, sophisticated 
marketing strategies and the promotion of opioid use for non-cancer pain all influenced physician prescribing practices. ${ }^{23}$

During this time period, the number of opioid prescriptions increased dramatically. Written opioid prescriptions increased by 300\% in the United States between 1991 and 2009. ${ }^{13}$ In Canada, written oxycodone prescriptions increased by $850 \%$ between 1991 and $2007 .{ }^{26}$

While there was comparable efficacy to other opioids, with the aggressive marketing of oxycontin, global sales rose from US \$48 million in 1996 to $\$ 2.4$ billion in 2012. ${ }^{7,22,23}$ This increase was paralleled to a lesser extent for other opioids, which also experienced an unprecedented rise. , $22,23^{2}$

Opioid misuse, addiction and overdose deaths have paralleled the volume of opioids prescribed. ${ }^{13}$ This public health crisis has now been recognized and while primary, secondary and tertiary prevention are all areas of focus there is no simple solution. North America is currently dealing with the aftermath of this epidemic, the impact of which will affect individuals, families, society, and health care for decades to come.

\subsection{THE ROLE OF THE SURGEON AND CARE TEAM}

With the recent spike in opioid overdoses secondary to illicit fentanyl, and also the attention surrounding heroin misuse, it may seem that surgeons play a minimal role in the opioid crisis, as those medications are not routinely prescribed. In addition, much responsibility has been attributed to opioids prescribed for chronic non-cancer pain (not commonly managed by surgeons), leading to chronic use and eventual misuse.

The surgeon should assume an important role in the opioid crisis for multiple reasons. First, prescription opioids obtained from physicians, including surgeons still have a major role in opioid morbidity and mortality. Second, with every post-operative opioid prescription there is risk of chronic use and future opioid misuse disorder. Third, prescriptions after surgery are often 
excessive and variable, with only a fraction of the medication prescribed being consumed. Finally, appropriate disposal of excess medication is generally quite poor.

\subsubsection{MISUSE OF PRESCRIPTION OPIOIDS}

Misuse use of opioids is defined as consumption for purposes other than the reason prescribed. This includes use by individuals who obtained prescription opioids from other sources. ${ }^{14}$

Data from the National Survey on Drug Use and Health indicates that prescription opioid pain relievers for non-medical use are most commonly obtained from a friend or relative in the majority $(53.0 \%)$ of cases. ${ }^{27}$ Other sources included were from a single physician, "double doctoring" or prescriptions from multiple physicians, prescription fraud or forgery, theft, street drug dealers and internet purchases (Figure 1.2). ${ }^{14,27-29}$

Figure 1.2

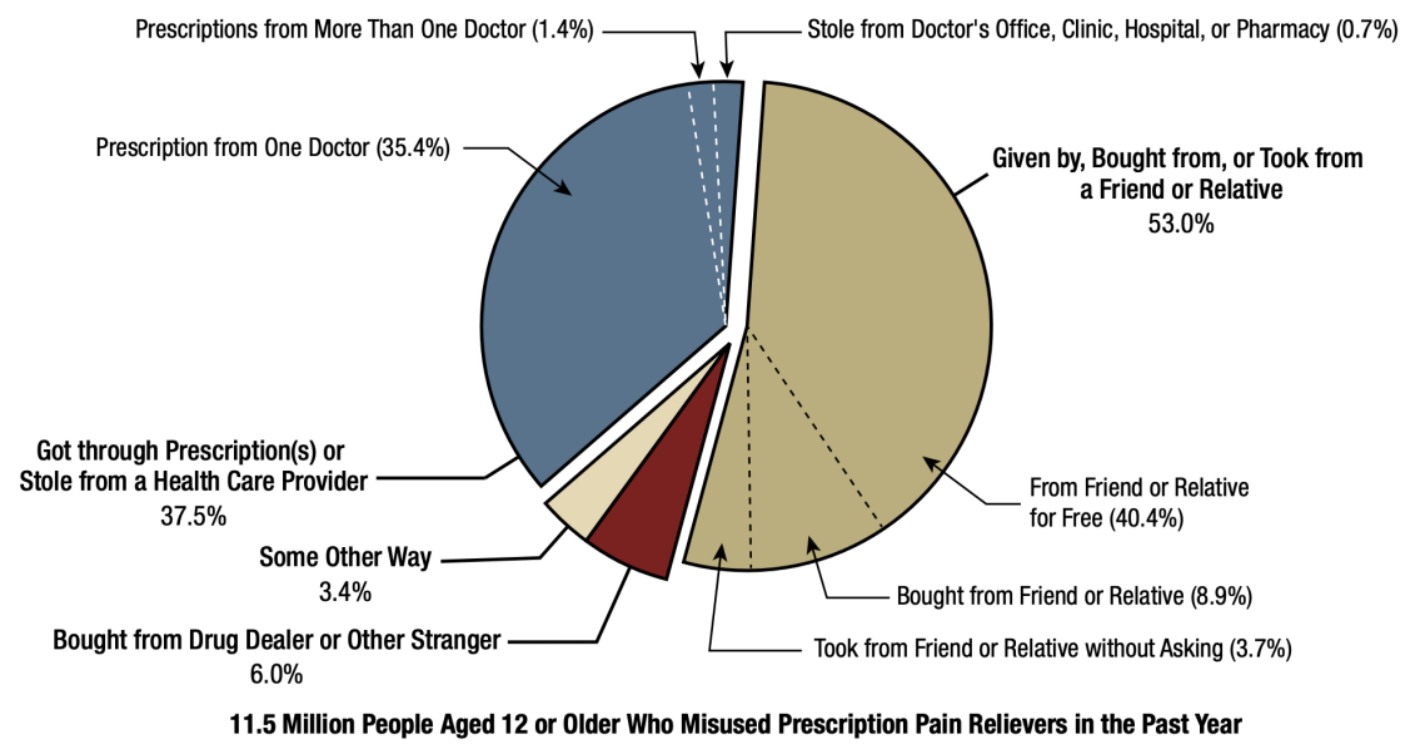

Figure 1.2 Sources of misused prescription opioids among people aged 12 or older in $2016 .{ }^{27}$ Available at: https://www.samhsa.gov/data/sites/default/files/NSDUH-FFR1-2016/NSDUHFFR1-2016.pdf 
The range of past year prescription opioid misuse prevalence in individuals aged 11-30 years was $0.7 \%-16.3 \% .^{30}$ The majority of non-medical users are men $(56 \%)$, between the ages of $18-34$

years $(54 \%)$, of a white race $(73.5 \%)$, with a low family income (less than US\$50,000). ${ }^{28}$

Fentanyl is unique in that it can be obtained both through the pharmaceutical industry, or illicitly manufactured. Therefore, it is difficult to ascertain the amount that is obtained legitimately through physicians and misused as a "prescription opioid".

Despite a plateau and decline of prescribing rates per person, prescription opioids remain a major cause of opioid morbidity and mortality in North America. The CDC reported prescription opioids accounted for 218,000 of the 400,000 deaths from opioid overdoses between 1999 and 2017. ${ }^{11,16}$

Currently, prescription opioids are involved in more than $35 \%$ of all overdose deaths in the United States alone. ${ }^{11,14}$ In addition, the majority of illicit fentanyl and nearly $75 \%$ of recent heroin users report being introduced to opioids through prescription medications. ${ }^{31}$

\subsubsection{RISK OF CHRONIC OPIOID USE AFTER SURGERY}

A prescription opioid may be a common avenue to opioid misuse disorders. ${ }^{31}$ Indeed, up to $75 \%$ of heroin users report being initially prescribed an opioid by a physician. ${ }^{31}$ Surgery may also be a risk factor for chronic opioid use. In a survey of chronic pain patients, one-third reported receiving their initial opioid prescription from a surgeon. ${ }^{32}$

In a study of 536,767 opioid naïve patients who filled an opioid prescription, $5 \%$ became long term users. ${ }^{33}$ Brummet retrospectively analyzed 36,177 surgical patients undergoing both major and minor surgery. ${ }^{34}$ Major procedures included ventral incisional hernia repair, colectomy, reflux surgery, bariatric surgery, and hysterectomy. Minor procedures included varicose vein removal, laparoscopic cholecystectomy, laparoscopic appendectomy, hemorrhoidectomy, thyroidectomy, transurethral resection of the prostate, parathyroidectomy and carpal tunnel 
release. New persistent opioid use between 90 and 180 days was increased to a similar extent in both groups (major surgery $6.5 \%$ vs minor surgery $5.9 \%$ ). ${ }^{34}$

When defining chronic opioid use as a prescription for an opioid between 60 days to 1 year after surgery, Alam demonstrated that patients were $44 \%$ more likely to develop chronic use compared to those who did not receive an opioid. ${ }^{35}$ When examining 39,140 of opioid naïve patients 66 years of age or older, Clarke demonstrated $3.1 \%$ of patients continued to receive opioids for more than 90 days after surgery. ${ }^{36}$ Sun described a lower incidence of $0.119 \%-1.41 \%$ prolonged opioid use after surgery in opioid naïve patients. ${ }^{37}$ This study may underestimate incidence as it defined chronic opioid use as more than 10 opioid prescriptions filled, or more than 120 days supplied between 3 and 12 months after surgery. ${ }^{37}$

In opioid naïve women after breast surgery $10 \%$ continued to fill prescriptions beyond 3 months after surgery. ${ }^{38}$ Anxiety and depression were identified as risk factors for using higher amounts of OME over prolonged periods of time, compared to those women without these variables $(13.4 \%$ vs $9.1 \%) .{ }^{38}$

Opioid-naïve cancer patients are at increased risk for chronic opioid use defined as opioid prescriptions 90-180 days after surgery. Lee evaluated 68,463 patients, and among opioid-naïve patients risk of persistent opioid use was $10.4 \% .{ }^{3940}$ This is particularly relevant, considering the recent trend to favourable prognosis and long-term survivorship of breast and colon cancers.

These studies re-emphasize the role of the surgeon through characterizing the opioid-exposure. With an increasing number of ambulatory surgeries in North America, a 5-15\% risk of chronic use comprises a substantial concern. ${ }^{41,42}$ While surgery is a risk factor for chronic use, the percentage of patients with chronic use that transition to misuse is unknown. However, once a patient transitions to chronic opioid use cessation is rare, and it is reasonable to postulate these patients may be at higher risk of eventual misuse. ${ }^{43,38}$ Therefore, if opioids are prescribed it emphasizes the need to intervene and taper in the immediate post-operative period. ${ }^{43,38}$ 


\section{a. Risk factors for chronic opioid use}

Surprisingly, intensity of pain after surgery may be unrelated to the risk of chronic opioid use. Indeed, in the study by Goesling including patients undergoing total hip and knee arthroplasty, the pain intensity in the $4-8 \%$ of patients with chronic use at 6 months was unrelated. ${ }^{34,44}$ Brummett et al compared 29,068 patients receiving minor surgical procedures to 7,109 major procedures. ${ }^{34}$ The rate of chronic opioid use was similar between the 2 groups ranging from $5.9 \%$ to $6.5 \% .^{34}$

Risk factors for new persistent opioid use include tobacco use, alcohol and substance use disorders, younger age, lower household income and comorbid conditions, regardless of surgical procedure completed. ${ }^{34}$ Other preoperative pain disorders (back pain, arthritis, and centralized pain conditions - specifically fibromyalgia), anxiety and depression have all been independently

associated with persistent opioid use. ${ }^{34,36,45}$ In patients who are undergoing curative-intent cancer surgery, chemotherapy was associated with a higher risk of chronic opioid use ${ }^{39}$ Chemotherapy and risk for peripheral neuropathy may account for this association. Specifically risk factors identified in outpatient procedures included age, depression, psychotropic medications, pain impairment and previous substance abuse. ${ }^{46}$

\subsubsection{VARIABLE AND EXCESSIVE OPIOID PRESCRIPTIONS}

Approximately 50 million outpatient procedures were performed in the United States in 2010. ${ }^{41,42}$ In Canada, the Canadian Institute for Health information (CIHI) reported 1.8 million outpatient procedures in $2005 .{ }^{47}$ The rates of outpatient surgical procedures increased by $300 \%$ in the 10-year period from 1996-2006 in the United States, and continues to increase in North America on an annual basis. ${ }^{41,42}$ Considering a significant portion of these patients receive an opioid prescription after outpatient surgery, this truly is an astounding amount of medication.

There is a lack of evidence-based guidelines that specifically address the appropriate quantity of opioid to prescribe after outpatient surgical procedures. Available clinical standards are available, however, are quite general and directed towards inpatient procedures, patient- 
controlled analgesia, and regional or multimodal techniques. ${ }^{48}$ Current surgical training curriculum provides inadequate training on appropriate post-operative analgesia for medical students and residents, leading to significant regional variation in prescribing practices.

The lack of guidelines has also contributed to excess opioid prescribing after surgery. Recently, studies have demonstrated that patients often consume only a fraction of the opioids prescribed..$^{49,50,51}$

Hill reported excessive and variable prescriptions among opioid-naïve patients. ${ }^{49}$ The median number of pills and range prescribed were as follows: partial mastectomy (PM) 20 (0-50); PM with sentinel lymph node biopsy (SLNB) 20 (0-60); laparoscopic cholecystectomy (LC) 30 (0100); open inguinal hernia (IH) $30(15-120) .{ }^{49}$ Overall, only $28 \%$ of the pills were actually consumed (PM 15\%, PM SLNB 25\%, LC 33\%, and IH 30\%), with less than 2\% of patients obtaining refills. ${ }^{49}$ In a variety of major inpatient surgical procedures, they reported patients consuming $38 \%$ of prescribed opioid pills, with 15 opioid pills satisfying the opioid needs of $88 \%$ of patients discharged on post-operative day one. ${ }^{52}$

Tan prospectively documented that opioid-naïve patients undergoing open and laparoscopic abdominal procedures were prescribed a median of 150 OME (equivalent to 20 oxycodone pills), but at their first post-operative visit had only consumed 30 OME or 4 pills. ${ }^{53}$ Only $4.5 \%$ of patients required additional prescriptions and $77 \%$ of patients were satisfied with their pain management. ${ }^{53}$ Fuji re-affirmed a wide range in the amount of opioid prescribed, of which only $27 \%$ was consumed after general and orthopedic surgical procedures. ${ }^{54}$

Subsequent studies have reproduced similar results and are summarized in two recent systematic reviews. ${ }^{51,50}$ Overall, the low quantity of opioid consumed may be underestimated considering they do not usually include if the patient received instructions for non-opioid multimodal analgesia, which may have further reduced opioids consumption.

While patients may only consume a fraction of the opioid prescribed, consumption does correlate and increase with higher opioid prescriptions. Howard demonstrated that for every additional pill 
prescribed, the patient will consume an additional one-half of a pill, likely due to an affect heuristic. ${ }^{55}$

These excessive prescriptions lead to unused medication which is one of the root problems in the non-medical use of prescription opioids. Considering data from the National Survey on Drug Use and Health indicates that prescription opioid pain relievers for non-medical use are most commonly obtained from a friend or relative in the majority of cases, this indeed poses a significant problem. ${ }^{27}$

\subsubsection{APPROPRIATE DISPOSAL OF OPIOIDS}

With the described findings of excess medication, a potential solution is appropriate disposal of this medication. Unfortunately, appropriate disposal at a pharmacy - even with specific instructions - is low (5-20\%).

Appropriate disposal methods described have included return to pharmacy, drug take-back programs, disposal in the trash after combining with an unpalatable substance or flushing down the toilet when options are not accessible. ${ }^{56}$ Medication disposal in the trash or flushing down the toilet has been discouraged due to environmental concerns.

Reports of appropriate disposal without instructions are approximately 5-10\%. Even with giving direct written disposal instructions, Fujii reported only $18 \%$ compliance of patients. This results in a tremendous amount of leftover medication in homes and communities, available for diversion. ${ }^{54,57,58,59,60}$ This is especially significant in the youth population, who often have unrestricted access to where the medication is stored. ${ }^{27,61,62}$

Various interventions have been recommended to improve disposal. Education has remained a foundation of many studies. ${ }^{58,63,64}$ Hasak and Rose prospectively demonstrated an increase in appropriate disposal from $11 \%$ to $22 \%$ and $5 \%$ to $27 \%$ in surgical patients who received an educational brochure on appropriate disposal. ${ }^{63}$ 
Drug take-back programs, a national prescription drug take-back day, drug kiosks, and mail-in programs have been implemented in some locations often with news releases citing success and tons of medication returned. ${ }^{65,66}$ However, when considering the overall quantity of opioid medication prescribed compared to what is returned, the effectiveness of drug take-back programs is likely small. ${ }^{67,68,69}$

Although there is a lack of knowledge regarding appropriate drug disposal, even with education it remains quite low. Currently, there is little incentive to return unused pills. Other reasons for poor disposal are inherent cost of medication to the consumer, perceived potential for future need and lack of convenient disposal.

\subsection{THE IMPORTANCE OF ANALGESIA IN OUTPATIENT SURGERY}

In outpatient surgery, an adequate analgesia and symptom protocol is extremely relevant as uncontrolled pain, nausea or other symptoms result in longer discharge times and potential hospital stays. ${ }^{71}$ Moderate to severe pain will prolong recovery room stay by $40-80$ minutes. ${ }^{7172}$ Optimal analgesia not only facilitates discharge, but decreases re-admission rates, improves patient satisfaction and facilitates earlier return to function. ${ }^{72}$ Over recent years, outpatient surgery has become increasingly common, and may involve more extensive and painful operations. The role of effective, opioid-sparing, multimodal analgesia thus becomes more critical role in facilitating the discharge and recovery process of these patients.

\subsection{INTERVENTIONS FOR OPIOID REDUCTION AND EFFECTIVE ANALGESIA IN SURGERY}

Evidence-based opioid-reduction strategies after surgery that have been explored include: (1) patient education (2) provider Education (3) opioid-reduced prescriptions (4) non-opioid multimodal analgesia strategies. ${ }^{70}$ 


\subsubsection{PATIENT EDUCATION}

Unfortunately, current analgesic practice is often providing an opioid prescription after surgery with instructions to use as needed for pain. This does not commonly include education about other medical or conservative adjuncts or education about expected levels of discomfort after surgery. This practice results in the following: a) the patient fills the prescription nearly $100 \%$ of the time; b) the patient may take the entire prescription for only mild discomfort; c) the patient may not tolerate or use the opioid, leaving excess within the home $;{ }^{49,50}$ and $\mathrm{d}$ ) the patient is left questioning other analgesic options without instructions on their optimal use, ${ }^{.74}$

In 114 women, Katz demonstrated how pre-operative anxiety was an important variable contributing to the prediction of acute pain after surgery. ${ }^{75}$ Another systematic review evaluating the effectiveness of physician-patient communication and health outcomes, highlighted a positive correlation between effective communication and improved patient health outcomes including analgesia, symptom resolution, function and emotional health. ${ }^{76,77,78}$

Sugai prospectively evaluated a control group in outpatient surgery who received no patient education, to an intervention group that received both oral and written forms of patient education. ${ }^{73}$ This education surrounded patient expectations, natural endorphins, the negative effects of narcotics, and mechanisms of non-opioid analgesics. ${ }^{73}$ Despite $100 \%$ of the control group filling a narcotic prescription vs $10 \%$ of the intervention group, average pain scores were significantly lower in the intervention group. ${ }^{73}$ Perioperative education has been reaffirmed to have a positive correlation with patient satisfaction of analgesia, pain scores, and opioidreduction. ${ }^{76-77,79-81}$ Written instructions have been compared to verbal instructions in patients being discharged from hospital. Studies have revealed that a combination of written and verbal information, particularly on pain relief, are valued by patients. Caregivers also have an integral role in receiving instructions prior to discharge. ${ }^{82}$ In a Cochrane review, parents had a better understanding of the care needed for pediatric patients when given both written and verbal instructions compared to written instructions alone. ${ }^{83}$ 


\subsubsection{PROVIDER EDUCATION}

It is general practice for every patient to receive an opioid prescription after surgery. Surgery is inherently painful and with recent emphasis of pain labeled "the fifth vital sign" and the underestimation of the addiction potential of opioids, excessive opioid prescriptions are often given with the intention of better pain control.

There is now evidence that patients use a fraction of the opioids prescribed and that non-opioid multimodal analgesic medications are often equally effective in controlling post-operative pain. ${ }^{50,51,122}$ This calls for a culture shift, which must start with the surgeons and involve the entire care team.

When Hill discussed findings of the wide variation and excess opioid prescriptions along with actual opioid consumption, opioid prescribing dropped dramatically. ${ }^{84}$ The total number of pills that would have been prescribed decreased by $53 \%{ }^{84}$

Described methods of education include divisional or perioperative rounds, patient quality and safety meetings, emails, educational assist devices (cards) or meeting with each department separately. ${ }^{70}$ Stanek educated providers through educational assist devices (pink cards) which served as a memory prompt and were given to physicians, nurse practitioners and medical trainees. ${ }^{85}$ Howard disseminated practice guidelines to staff though video and oral presentations and reduced opioid prescribing by $38 \% .{ }^{55}$ Hill distributed education through emails, surgery section meetings, resident forums, and grand rounds. ${ }^{84}$

\subsubsection{OPIOID-REDUCED PRESCRIPTIONS}

Prescriptions for opioids may be reduced through the following methods. First, surgeons may be educated on the need for opioid reduction and may use their own judgement to reduce opioid prescriptions. Second, written guidelines on recommended quantity of opioids to prescribe for procedures may be distributed across surgical divisions. ${ }^{84}$ Third, electronic medical records 
(EMRs) may be adjusted to have lower automatic quantities and duration of opioid prescriptions. Finally, rescue-opioid prescriptions may be utilized.

\section{a. Institutional guidelines}

Guidelines created by the Department of Surgery, Michigan Medicine were made available to the different surgical departments based on a previous pre- and post-intervention study comparing opioids prescribed to opioids consumed after surgery. Five months after guideline implementation, the median amount of opioid prescribed was reduced from $250 \mathrm{OME}$ to 75 OME with no change in prescription refills (2.5\%). Median post-operative opioid use had decreased from $30 \mathrm{OME}$ to $20 \mathrm{OME}$. There is always crossover between written guidelines and provider education, however, together they may have a synergistic effect. ${ }^{52,84}$

\section{b. Electronic health systems}

Simply decreasing the default quantity and duration of opioid tablets in an EMR system is another method to decrease opioid prescriptions. ${ }^{85,88,89}$ Luk changed the default opioid prescription from one that automatically offered weight based acetaminophen + codeine or hydrocodone, to an order set with acetaminophen and ibuprofen. ${ }^{90}$ Opioid prescribing decreased from $82 \%$ to $15 \% .^{90}$

\subsubsection{NON-OPIOID MULTIMODAL ANALGESIC STRATEGIES}

It is current general practice to provide most perioperative analgesia through opioid analgesics. While effective analgesic agents, their use must be balanced with well documented side effects including post-operative nausea and vomiting, sedation and falls, ventilatory depression, constipation, ileus, urinary retention and pruritus. ${ }^{91}$

Adequacy of pain control is imperative in determining if a patient can be discharged safely from an ambulatory facility. ${ }^{92}$ It requires an effective perioperative analgesic technique that is intrinsically safe, has minimal side effects and may be easily managed in the home setting. ${ }^{92}$ 
Perioperative multimodal analgesia has been proposed as a safe, inexpensive method to improve pain control and nausea, reduce opioid use, and contribute to a low-risk, opioid-sparing clinical practice. ${ }^{93}$

\subsubsection{PRE-EMPTIVE AND PRE-OPERATIVE MULTIMODAL ANALGESIA}

Recent guidelines in ambulatory surgery have advocated for a multimodal, opioid-sparing analgesic approach in the immediate perioperative period. ${ }^{94-96}$ The concept of pre-emptive analgesia is altering central processing, to decrease the incidence of hyperalgesia after surgery. ${ }^{97}$ The clinical evidence for the timing of administration of pre-emptive medications is debatable as to whether there is more effect if they are given pre- or intra-operatively. ${ }^{98}$

\section{a. Local Anesthetic Nerve Blocks.}

The infiltration of local anesthetic agents, such as lidocaine, in or around the border of a surgical wound have consistently been shown to reduce post-operative pain, and may decrease postoperative opioid use. ${ }^{99,100-102,103}$ When administered during or before surgery, this decreases both analgesic and anesthetic requirements. ${ }^{72}$ For example, an ilioinguinal nerve block, or iliohypogastric nerve block significantly decreased opioid analgesic requirements in patients undergoing inguinal hernia repair by providing 6-8 hours of post-operative analgesia. ${ }^{104}$ There has been recent interest in liposomal bupivacaine, to produce prolonged analgesia with single dose infiltration into the surgical site. In a pooled analysis of 9 studies representing 5 surgical procedures, liposomal bupivacaine was associated with lower pain scores and less consumption

of opioids post-operatively. ${ }^{105,106}$ This medication is not yet licensed in Canada.

\section{b. Regional Anesthetic Nerve Blocks.}

There is an extensive body of literature examining the effectiveness of regional anesthetic techniques to improve analgesia. ${ }^{107}$ These include upper and lower extremity blocks, central neuroaxial, paravertebral blocks and other regional nerve blocks. The majority have evidence for efficacy in post-operative analgesia, however, are also associated with time, cost and 
complications such as hematoma formation. ${ }^{108-112}$ Coupled with the variability due to administrator experience, these factors have led to mixed views on their utility in various outpatient surgery. ${ }^{108-112}$ Certainly, while central neuroaxial (epidural and spinal) blocks provide excellent analgesia, these are not practical techniques in outpatient surgery. ${ }^{72}$

\section{c. NSAIDs}

Pre-emptive use of NSAIDs has been associated with improved analgesia and lower opioid consumption post-operatively. The timing of NSAID administration has been debated and studies have shown effectiveness when given both pre- and intra-operatively. ${ }^{98,113}$

In patients undergoing laparoscopic cholecystectomy, ketorolac has been shown to decrease opioid requirements and improve ventilatory parameters post-operatively. ${ }^{114}$ A meta-analysis of 13 randomized clinical trials evaluated a single peri-operative dose of ketorolac, establishing it as an effective adjunct in multimodal regimens to reduce post-op pain. ${ }^{115}$ It is also therapeutic in reducing post-operative nausea and vomiting. ${ }^{115}$ While many guidelines advocated for preemptive use of NSAIDs, there are challenges to standardizing this practice due to the variability of the anesthetist and suitability of the individual patient.

\section{d. Dexamethasone}

A single perioperative dose of dexamethasone has been shown to be effective in decreasing postoperative pain in a meta-analysis of 45 randomized clinical trials. ${ }^{116,117}$ Opioid consumption has also been noted to decrease with dexamethasone. ${ }^{116,117,118}$ Typical peri-operative doses are between $0.1-0.2 \mathrm{mg} / \mathrm{kg} .{ }^{117}$ Dexamethasone also decreases nausea and vomiting, is an appetite stimulant and may promote a sense of well-being. ${ }^{11-120}$ These positive attributes facilitate the discharge of outpatient procedures. ${ }^{116,117,119,120}$ There is no evidence of delayed wound healing or increased wound infections with a single perioperative dose of dexamethasone. ${ }^{116,117}$ 


\subsubsection{POST-OPERATIVE OPIOID-SPARING MULTIMODAL ANALGESIA}

After outpatient surgery, opioids may be used as rescue therapy, or regularly for a short duration of less than 3 days with immediate tapering and discontinuation. ${ }^{96}$ Rescue therapy is defined as an optional opioid prescription, that may be filled if non-opioid analgesia options do not satisfactorily control the patient's pain. Two recent systematic reviews reaffirmed that opioids consumed in surgery are a fraction of what is prescribed, with patients rarely consuming more than 15 pills. ${ }^{51,50}$ This is likely an over-estimation of the number of opioid pills needed, as many studies did not include instructions for other analgesic options, which may in fact have decreased overall opioid requirements.

\section{a. NSAIDs}

Benefits of NSAIDs include analgesic, anti-inflammatory and antipyretic effects. NSAIDS may be equivalent to opioids in controlling acute post-operative pain. ${ }^{121,122}$ Three clinical trials involving 1202 patients with moderate or severe pain after surgery found that fewer patients needed rescue therapy with ibuprofen alone, than with oxycodone alone. ${ }^{122}$ In another doubleblinded randomized control trial (RCT) in 104 patients, there was equivalent post-operative pain in those given $800 \mathrm{mg}$ of ibuprofen vs $800 \mathrm{mg}$ of acetaminophen with $60 \mathrm{mg}$ of codeine as postoperative prescriptions. ${ }^{121}$ Stessel compared controlled-release oxycodone vs. naproxen in a RCT involving 105 patients and also found no significant difference in patient-reported pain levels. ${ }^{123}$

NSAIDs are safe and effective analgesic agents, especially when used in short duration in an elective non-comorbid population. Caution must be used, however, as they have well documented side effects including gastrointestinal ulceration, renal tubular damage and platelet dysfunction. ${ }^{124}$ There are also concerns of their use in those with severe cardiovascular disease and those patients with high risk of bleeding, although most reviews support that the increase of bleeding is equivocal at best. ${ }^{124,125}$

Meloxicam is one of the only NSAIDs that requires a prescription in Ontario. This is likely due to the risk of cardiovascular thrombotic events, although recent studies suggest it does not 
interfere with platelet aggregation. ${ }^{126}$ It reversibly inhibits cyclooxygenase- 2 which results in decreased formation of prostaglandin precursors and has antipyretic, analgesic, and antiinflammatory properties. ${ }^{127} \mathrm{~A}$ meta-analysis reporting on 12 RCTs suggested that when compared to a non-COX-2 selective NSAIDs, meloxicam resulted in fewer peptic ulcer bleeds, less dyspepsia and less frequent discontinuation related to adverse GI related events. ${ }^{128}$

\section{b. Acetaminophen}

Acetaminophen or paracetamol is a safe, well-tolerated medication that is an effective opioid-

sparing analgesic agent for mild to moderate pain. ${ }^{129-131}$ A maximum daily dose of four grams per day is recommended, however, a special consideration is cirrhotic patients where it should be used in reduced doses (maximum daily dose of two grams). ${ }^{130}$ Acetaminophen is recommended routinely in the post-operative period for both its analgesic and antipyretic properties.

In acute post-operative pain, a Cochrane review of 51 studies with 5,762 patients found that a single dose of acetaminophen reduced patient-reported pain and was associated with few adverse events. ${ }^{130}$ Current evidence suggests that acetaminophen in combination with an NSAID work synergistically to offer superior analgesia than with either drug alone. ${ }^{129,132}$

\section{c. NMDA antagonists, Alpha-2 agonists, Gabapentinoids, Selective Serotonin Reuptake Inhibitors and Tricyclic Antidepressants}

Ketamine, amantadine and dextromethorphan are NMDA receptor antagonists that have been used in the setting of palliative care, chronic and neuropathic pain, and may be an adjunct in the treatment of post-surgical pain. ${ }^{72,133}$ There is mixed evidence regarding their benefit as ketamine had previously fallen out of favour due to its side effect profile. ${ }^{72}$ A recent review, however, of 5 meta-analyses and 39 clinical trials founds that ketamine does reduce opioid consumption although its utility in lowering pain scores is less clear. ${ }^{134-136}$ Its use may be more amenable for patients with a history of chronic opioid use, in which ketamine or amantadine are an adjunct for decreasing opioid consumption and may contribute to post-operative analgesia. ${ }^{135}$ Due to its intravenous form and often being given as a slow infusion post-operatively, ketamine has more 
of a role in major surgical procedures than outpatient procedures. ${ }^{72,134}$ While ketamine is generally considered a safe drug, there are concerns for possible neurotoxicity and effects on memory. ${ }^{136}$ The use of smaller doses has resulted in less frequent adverse events and greater physician acceptance. ${ }^{72}$

Dexmedetomidine, clonidine and tizanidine are alpha-2 agonists which possess anxiolytic and sedative properties due to their central antinociceptive activity. ${ }^{72,137}$ A Cochrane Review in 2016 examining dexmedetomidine in 422 patients from 7 RCTs after abdominal surgery showed no reduction in pain scores, but decreased breakthrough opioid consumption in the first 24 hours. ${ }^{133,137}$ In a meta-analysis of clonidine in 2012 of 19 RCTs, there was an opioid-sparing effect within 24 hours, although less pronounced than dexmedetomidine. ${ }^{133,138}$ Since this time, other studies have reproduced similar results. ${ }^{139}$ Tizanidine, differs in its shorter duration of action, with less systemic effects and may result in less post-operative pain after surgery, although there is much less published data. ${ }^{140}$

Methocarbamol, cyclobenzaprine, clonidine and tizanidine all have muscle relaxing properties. ${ }^{133}$ This muscle-relaxing effect may be responsible for some opioid reduction. More evidence is needed, particularly with methocarbamol and cyclobenzaprine as to the role of muscle relaxants as analgesic adjuncts, before they can be routinely recommended post-operatively. ${ }^{133,138,140}$

Gabapentinoids (gabapentin and pregabalin), were initially developed as anticonvulsant medications. ${ }^{133}$ Now used more commonly for chronic neuropathic pain, their uses have been expanded to post-surgical pain, suggesting effects of opioid-reduction and the prevention of chronic pain syndromes. ${ }^{72,133,141,142}$ Most meta-analyses concur their use results in a decrease in opioid consumption, although there are various reports on their actual reduction of post-operative pain scores. ${ }^{143,144}$ Higher-quality studies using better grading of articles included and metaregression suggest the extent of opioid-reduction is likely overestimated. ${ }^{145,146}$ In his metaanalysis with trial sequential analysis, Doleman reported that in 13 trials (with low risk of bias) of 132 trials comparing gabapentin vs. placebo there was a reduction of 24 hour opioid consumption equivalent to $3.1 \mathrm{mg}$ of morphine. ${ }^{146}$ Given the predefined minimal clinically significant effect of morphine is $5 \mathrm{mg}$ in 24 hours, their conclusion was there is an absence of 
any clinically relevant beneficial effect of gabapentin and harm may be pending. ${ }^{146}$ The dosing of pregabalin is more predictable and the majority of meta-analyses also conclude a decrease in opioid consumption. ${ }^{147-149}$ In 2015 a meta-analyses by Eipe included 43 studies using pregabalin for acute pain which demonstrated a $16 \%$ reduction in analgesic consumption along with a questionable small reduction in pain scores. ${ }^{147}$ Sedation, dizziness and blurred vision were relatively common. ${ }^{147}$ In a meta-analysis involving 55 studies in 2015 by Mishriky, pregabalin also reduced opioid consumption at 24 hours in addition to pain scores, however, side effects were common. Data was insufficient to reach any conclusions regarding persistent pain. ${ }^{148}$ Firm evidence for the routine use of routine gabapentin and pregabalin is still lacking, especially considering the current minimal benefit in pain control and the well-documented side effects. ${ }^{146}$

Commonly used in depression and anxiety there has been recent interest in serotonin norepinephrine reuptake inhibitors (eg. Duloxetine) as analgesic adjuncts. ${ }^{107}$ Although limited evidence suggests there may be an opioid sparing effect, it does not consistently reduce postoperative pain scores when compared to placebo. ${ }^{107}$ The opioid-sparing effects reported are modest, and more evidence is needed to determine the role of SNRI's in the treatment of acute postoperative pain. ${ }^{150-152}$

Tricyclic antidepressants (amitriptyline and desipramine) are commonly used to treat chronic pain conditions, and their role has been questioned in the management of acute post-operative pain. 153 This evidence is sparse with Kerrick and Vahedi reporting amitriptyline as an adjunct to opioids was no more effective than placebo in controlling pain. ${ }^{153-158}$ Considering other evidence remains ambiguous with minimal if any effect, while TCAs have an integral role in the management of chronic pain conditions, their routine use in acute post-operative pain is currently not recommended.

\subsubsection{NON-PHARMACOLOGIC TECHNIQUES}

Other nonpharmacologic techniques discussed in the literature are transcutaneous electrical nerve stimulation, acupuncture, cryoanalgesia, ultrasound, laser stimulation and hypnotherapy. Well- 
designed clinical trials are needed prior to the recommendation of these strategies to establish the benefits on post-operative pain. ${ }^{72}$

Cryoanalgesia or ice therapy has been advocated for soft tissue injuries since the time of Hippocrates. An RCT by Koc analyzed ice therapy at 2, 6, and 24 hrs after the operation. There were significant differences in the visual analogue pain scale ratings between the groups and they concluded it was a safe, effective technique for providing analgesia following inguinal hernia repair. ${ }^{159}$ Another RCT published in the Journal of the American College of Surgeons assessed ice therapy in patients who had midline laparotomies and concluded that both opioid use and pain were lower in the cryotherapy group. ${ }^{160}$ There is some concern that ice therapy may cause skin flap necrosis, however these are infrequent case reports. While there is minimal evidence for ice therapy in breast surgery, it is reasonable to extrapolate data from other surgical procedures and hypothesize it may be effective in reducing both pain and opioid use.

\subsubsection{CHRONIC POST-SURGICAL PAIN}

As one predictor of chronic pain syndromes after surgery is acute pain, effective analgesia in the post-operative period is consequential. Other predictors include age, sex, genetics and preoperative pain. ${ }^{156}$ Biologic, environmental mechanisms and psychosocial mechanisms all play a role in the development of chronic pain. ${ }^{157}$ Current peri-operative pharmacologic interventions which may have evidence for suppressing and preventing sensitization and development of chronic pain include NSAIDs, ketamine, local anesthetics and the use of multimodal analgesia. ${ }^{157,158}$ The use of gabapentin, pregabalin, TCAs and SNRIs to prevent chronic pain syndromes after surgery is sparse and weak, and needs to be further evaluated. ${ }^{157,158}$

\section{6 "BUNDLES" IN SURGERY}

The concept of "bundles" has been introduced in surgery through combining multiple evidencebased strategies to obtain a greater overall and often synergistic effect. Enhanced recovery after surgery (ERAS) is an example and centers on a multidisciplinary team approach in developing 
evidence-based, patient centered pathways to optimize the patients' function and recovery. ${ }^{161}$ This is often an integrated continuum, from the pre-hospital stay until discharge and has revolutionized the recovery process after surgery by reducing post-operative care time by more than $30 \%$ and reducing complications by up to $50 \% .{ }^{162}$ These "bundles" have been focused primarily on inpatient procedures, however, may also be effective in the outpatient setting. ${ }^{162-165}$ Minimizing opioids is one of the many multi-pronged strategies of ERAS protocol, but there is a lack of current guidelines and recommendations as to the quantity and duration of post-operative opioids to prescribe, as well as the integration of multimodal analgesia in the outpatient setting.

\subsection{OBJECTIVES AND PURPOSE OF THE THESIS}

\subsubsection{DEFINING THE PROBLEM IN BREAST AND ANORECTAL SURGERY}

The scope of the opioid crisis in North America has been outlined above. The evidence of the potential roles of the surgeon in the opioid prescription problem has also been explored.

There is a lack of current guidelines and recommendations as to the quantity and duration of post-operative opioids to prescribe in outpatient anorectal and breast surgery. These procedures were chosen as they are almost uniformly performed as outpatient procedures. This surgical cohort represented a population suitable to expand recently studied standardized opioid-reduction protocols which were successful in other outpatient procedures at LHSC(Appendix 5.5) ${ }^{57}$ In addition, while opioid-reducing interventions have been studied there are few examining the feasibility of combining these interventions together in a bundle in these patients. Furthermore, the majority of literature on opioid reduction often includes prescription refills as an indirect marker for adequate control of pain rather than patient-reported pain scores.

Considering there are over 20,000 outpatient breast surgeries and a significant number of anorectal surgeries in the province of Ontario per year, the STOP Narcotics initiative could potentially decrease opioids and risk of opioid misuse after surgery impacting the opioid crisis in a truly meaningful way. 
The Division of General Surgery established Opioid Prescribing for Acute Pain as a priority area for improvement by the Division of General Surgery, Schulich School of Medicine and Dentistry at Western University. This was based on the 2017 Health Canada - Joint Statement of Action to Address the Opioid Crisis and published evidence on the wide variation and excess prescription of opioids in general surgery ${ }^{23}$

\subsubsection{ORTHOPEDIC EDUCATIONAL RANDOMIZED CONTROLLED TRIAL AT LONDON HEALTH SCIENCES CENTRE}

An RCT by Singh from the Division of Orthopedic Surgery at Western University provided the motivation and foundation for subsequent work on opioid reduction completed in opioidreduction by the Division of General Surgery. ${ }^{166}$ In 2016 this orthopedic RCT evaluated the role of a post-operative pain guideline pamphlet on patient's pain satisfaction scores. While there was no significant difference noted in satisfaction, only 7/78 (9\%) used the entire opioid prescription and 5/78 (6\%) required a prescription refill. Despite 41 patients receiving specific instructions regarding disposal, only 5 patients returned their surplus medication to pharmacy. ${ }^{166}$

\subsubsection{DEVELOPMENT OF THE STOP NARCOTICS INITIATIVE}

The STandardization of Outpatient Procedure Narcotics (STOP Narcotics) initiative was developed in response to defining the problem of opioids in surgery and the role of the surgeon in the opioid crisis. This was a division-wide, multidisciplinary health systems plan that focused on adequately controlling patients' pain through a multipronged approach.

The STOP Narcotics initiative involved the implementation of a 4-pronged strategy: patient and care-giver education through counseling and written materials; health care provider education (surgeons, anaesthetists, residents, and nurses); intra-operative multi-modal analgesia; and postoperative multi-modal opioid reduction strategies for pain control. The protocol was designed with input from relevant stakeholders (surgeons, anesthetists, nurses, and patients) and was instituted across the Division of General Surgery at Western University. 


\subsubsection{HERNIA REPAIR AND LAPAROSCOPIC CHOLECYSTECTOMY}

As part of a prior project, the STOP Narcotics initiative was implemented in a pre- and postintervention study including elective, outpatient laparoscopic cholecystectomy and open hernia (inguinal, ventral) patients. A control cohort of 224 patients was compared to a post-intervention group of 192 patients. Average post-operative pain scores in the pre- vs post-intervention group was equivalent. The multipronged intervention was associated with a 50\% reduction in opioid prescribing with only $45 \%$ of patients filling their prescription (Table 1.2).

Table 1.2 Outcomes of outpatient hernia and laparoscopic cholecystectomy

\begin{tabular}{|cc|}
\hline \multicolumn{2}{|c|}{ Ventral/Umbilical/Inguinal Hernias and Laparoscopic Cholecystectomies } \\
\hline Average pain in first 7 post-op days, mean (SD) & (Equivalent reported pain NRS [1-10]) \\
\hline OME, median & (50\% reduction in opioid prescribing) \\
Narcotic prescription filled, $\mathrm{n}(\%)$ & (Only $45 \%$ filled their prescription) \\
\hline
\end{tabular}

\subsubsection{RETROSPECTIVE REVIEW OF PRESCRIBING PRACTICES}

The work of this thesis on breast and anorectal outpatient surgery patients was built upon this foundation of the hernia and laparoscopic cholecystectomy study. To further define the problem in breast and anorectal surgeries, we collected data retrospectively over a 3-month time period at London Health Sciences Centre (LHSC) and St. Joseph's Health Care (SJHC) examining prescription practices (Figure 1.3). Moderate quantities of opioid medications were being prescribed after these operations.

Figure 1.3 


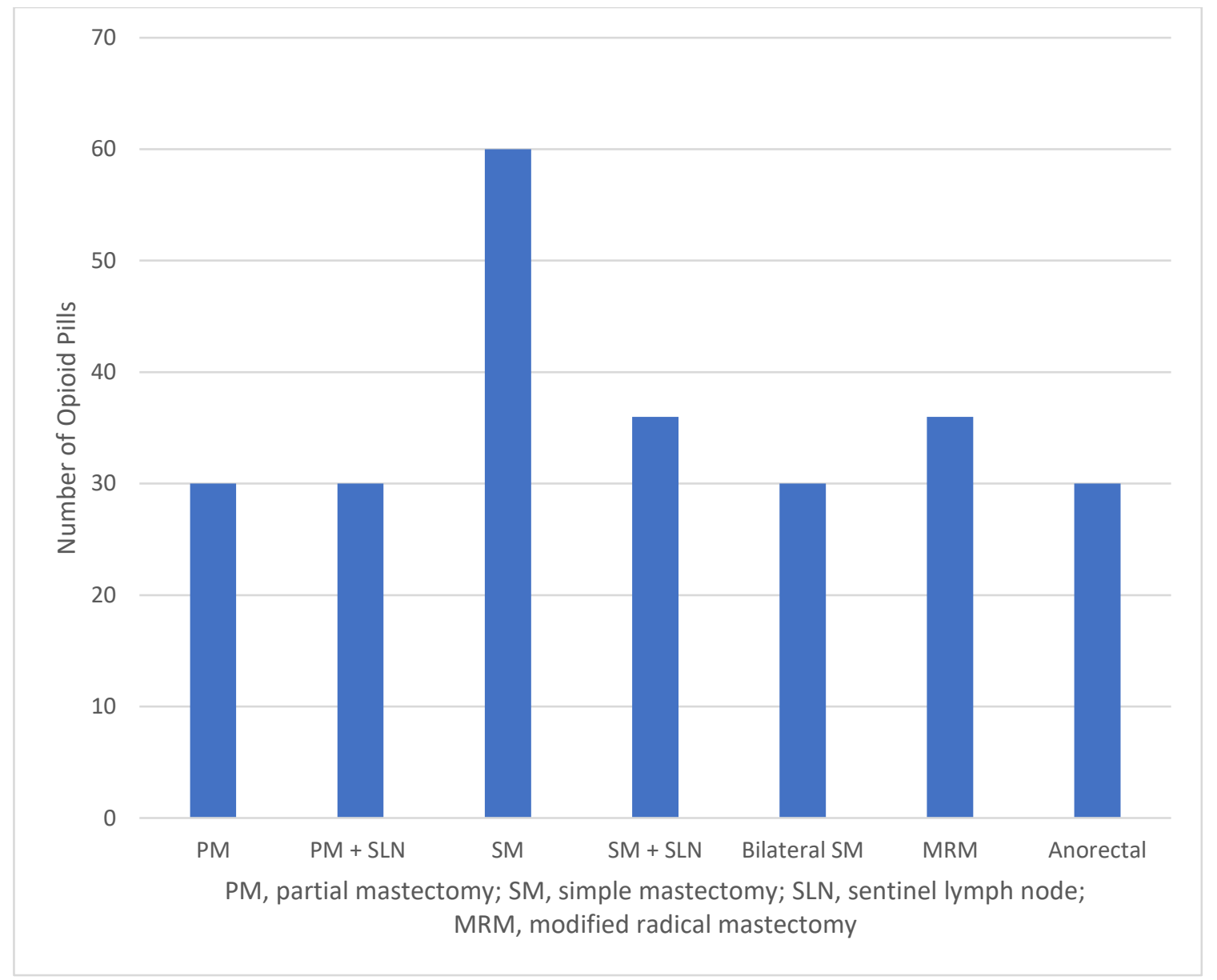

Figure 1.3 Retrospective data of median opioid pills prescribed in 145 outpatient breast and 56 outpatient anorectal surgeries over 3 months at LHSC and SJHC.

This data was compared to Hill who quantified the average number of opioid pills prescribed after partial mastectomy (PM) as 20, with only $15 \%$ (3 pills) being consumed. As we were prescribing a median of 30 pills for PM, this confirmed overprescribing in breast surgery as this was roughly 10 times the amount being consumed, leaving a great amount of excess medication. ${ }^{49}$ These excess and variable prescriptions were paralleled in the other breast surgical and anorectal procedures at our institution.

In further examining this retrospective data, the type of opioid medication prescribed varied significantly in both breast surgery and anorectal surgery, with tramacet being the most commonly prescribed opioid in both populations. 
Figure 1.4

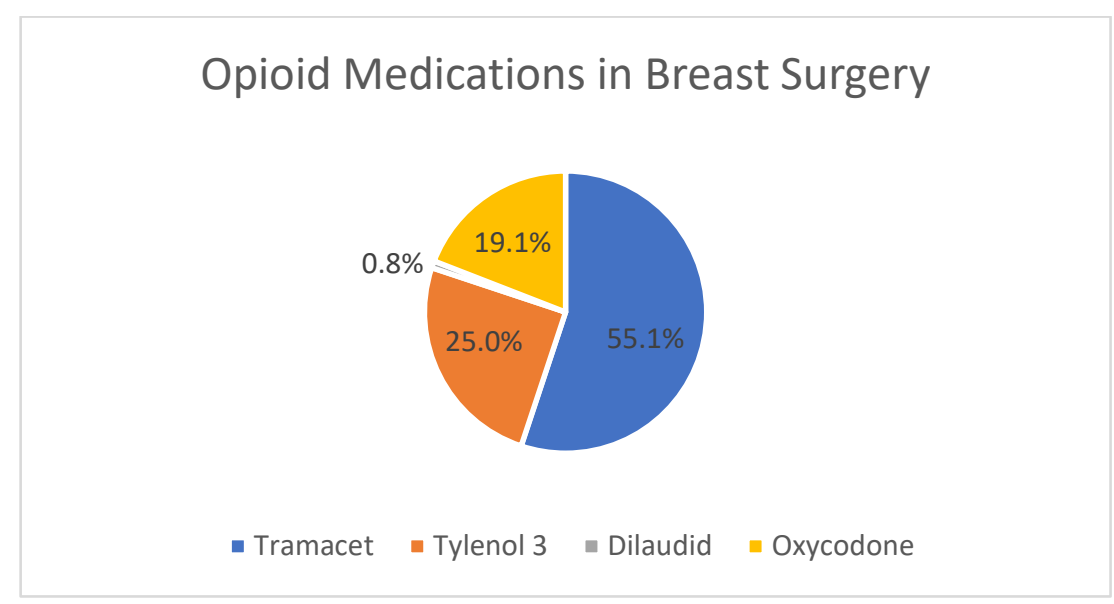

Figure 1.4 Opioid prescriptions by type in 145 breast surgery patients

Figure 1.5

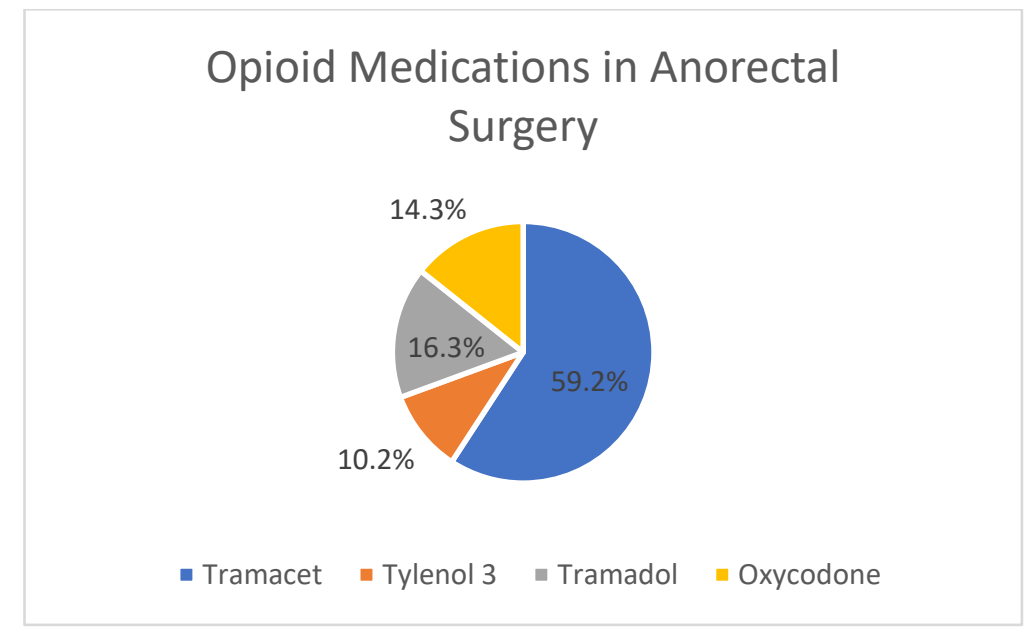

Figure 1.5 Opioid prescriptions by type in 56 anorectal surgery patients

The methodology of the STOP Narcotics initiative in breast and anorectal procedure is similar to the previously published work in the Journal of the American College of Surgeons on laparoscopic cholecystectomy and hernia patients (Appendix 5.5). ${ }^{57}$ The patient care bundles 
presented in this thesis are tailored to the breast and anorectal surgery population and their unique pain considerations. Therefore, expanding the work from the original STOP Narcotics initiative to investigate patients undergoing breast and anorectal procedures was the objective of this current thesis.

This study was designed as a non-inferiority study for the primary outcome of pain control as we postulated surgeons would only change their practice if we demonstrated that there was "not unacceptably worse" pain control than the standard of care. A pre- and post- study is also a practical quality improvement design that is appropriate to use when the post-intervention group is not expected to change in terms of population, outcomes, or practices. As our inclusion

criteria, surgical practices, and outcomes (e.g. patient's rating of pain) were not expected to change in the post-intervention group, we proposed this design may result in less overall variability. Practically, a pre- and post- design also leads to a better compliance rate leading to more consistent interventions and practice. Considering there was a strong feeling in the department of general surgery that practice change in opioid prescribing needed to occur, this design allowed us to smoothly transition the intervention to routine practice after study completion.

\subsection{PURPOSE STATEMENT AND HYPOTHESES}

The purpose of the study was to modify the standardized multi-pronged, opioid-sparing, divisional intervention (STOP Narcotics) to adequately control post-operative pain, while reducing excessive post-operative opioid prescriptions in outpatient breast and anorectal surgical procedures.

We hypothesized that with the introduction of the STOP Narcotics protocol, non-inferior pain control may be obtained while reducing narcotic prescriptions amongst patients undergoing these selected common outpatient general surgical procedures.

\subsection{REFERENCES}

1. Inturrisi CE. Clinical Pharmacology of Opioids for Pain. Clin J Pain. 2002;18(4):S3-S13. 
https://insights.ovid.com/clinical-pain/cjpn/2002/07/001/clinical-pharmacology-opioidspain/2/00002508. Accessed December 30, 2018.

2. Benyamin R, Trescot AM, Datta S, et al. Opioid complications and side effects. Pain Physician. 2008;11(2 Suppl):S105-20. http://www.ncbi.nlm.nih.gov/pubmed/18443635. Accessed December 30, 2018.

3. Svendsen K, Borchgrevink P, Fredheim O, et al. Choosing the unit of measurement counts: The use of oral morphine equivalents in studies of opioid consumption is a useful addition to defined daily doses. Palliat Med. 2011;25(7):725-732. doi:10.1177/0269216311398300

4. Pathan H, Williams J. Basic opioid pharmacology: an update. Br J pain. 2012;6(1):11-16. doi:10.1177/2049463712438493

5. Diagnostic and Statistical Manual of Mental Disorders. Fifth Edit. Arlington, VA: American Psychiatric Association; 2013

6. Kosten TR, George TP. The neurobiology of opioid dependence: implications for treatment. Sci Pract Perspect. 2002;1(1):13-20. http://www.ncbi.nlm.nih.gov/pubmed/18567959. Accessed December 30, 2018.

7. Lyapustina T, Alexander C. The prescription opioid addiction and abuse epidemic: how it happened and what we can do about it. Pharm J. 2015. doi:10.1211/PJ.2015.20068579

8. United Nations on Drugs and Crime: World Drug Report 2018. https://www.unodc.org/wdr2018/. Accessed December 30, 2018.

9. World Health Organization: Information sheet on opioid overdose. https://www.who.int/substance_abuse/information-sheet/en/. Accessed December 30, 2018.

10. Board INC. Narcotics Drugs: Estimated World Requirements for 2013 - Statistics for 2011. New York; 2011.

11. Centers for Disease Control and Prevention. Opioid Overdose: Understanding the Epidemic. https://www.cdc.gov/drugoverdose/epidemic/index.html. Published 2018. Accessed December 30, 2018.

12. Fischer B, Argento E. Prescription opioid related misuse, harms, diversion and interventions in Canada: a review. Pain Physician. 2012;15(3 Suppl):ES191-203. http://www.ncbi.nlm.nih.gov/pubmed/22786457. Accessed December 31, 2018.

13. Compton WM, Volkow ND. Major increases in opioid analgesic abuse in the United States: Concerns and strategies. Drug Alcohol Depend. 2006;81(2):103-107. doi:10.1016/J.DRUGALCDEP.2005.05.009 
14. Canadian Centre on Substance Use. Prescription Opioids (Canadian Drug Summary).; 2017. www.ccsa.ca•www.ccdus.ca. Accessed December 30, 2018.

15. Seth P, Scholl L, Rudd RA, Bacon S. Overdose Deaths Involving Opioids, Cocaine, and Psychostimulants — United States, 2015-2016. MMWR Morb Mortal Wkly Rep. 2018;67(12):349-358. doi:10.15585/mmwr.mm6712a1

16. National Center for Health Statistics. Wide-Ranging Online Data for Epidemiologic Research (WONDER). Atlanta, GA; 2017. http://wonder.cdc.gov.

17. Belzak L, Halverson J. Evidence synthesis - The opioid crisis in Canada: a national perspective. Heal Promot Chronic Dis Prev Canada. 2018;38(6):224-233. doi:10.24095/hpcdp.38.6.02

18. Public Health Ontario. Opioid-related morbidity and mortality in Ontario. https://www.publichealthontario.ca/en/dataandanalytics/pages/opioid.aspx. Published 2018. Accessed December 31, 2018.

19. News Global. 341 traffic fatalities on OPP patrolled roads in 2017 in Ontario. https://globalnews.ca/news/3941702/ontario-traffic-fatalities-2017-opp/.

20. Health Quality Ontario. 9 Million Prescriptions: What We Know about the Growing Use of Prescription Opioids in Ontario.; 2017.

21. Young L. The Impact of Prescription and Non-Prescription Drug Use in MiddlesexLondon. 2014. https://www.healthunit.com/uploads/2014-05-15-report-032-14-appendixa.pdf. Accessed December 10, 2017.

22. MAXWELL JC. The prescription drug epidemic in the United States: A perfect storm. Drug Alcohol Rev. 2011;30(3):264-270. doi:10.1111/j.1465-3362.2011.00291.x

23. Van Zee A. The promotion and marketing of oxycontin: commercial triumph, public health tragedy. Am J Public Health. 2009;99(2):221-227. doi:10.2105/AJPH.2007.131714

24. Sherry L. Murphy, B.S., Jiaquan Xu, M.D., Kenneth D. Kochanek, M.A., Sally C. Curtin, M.A. A, Elizabeth Arias PD. National Vital Statistics Reports. https://www.cdc.gov/nchs/data/nvsr/nvsr66/nvsr66_06.pdf.

25. Canadian Institute for Health Information. Amount of opioids prescribed dropping in Canada; prescriptions on the rise. https://www.cihi.ca/en/amount-of-opioids-prescribeddropping-in-canada-prescriptions-on-the-rise. Published 2018. Accessed December 31, 2018.

26. Dhalla IA, Mamdani MM, Sivilotti MLA, Kopp A, Qureshi O, Juurlink DN. Prescribing of opioid analgesics and related mortality before and after the introduction of long-acting 
oxycodone. CMAJ. 2009;181(12):891-896. doi:10.1503/cmaj.090784

27. Substance Abuse and Mental Health Services Administration. National Survey on Drug Use and Health.; 2018. https://www.samhsa.gov/data/data-we-collect/nsduh-nationalsurvey-drug-use-and-health. Accessed December 31, 2018.

28. Jones CM, Paulozzi LJ, Mack KA. Sources of Prescription Opioid Pain Relievers by Frequency of Past-Year Nonmedical Use. JAMA Intern Med. 2014;174(5):802. doi:10.1001/jamainternmed.2013.12809

29. Bartels K, Binswanger IA, Hopfer CJ. Sources of Prescription Opioids for Nonmedical Use. J Addict Med. 2016;10(2):134. doi:10.1097/ADM.0000000000000192

30. Jordan AE, Blackburn NA, Des Jarlais DC, Hagan H. Past-year prevalence of prescription opioid misuse among those 11 to 30 years of age in the United States: A systematic review and meta-analysis. J Subst Abuse Treat. 2017;77:31-37. doi:10.1016/j.jsat.2017.03.007

31. Compton WM, Jones CM, Baldwin GT. Relationship between Nonmedical PrescriptionOpioid Use and Heroin Use. Longo DL, ed. N Engl J Med. 2016;374(2):154-163. doi:10.1056/NEJMra1508490

32. Callinan CE, Neuman MD, Lacy KE, Gabison C, Ashburn MA. The Initiation of Chronic Opioids: A Survey of Chronic Pain Patients. J Pain. 2017;18(4):360-365. doi:10.1016/j.jpain.2016.11.001

33. Deyo RA, Hallvik SE, Hildebran C, et al. Association Between Initial Opioid Prescribing Patterns and Subsequent Long-Term Use Among Opioid-Naïve Patients: A Statewide Retrospective Cohort Study. J Gen Intern Med. 2017;32(1):21-27. doi:10.1007/s11606016-3810-3

34. Brummett CM, Waljee JF, Goesling J, et al. New Persistent Opioid Use After Minor and Major Surgical Procedures in US Adults. JAMA Surg. 2017;152(6):e170504. doi:10.1001/jamasurg.2017.0504

35. Alam A, Gomes T, Zheng H, Mamdani MM, Juurlink DN, Bell CM. Long-term Analgesic Use After Low-Risk Surgery. Arch Intern Med. 2012;172(5):425. doi:10.1001/archinternmed.2011.1827

36. Clarke H, Soneji N, Ko DT, Yun L, Wijeysundera DN. Rates and risk factors for prolonged opioid use after major surgery: population based cohort study. BMJ. 2014;348:g1251. doi:10.1136/bmj.g1251

37. Sun EC, Darnall BD, Baker LC, Mackey S. Incidence of and Risk Factors for Chronic Opioid Use Among Opioid-Naive Patients in the Postoperative Period. JAMA Intern Med. 2016;176(9):1286. doi:10.1001/jamainternmed.2016.3298

38. Marcusa DP, Mann RA, Cron DC, et al. Prescription Opioid Use among Opioid-Naive 
Women Undergoing Immediate Breast Reconstruction. Plast Reconstr Surg. 2017;140(6):1081-1090. doi:10.1097/PRS.0000000000003832

39. Lee JS-J, Hu HM, Edelman AL, et al. New Persistent Opioid Use Among Patients With Cancer After Curative-Intent Surgery. J Clin Oncol. 2017;35(36):4042-4049. doi:10.1200/JCO.2017.74.1363

40. Noble M, Tregear SJ, Treadwell JR, Schoelles K. Long-Term Opioid Therapy for Chronic Noncancer Pain: A Systematic Review and Meta-Analysis of Efficacy and Safety. J Pain Symptom Manage. 2008;35(2):214-228. doi:10.1016/j.jpainsymman.2007.03.015

41. National Quality Forum. Surgery 2015-2017 Final Report. https://www.qualityforum.org/Publications/2017/04/Surgery_20152017_Final_Report.aspx. Published 2017. Accessed January 1, 2019.

42. Hall MJ, Schwartzman A, Zhang J, Liu X. Ambulatory Surgery Data From Hospitals and Ambulatory Surgery Centers: United States, 2010. Natl Health Stat Report. 2017;(102):115. http://www.ncbi.nlm.nih.gov/pubmed/28256998. Accessed January 1, 2019.

43. Martin BC, Fan M-Y, Edlund MJ, DeVries A, Braden JB, Sullivan MD. Long-Term Chronic Opioid Therapy Discontinuation Rates from the TROUP Study. J Gen Intern Med. 2011;26(12):1450-1457. doi:10.1007/s11606-011-1771-0

44. Goesling J, Moser SE, Zaidi B, et al. Trends and predictors of opioid use after total knee and total hip arthroplasty. Pain. 2016;157(6):1259-1265.

doi:10.1097/j.pain.0000000000000516

45. Brummett CM, Janda AM, Schueller CM, et al. Survey Criteria for Fibromyalgia Independently Predict Increased Postoperative Opioid Consumption after Lowerextremity Joint Arthroplasty. Anesthesiology. 2013;119(6):1434-1443.

doi:10.1097/ALN.0b013e3182a8eb1f

46. Boscarino JA, Rukstalis M, Hoffman SN, et al. Risk factors for drug dependence among out-patients on opioid therapy in a large US health-care system. Addiction. 2010;105(10):1776-1782. doi:10.1111/j.1360-0443.2010.03052.x

47. Canadian Institute for Health Information. Hospital Trends in Canada-Results of a Project to Create a Historical Series of Statistical and Financial Data for Canadian Hospitals Over Twenty-Seven Years.; 2005.

https://secure.cihi.ca/free_products/Hospital_Trends_in_Canada_e.pdf. Accessed January $1,2019$.

48. Practice Guidelines for Acute Pain Management in the Perioperative Setting. Anesthesiology. 2012;116(2):248-273. doi:10.1097/ALN.0b013e31823c1030

49. Hill M V., McMahon ML, Stucke RS, Barth RJ. Wide Variation and Excessive Dosage of 
Opioid Prescriptions for Common General Surgical Procedures. Ann Surg. 2017;265(4):709-714. doi:10.1097/SLA.0000000000001993

50. Bicket MC, Long JJ, Pronovost PJ, Alexander GC, Wu CL. Prescription Opioid Analgesics Commonly Unused After Surgery: A Systematic Review. JAMA Surg. 2017;152(11):1066. doi:10.1001/jamasurg.2017.0831

51. Feinberg AE, Chesney TR, Srikandarajah S, Acuna SA, McLeod RS, Best Practice in Surgery Group. Opioid Use After Discharge in Postoperative Patients. Ann Surg. 2018;267(6):1056-1062. doi:10.1097/SLA.0000000000002591

52. Hill M V., Stucke RS, Billmeier SE, Kelly JL, Barth RJ. Guideline for Discharge Opioid Prescriptions after Inpatient General Surgical Procedures. J Am Coll Surg. 2018;226(6):996-1003. doi:10.1016/j.jamcollsurg.2017.10.012

53. Tan WH, Yu J, Feaman S, et al. Opioid Medication Use in the Surgical Patient: An Assessment of Prescribing Patterns and Use. J Am Coll Surg. 2018;227(2):203-211. doi:10.1016/j.jamcollsurg.2018.04.032

54. Fujii MH, Hodges AC, Russell RL, et al. Post-Discharge Opioid Prescribing and Use after Common Surgical Procedure. J Am Coll Surg. 2018;226(6):1004-1012. doi:10.1016/j.jamcollsurg.2018.01.058

55. Howard R, Waljee J, Brummett C, Englesbe M, Lee J. Reduction in Opioid Prescribing Through Evidence-Based Prescribing Guidelines. JAMA Surg. 2018;153(3):285. doi:10.1001/jamasurg.2017.4436

56. Center for Drug Evaluation and Research; U.S. Food and Drug Administration. Safe Disposal of Medicines - Disposal of Unused Medicines: What You Should Know. U.S. Food \& Drug Administration https://www.fda.gov/drugs/resourcesforyou/consumers/buyingusingmedicinesafely/ensuri ngsafeuseofmedicine/safedisposalofmedicines/ucm186187.htm\#1. Accessed January 4, 2019.

57. Hartford LB, Van Koughnett JAM, Murphy PB, et al. Standardization of Outpatient Procedure (STOP) Narcotics: A Prospective Non-Inferiority Study to Reduce Opioid Use in Outpatient General Surgical Procedures. J Am Coll Surg. 2019;228(1):81-88.e1. doi:10.1016/j.jamcollsurg.2018.09.008

58. Hasak JM, Roth Bettlach CL, Santosa KB, Larson EL, Stroud J, Mackinnon SE. Empowering Post-Surgical Patients to Improve Opioid Disposal: A Before and After Quality Improvement Study. J Am Coll Surg. 2018;226(3):235-240.e3. doi:10.1016/j.jamcollsurg.2017.11.023

59. Bates C, Laciak R, Southwick A, Bishoff J. Overprescription of Postoperative Narcotics: A Look at Postoperative Pain Medication Delivery, Consumption and Disposal in 
Urological Practice. J Urol. 2011;185(2):551-555. doi:10.1016/j.juro.2010.09.088

60. Bartels K, Mayes LM, Dingmann C, Bullard KJ, Hopfer CJ, Binswanger IA. Opioid Use and Storage Patterns by Patients after Hospital Discharge following Surgery. Costigan M, ed. PLoS One. 2016;11(1):e0147972. doi:10.1371/journal.pone.0147972

61. Voepel-Lewis T, Wagner D, Tait AR. Leftover Prescription Opioids After Minor Procedures. JAMA Pediatr. 2015;169(5):497. doi:10.1001/jamapediatrics.2014.3583

62. Lankenau SE, Teti M, Silva K, Bloom JJ, Harocopos A, Treese M. Initiation into prescription opioid misuse amongst young injection drug users. Int J Drug Policy. 2012;23(1):37-44. doi:10.1016/J.DRUGPO.2011.05.014

63. Rose P, Sakai J, Argue R, Froehlich K, Tang R. Opioid information pamphlet increases postoperative opioid disposal rates: a before versus after quality improvement study. Can J Anesth Can d'anesthésie. 2016;63(1):31-37. doi:10.1007/s12630-015-0502-0

64. de la Cruz M, Reddy A, Balankari V, et al. The Impact of an Educational Program on Patient Practices for Safe Use, Storage, and Disposal of Opioids at a Comprehensive Cancer Center. Oncologist. 2017;22(1):115-121. doi:10.1634/theoncologist.2016-0266

65. DEA'S Prescription Drug Take-Back Effort-- A Big Success. https://www.dea.gov/pressreleases/2015/10/01/deas-prescription-drug-take-back-effort-big-success-0. Published 2015. Accessed January 4, 2019.

66. Walgreens Leads Fight Against Prescription Drug Abuse with New Programs to Help Curb Misuse of Medications and the Rise in Overdose Deaths | Walgreens Newsroom. https://news.walgreens.com/press-releases/general-news/walgreens-leads-fight-againstprescription-drug-abuse-with-new-programs-to-help-curb-misuse-of-medications-and-therise-in-overdose-deaths.htm. Published 2016. Accessed January 4, 2019.

67. Herring ME, Shah SK, Shah SK, Gupta AK. Current regulations and modest proposals regarding disposal of unused opioids and other controlled substances. J Am Osteopath Assoc. 2008;108(7):338-343. http://www.ncbi.nlm.nih.gov/pubmed/18648027. Accessed January 4, 2019.

68. Egan KL, Gregory E, Sparks M, Wolfson M. From dispensed to disposed: evaluating the effectiveness of disposal programs through a comparison with prescription drug monitoring program data. Am J Drug Alcohol Abuse. 2017;43(1):69-77. doi:10.1080/00952990.2016.1240801

69. Haffajee RL, Jena AB, Weiner SG. Mandatory use of prescription drug monitoring programs. JAMA. 2015;313(9):891-892. doi:10.1001/jama.2014.18514

70. Wetzel M, Hockenberry J, Raval M V. Interventions for Postsurgical Opioid Prescribing. JAMA Surg. 2018;153(10):948. doi:10.1001/jamasurg.2018.2730 
71. Pavlin DJ, Chen C, Penaloza DA, Polissar NL, Buckley FP. Pain as a factor complicating recovery and discharge after ambulatory surgery. Anesth Analg. 2002;95(3):627-34, table of contents. http://www.ncbi.nlm.nih.gov/pubmed/12198050. Accessed January 11, 2019.

72. White PF. The Changing Role of Non-Opioid Analgesic Techniques in the Management of Postoperative Pain. Anesth Analg. 2005;101(Supplement):S5-S22. doi:10.1213/01.ANE.0000177099.28914.A7

73. Sugai DY, Deptula PL, Parsa AA, Don Parsa F. The importance of communication in the management of postoperative pain. Hawaii J Med Public Health. 2013;72(6):180-184. http://www.ncbi.nlm.nih.gov/pubmed/23795326. Accessed January 5, 2019.

74. Owen H, McMillan V, Rogowski D. Postoperative pain therapy: a survey of patients' expectations and their experiences. Pain. 1990;41(3):303-307. http://www.ncbi.nlm.nih.gov/pubmed/1697058. Accessed January 5, 2019.

75. Sjöling M, Nordahl G, Olofsson N, Asplund K. The impact of preoperative information on state anxiety, postoperative pain and satisfaction with pain management. Patient Educ Couns. 2003;51(2):169-176. http://www.ncbi.nlm.nih.gov/pubmed/14572947. Accessed January 5, 2019.

76. Stewart MA. Effective physician-patient communication and health outcomes: a review. CMAJ. 1995;152(9):1423-1433. http://www.ncbi.nlm.nih.gov/pubmed/7728691. Accessed January 5, 2019.

77. Egbert LD, Battit GE, Welch CE, Bartlett MK. Reduction of Postoperative Pain by Encouragement and Instruction of Patients. N Engl J Med. 1964;270(16):825-827. doi:10.1056/NEJM196404162701606

78. Langer EJ, Janis IL, Wolfer JA. Reduction of psychological stress in surgical patients. $J$ Exp Soc Psychol. 1975;11(2):155-165. doi:10.1016/S0022-1031(75)80018-7

79. Niemi-Murola L, Pöyhiä R, Onkinen K, Rhen B, Mäkelä A, Niemi TT. Patient satisfaction with postoperative pain management--effect of preoperative factors. Pain Manag Nurs. 2007;8(3):122-129. doi:10.1016/j.pmn.2007.05.003

80. Jamison RN, Ross MJ, Hoopman P, et al. Assessment of postoperative pain management: patient satisfaction and perceived helpfulness. Clin J Pain. 1997;13(3):229-236. http://www.ncbi.nlm.nih.gov/pubmed/9303255. Accessed January 5, 2019.

81. Gammon J, Mulholland CW. Effect of preparatory information prior to elective total hip replacement on psychological coping outcomes. J Adv Nurs. 1996;24(2):303-308. http://www.ncbi.nlm.nih.gov/pubmed/8858434. Accessed January 5, 2019. 
82. Andersson, Viveka, Eva Otterstrom-Rydberg, and Ann-Kristin Karlsson. "The importance of written and verbal information on pain treatment for patients undergoing surgical interventions." Pain Management Nursing 16.5 (2015): 634-641.

83. Johnson, Anne, Jayne Sandford, and Jessica Tyndall. "Written and verbal information versus verbal information only for patients being discharged from acute hospital settings to home." Cochrane Database of Systematic Reviews 4 (2003).

84. Derry S, Derry CJ, Moore RA. Single dose oral ibuprofen plus oxycodone for acute postoperative pain in adults. Cochrane Database Syst Rev. June 2013. doi:10.1002/14651858.CD010289.pub2

85. Hill M V., Stucke RS, McMahon ML, Beeman JL, Barth RJ. An Educational Intervention Decreases Opioid Prescribing After General Surgical Operations. Ann Surg. 2018;267(3):468-472. doi:10.1097/SLA.0000000000002198

86. Howard R, Waljee J, Brummett C, Englesbe M, Lee J. Reduction in Opioid Prescribing Through Evidence-Based Prescribing Guidelines. JAMA Surg. 2018;153(3):285. doi:10.1001/jamasurg.2017.4436

87. Sekhri S, Arora NS, Cottrell H, et al. Probability of Opioid Prescription Refilling After Surgery. Ann Surg. 2018;268(2):271-276. doi:10.1097/SLA.0000000000002308

88. Zivin K, White JO, Chao S, et al. Implementing Electronic Health Record Default Settings to Reduce Opioid Overprescribing: A Pilot Study. Pain Med. January 2018. doi:10.1093/pm/pnx304

89. Malte CA, Berger D, Saxon AJ, et al. Electronic Medical Record Alert Associated With Reduced Opioid and Benzodiazepine Coprescribing in High-risk Veteran Patients. Med Care. 2018;56(2):171-178. doi:10.1097/MLR.0000000000000861

90. Luk LJ, Mosen D, MacArthur CJ, Grosz AH. Implementation of a Pediatric Posttonsillectomy Pain Protocol in a Large Group Practice. Otolaryngol Neck Surg. 2016;154(4):720-724. doi:10.1177/0194599815627810

91. Benyamin R, Trescot AM, Datta S, et al. Opioid complications and side effects. Pain Physician. 2008;11(2 Suppl):S105-20. http://www.ncbi.nlm.nih.gov/pubmed/18443635. Accessed January 5, 2019.

92. White PF. The Role of Non-Opioid Analgesic Techniques in the Management of Pain After Ambulatory Surgery. Anesth Analg. 2002;94(3):577-585. doi:10.1097/00000539200203000-00019

93. Barker JC, DiBartola K, Wee C, et al. Preoperative Multimodal Analgesia Decreases Postanesthesia Care Unit Narcotic Use and Pain Scores in Outpatient Breast Surgery. Plast Reconstr Surg. 2018;142(4):443e-450e. doi:10.1097/PRS.0000000000004804 
94. Lee JH. Anesthesia for ambulatory surgery. Korean J Anesthesiol. 2017;70(4):398-406. doi:10.4097/kjae.2017.70.4.398

95. Elvir-Lazo OL, White PF. The role of multimodal analgesia in pain management after ambulatory surgery. Curr Opin Anaesthesiol. 2010;23(6):697-703.

doi:10.1097/ACO.0b013e32833fad0a

96. Quality Ontario H. Opioid Prescribing Guidelines for Acute Pain. https://www.hqontario.ca/portals/0/documents/evidence/quality-standards/qs-opioidacute-pain-quality-statements-in-brief-en.pdf. Accessed January 5, 2019.

97. Ong CK-S, Lirk P, Seymour RA, Jenkins BJ. The Efficacy of Preemptive Analgesia for Acute Postoperative Pain Management: A Meta-Analysis. Anesth Analg. 2005;100(3):757-773. doi:10.1213/01.ANE.0000144428.98767.0E

98. Warltier DC, Ph D, Møiniche S, et al. A Qualitative and Quantitative Systematic Review of Preemptive Analgesia for Postoperative Pain Relief. 2018;(4).

99. Khaira HS, Wolf JS. Intraoperative local anesthesia decreases postoperative parenteral opioid requirements for transperitoneal laparoscopic renal and adrenal surgery: a randomized, double-blind, placebo controlled investigation. J Urol. 2004;172(4 Pt 1):1422-1426. http://www.ncbi.nlm.nih.gov/pubmed/15371860. Accessed January 7 , 2019.

100. Fassoulaki A, Triga A, Melemeni A, Sarantopoulos C. Multimodal Analgesia with Gabapentin and Local Anesthetics Prevents Acute and Chronic Pain After Breast Surgery for Cancer. Anesth Analg. 2005;101(5):1427-1432.

doi:10.1213/01.ANE.0000180200.11626.8E

101. Fassoulaki A, Triga A, Melemeni A, Sarantopoulos C. Multimodal Analgesia with Gabapentin and Local Anesthetics Prevents Acute and Chronic Pain After Breast Surgery for Cancer. Anesth Analg. 2005;101(5):1427-1432.

doi:10.1213/01.ANE.0000180200.11626.8E

102. Stuhldreher JM, Adamina M, Konopacka A, Brady K, Delaney CP. Effect of local anesthetics on postoperative pain and opioid consumption in laparoscopic colorectal surgery. Surg Endosc. 2012;26(6):1617-1623. doi:10.1007/s00464-011-2079-1

103. Rosaeg OP, Bell M, Cicutti NJ, Dennehy KC, Lui ACP, Krepski B. Pre-incision infiltration with lidocaine reduces pain and opioid consumption after reduction mammoplasty. Reg Anesth Pain Med. 1998;23(6):575-579. doi:10.1016/S10987339(98)90084-0

104. Harrison CA, Morris S, Harvey JS. Effect of ilioinguinal and iliohypogastric nerve block and wound infiltration with $0.5 \%$ bupivacaine on postoperative pain after hernia repair. $\mathrm{Br}$ 
J Anaesth. 1994;72(6):691-693. http://www.ncbi.nlm.nih.gov/pubmed/8024918. Accessed January 10, 2019.

105. Chahar P, Cummings KC, III. Liposomal bupivacaine: a review of a new bupivacaine formulation. J Pain Res. 2012;5:257-264. doi:10.2147/JPR.S27894

106. Dasta J, Ramamoorthy S, Patou G, Sinatra R. Bupivacaine liposome injectable suspension compared with bupivacaine $\mathrm{HCl}$ for the reduction of opioid burden in the postsurgical setting. Curr Med Res Opin. 2012;28(10):1609-1615. doi:10.1185/03007995.2012.721760

107. Kumar K, Kirksey MA, Duong S, Wu CL. A Review of Opioid-Sparing Modalities in Perioperative Pain Management. Anesth Analg. 2017;125(5):1749-1760. doi:10.1213/ANE.0000000000002497

108. Kairaluoma PM, Bachmann MS, Korpinen AK, Rosenberg PH, Pere PJ. Single-Injection Paravertebral Block Before General Anesthesia Enhances Analgesia After Breast Cancer Surgery With and Without Associated Lymph Node Biopsy. Anesth Analg. December 2004:1837-1843. doi:10.1213/01.ANE.0000136775.15566.87

109. Shim S-M, Park J-H, Hyun D-M, Jeong E-K, Kim S-S, Lee H-M. The effects of adjuvant intrathecal fentanyl on postoperative pain and rebound pain for anorectal surgery under saddle anesthesia. Korean J Anesthesiol. 2018;71(3):213-219. doi:10.4097/kja.d.18.27097

110. Karmakar MK, Samy W, Li JW, et al. Thoracic Paravertebral Block and Its Effects on Chronic Pain and Health-Related Quality of Life After Modified Radical Mastectomy. Reg Anesth Pain Med. 2014;39(4):289-298. doi:10.1097/AAP.0000000000000113

111. Correll, Darin J; Viscusi, Eugene R; Grunwald, Zvi; Moore JHJ. Epidural Analgesia Compared With Intravenous Morphine Patient-Controlled Analgesia: Postoperative Outcome Measures After Mastectomy With Immediate TRAM Flap Breast Reconstruction. Reg Anesth Pain Med. 2001;26(5):444-449.

112. Naja MZ, Ziade MF, El Rajab M. Sacrococcygeal local anaesthesia versus general anaesthesia for pilonidal sinus surgery: a prospective randomised trial. Anaesthesia. 2003;58(10):1007-1012. doi:10.1046/j.1365-2044.2003.03401.x

113. Fletcher D, Zetlaoui P, Monin S, Bombart M, Samii K. Influence of timing on the analgesic effect of intravenous ketorolac after orthopedic surgery. Pain. 1995;61(2):291297. doi:10.1016/0304-3959(94)00184-G

114. Gillberg LE, Harsten AS, Ståhl LB. Preoperative diclofenac sodium reduces postlaparoscopy pain. Can J Anaesth. 1993;40(5):406-408. doi:10.1007/BF03009507

115. De Oliveira GS, Agarwal D, Benzon HT. Perioperative Single Dose Ketorolac to Prevent Postoperative Pain. Anesth Analg. 2012;114(2):424-433.

doi:10.1213/ANE.0b013e3182334d68 
116. Waldron NH, Jones CA, Gan TJ, Allen TK, Habib AS. Impact of perioperative dexamethasone on postoperative analgesia and side-effects: systematic review and metaanalysis. Br J Anaesth. 2013;110(2):191-200. doi:10.1093/bja/aes431

117. De Oliveira GS, Almeida MD, Benzon HT, McCarthy RJ. Perioperative Single Dose Systemic Dexamethasone for Postoperative Pain. Anesthesiology. 2011;115(3):575-588. doi:10.1097/ALN.0b013e31822a24c2

118. Gärtner R, Kroman N, Callesen T, Kehlet H. Multimodal prevention of pain, nausea and vomiting after breast cancer surgery. Minerva Anestesiol. 2010;76(10):805-813. http://www.ncbi.nlm.nih.gov/pubmed/20935616. Accessed January 7, 2019.

119. Gómez-Hernández J, Orozco-Alatorre AL, Domínguez-Contreras M, et al. Preoperative dexamethasone reduces postoperative pain, nausea and vomiting following mastectomy for breast cancer. BMC Cancer. 2010;10(1):692. doi:10.1186/1471-2407-10-692

120. Coloma M, Duffy LL, White PF, Kendall Tongier W, Huber PJ. Dexamethasone facilitates discharge after outpatient anorectal surgery. Anesth Analg. 2001;92(1):85-88. http://www.ncbi.nlm.nih.gov/pubmed/11133606. Accessed January 7, 2019.

121. Ræder JC, Steine S, Vatsgar TT. Oral Ibuprofen Versus Paracetamol Plus Codeine for Analgesia After Ambulatory Surgery. Anesth Analg. 2001;92(6):1470-1472. doi:10.1097/00000539-200106000-00023

123. Stessel B, Theunissen M, Fiddelers AA, et al. Controlled-release oxycodone versus naproxen at home after ambulatory surgery: a randomized controlled trial. Curr Ther Res Clin Exp. 2014;76:120-125. doi:10.1016/j.curtheres.2014.10.001

124. Rainsford K. Profile and mechanisms of gastrointestinal and other side effects of nonsteroidal anti-inflammatory drugs (NSAIDs). Am J Med. 1999;107(6):27-35. doi:10.1016/S0002-9343(99)00365-4

125. Møiniche S, Rømsing J, Dahl JB, Tramèr MR. Nonsteroidal antiinflammatory drugs and the risk of operative site bleeding after tonsillectomy: a quantitative systematic review. Anesth Analg. 2003;96(1):68-77, table of contents. http://www.ncbi.nlm.nih.gov/pubmed/12505926. Accessed January 10, 2019.

126. Van Hecken, A., Schwartz, J. I., Depré, M., De Lepeleire, I., Dallob, A., Tanaka, W., ... \& Ebel, D. L. (2000). Comparative inhibitory activity of rofecoxib, meloxicam, diclofenac, ibuprofen, and naproxen on COX-2 versus COX-1 in healthy volunteers. The Journal of Clinical Pharmacology, 40(10), 1109-1120.

127. Meloxicam. In Lexicomp Online Database: Lexicomp Inc. Available at: https://online.lexi.com/lco/medguides/615074pd. Accessed Sept 2, 2019 
128. Schoenfeld, P. (1999). Gastrointestinal safety profile of meloxicam: a meta-analysis and systematic review of randomized controlled trials. The American journal of medicine, 107(6), 48-54.

129. Ong CKS, Seymour RA, Lirk P, Merry AF. Combining Paracetamol (Acetaminophen) with Nonsteroidal Antiinflammatory Drugs: A Qualitative Systematic Review of Analgesic Efficacy for Acute Postoperative Pain. Anesth Analg. February 2010:1. doi:10.1213/ANE.0b013e3181cf9281

130. Toms L, McQuay HJ, Derry S, Moore RA. Single dose oral paracetamol (acetaminophen) for postoperative pain in adults. Cochrane database Syst Rev. 2008;(4):CD004602. doi:10.1002/14651858.CD004602.pub2

131. McNicol ED, Ferguson MC, Haroutounian S, Carr DB, Schumann R. Single dose intravenous paracetamol or intravenous propacetamol for postoperative pain. Cochrane Database Syst Rev. May 2016. doi:10.1002/14651858.CD007126.pub3

132. Derry CJ, Derry S, Moore RA. Single dose oral ibuprofen plus paracetamol (acetaminophen) for acute postoperative pain. Cochrane Database Syst Rev. 2013;(6):CD010210. doi:10.1002/14651858.CD010210.pub2

133. Kumar K, Kirksey MA, Duong S, Wu CL. A Review of Opioid-Sparing Modalities in Perioperative Pain Management. Anesth Analg. 2017;125(5):1749-1760. doi:10.1213/ANE.0000000000002497

134. Jouguelet-Lacoste J, La Colla L, Schilling D, Chelly JE. The Use of Intravenous Infusion or Single Dose of Low-Dose Ketamine for Postoperative Analgesia: A Review of the Current Literature. Pain Med. 2015;16(2):383-403. doi:10.1111/pme.12619

135. Sabine Himmelseher, Marcel E. Durieux. Ketamine for Perioperative Pain Management. Anesthesiol J Am Soc Anesthesiol. 2005;102(1):211-220.

http://anesthesiology.pubs.asahq.org/article.aspx?articleid=2026055. Accessed January 9, 2019.

136. Radvansky BM, Shah K, Parikh A, Sifonios AN, Le V, Eloy JD. Role of Ketamine in Acute Postoperative Pain Management: A Narrative Review. Biomed Res Int. 2015;2015:1-10. doi:10.1155/2015/749837

137. Jessen Lundorf L, Korvenius Nedergaard H, Møller AM. Perioperative dexmedetomidine for acute pain after abdominal surgery in adults. Cochrane Database Syst Rev. February 2016. doi:10.1002/14651858.CD010358.pub2

138. Blaudszun G, Lysakowski C, Elia N, Tramèr MR. Effect of Perioperative Systemic $\alpha 2$ 
Agonists on Postoperative Morphine Consumption and Pain Intensity. Anesthesiology. 2012;116(6):1312-1322. doi:10.1097/ALN.0b013e31825681cb

139. Behdad S, Ayatollahi V, Yazdi AG, Mortazavizadeh A, Niknam F. Effect of oral low dose clonidine premedication on postoperative pain in patients undergoing abdominal hysterectomy: a randomized placebo controlled clinical trial. Rev Med Chir Soc Med Nat Iasi. 117(4):934-941. http://www.ncbi.nlm.nih.gov/pubmed/24502072. Accessed January $10,2019$.

140. Yazicioğlu D, Caparlar C, Akkaya T, Mercan U, Kulaçoğlu H. Tizanidine for the management of acute postoperative pain after inguinal hernia repair. Eur J Anaesthesiol. 2016;33(3):215-222. doi:10.1097/EJA.0000000000000371

141. Clarke H, Poon M, Weinrib A, Katznelson R, Wentlandt K, Katz J. Preventive Analgesia and Novel Strategies for the Prevention of Chronic Post-Surgical Pain. 2015. doi:10.1007/s40265-015-0365-2

142. Hah J, Mackey SC, Schmidt P, et al. Effect of Perioperative Gabapentin on Postoperative Pain Resolution and Opioid Cessation in a Mixed Surgical Cohort. JAMA Surg. 2018;153(4):303. doi:10.1001/jamasurg.2017.4915

143. Hurley RW, Cohen SP, Williams KA, Rowlingson AJ, Wu CL. The Analgesic Effects of Perioperative Gabapentin on Postoperative Pain: A Meta-Analysis. Reg Anesth Pain Med. 2006;31(3):237-247. doi:10.1016/J.RAPM.2006.01.005

144. Peng PW, Wijeysundera DN, Li CC. Use of gabapentin for perioperative pain control -- a meta-analysis. Pain Res Manag. 2007;12(2):85-92. http://www.ncbi.nlm.nih.gov/pubmed/17505569. Accessed January 10, 2019.

145. Doleman B, Heinink TP, Read DJ, Faleiro RJ, Lund JN, Williams JP. A systematic review and meta-regression analysis of prophylactic gabapentin for postoperative pain. Anaesthesia. 2015;70(10):1186-1204. doi:10.1111/anae.13179

146. Fabritius ML, Geisler A, Petersen PL, et al. Gabapentin for post-operative pain management - a systematic review with meta-analyses and trial sequential analyses. Acta Anaesthesiol Scand. 2016;60(9):1188-1208. doi:10.1111/aas.12766

147. Eipe N, Penning J, Yazdi F, et al. Perioperative use of pregabalin for acute pain - a systematic review and meta-analysis. Pain. 2015;156(7):1284-1300. doi:10.1097/j.pain.0000000000000173

148. Mishriky BM, Waldron NH, Habib AS. Impact of pregabalin on acute and persistent postoperative pain: a systematic review and meta-analysis. Br J Anaesth. 2015;114(1):1031. doi:10.1093/bja/aeu293

149. Lam DMH, Choi S-W, Wong SSC, Irwin MG, Cheung C-W. Efficacy of Pregabalin in 
Acute Postoperative Pain Under Different Surgical Categories. Medicine (Baltimore). 2015;94(46):e1944. doi:10.1097/MD.0000000000001944

150. Ho K-Y, Tay W, Yeo M-C, et al. Duloxetine reduces morphine requirements after knee replacement surgery. Br J Anaesth. 2010;105(3):371-376. doi:10.1093/bja/aeq158

151. Attia JZ, Mansour HS. Perioperative Duloxetine and Etoricoxibto improve postoperative pain after lumbar Laminectomy: a randomized, double-blind, controlled study. BMC Anesthesiol. 2017;17(1):162. doi:10.1186/s12871-017-0450-z

152. Castro-Alves LJ, Oliveira de Medeiros ACP, Neves SP, et al. Perioperative Duloxetine to Improve Postoperative Recovery After Abdominal Hysterectomy. Anesth Analg. 2016;122(1):98-104. doi:10.1213/ANE.0000000000000971

153. Levine JD, Gordon NC, Smith R, McBryde R. Desipramine enhances opiate postoperative analgesia. Pain. 1986;27(1):45-49. http://www.ncbi.nlm.nih.gov/pubmed/3785963. Accessed January 10, 2019.

154. Vahedi P, Salehpour F, Aghamohammadi D, et al. Single Dose Preemptive Amitriptyline Reduces Postoperative Neuropathic Pain After Lumbar Laminectomy and Discectomy. Neurosurg Q. 2010;20(3):151-158. doi:10.1097/WNQ.0b013e3181ebce15

155. Kerrick JM, Fine PG, Lipman AG, Love G. Low-dose amitriptyline as an adjunct to opioids for postoperative orthopedic pain: a placebo-controlled trial. Pain. 1993;52(3):325-330. http://www.ncbi.nlm.nih.gov/pubmed/8460050. Accessed January $10,2019$.

156. Schnabel A, Pogatzki-Zahn E. Predictors of chronic pain following surgery. What do we know? Der Schmerz. 2010;24(5):517-533. doi:10.1007/s00482-010-0932-0

157. Clarke H, Poon M, Weinrib A, Katznelson R, Wentlandt K, Katz J. Preventive Analgesia and Novel Strategies for the Prevention of Chronic Post-Surgical Pain. Drugs. 2015;75(4):339-351. doi:10.1007/s40265-015-0365-2

158. Reddi D. Preventing chronic postoperative pain. Anaesthesia. 2016;71:64-71. doi:10.1111/anae.13306

159. Koc, M., Tez, M., Yoldaş, Ö., Dizen, H., \& Göçmen, E. Cooling for the reduction of postoperative pain: prospective randomized study. Hernia, 2006; 10(2), 184-186.

160. Watkins, A. A., Johnson, T. V., Shrewsberry, A. B., Nourparvar, P., Madni, T., Watkins, C. J., ... \& Master, V. A. (2014). Ice packs reduce postoperative midline incision pain and narcotic use: a randomized controlled trial. Journal of the American College of Surgeons, (2014); 219(3), 511-517. 
161. Ljungqvist O, Scott M, Fearon K,. "Enhanced recovery after surgery: a review." JAMA surgery 152.3 (2017): 292-298.

162. Parrish AB, O'Neill SM, Crain SR, et al. An Enhanced Recovery After Surgery (ERAS) Protocol for Ambulatory Anorectal Surgery Reduced Postoperative Pain and Unplanned Returns to Care After Discharge. World J Surg. 2018;42(7):1929-1938. doi:10.1007/s00268-017-4414-8

163. Offodile AC, Gu C, Boukovalas S, et al. Enhanced recovery after surgery (ERAS) pathways in breast reconstruction: systematic review and meta-analysis of the literature. Breast Cancer Res Treat. October 2018:1-13. doi:10.1007/s10549-018-4991-8

164. Rojas KE, Manasseh D-M, Flom PL, et al. A pilot study of a breast surgery Enhanced Recovery After Surgery (ERAS) protocol to eliminate narcotic prescription at discharge. Breast Cancer Res Treat. 2018;171(3):621-626. doi:10.1007/s10549-018-4859-y

165. Chiu C, Aleshi P, Esserman LJ, et al. Improved analgesia and reduced post-operative nausea and vomiting after implementation of an enhanced recovery after surgery (ERAS) pathway for total mastectomy. BMC Anesthesiol. 2018;18(1):41. doi:10.1186/s12871-0180505-9

166. Singh S, Clarke C, Lawendy AR, Macleod M, Sanders D, Tieszer C. A prospective, randomized controlled trial of the impact of written discharge instructions for postoperative opioids on patient pain satisfaction and on minimizing opioid risk exposure in orthopaedic surgery. Curr Orthop Pract. 2018;29(4):292-296. doi:10.1097/BCO.0000000000000632 


\subsection{CHAPTER 2. THE STANDARDIZATION OF OUTPATIENT PROCEDURE (STOP) NARCOTICS AFTER ANORECTAL SURGERY: A PROSPECTIVE NON-INFERIORITY STUDY TO REDUCE OPIOID USE}

Hartford LB, Murphy PB, Gray DK, Maciver AH, Clarke FM, Allen LJ, Garcia-Ochoa C, Leslie $K A$, Van Koughnett JAM.

\subsection{BACKGROUND:}

Prescription opioid abuse is a major public health concern in North America, rising to one of the leading causes of injury-related deaths. ${ }^{1,2}$ Surgeons play a crucial role in the opioid crisis. While good pain control after surgery is important, post-operative prescribing of opioids after outpatient surgery is often excessive, and in many cases only a fraction of the prescribed opioids are required for adequate pain control, including after anorectal surgery. ${ }^{3-5}$

Analgesia is an important aspect of surgical recovery; however, patients who are prescribed opioids are more likely to become chronic opioid users. ${ }^{6}$ Every refill and additional week of opioid use is associated with a large increase in opioid abuse in opioid naïve patients. ${ }^{7}$ In addition, diversion of unused prescribed medication is a significant source of abused opioids. ${ }^{8}$

Anorectal surgery often requires a general anesthetic for relaxation and procedural pain control, but usually does not require admission to hospital. ${ }^{9}$ There are no published evidence-based guidelines that specifically address the appropriate amount of opioid pills to prescribe after outpatient anorectal procedures. ${ }^{10-12}$ However, various interventions including various oral medications (non-steroidal anti-inflammatory [NSAID], gabapentin, metronidazole, etc.), topical anesthetics, nerve blocks and intrathecal injections, and suppositories have been studied. ${ }^{13-21}$ The PROSPECT update on pain control after hemorrhoidectomy acknowledged that most recommendations are based on single-intervention smaller studies, and highlighted the importance of a multi-modality approach. ${ }^{22}$

The Standardization of Outpatient Procedure Narcotics (STOP Narcotics) quality improvement initiative was developed at London Health Sciences Centre (LHSC) after identifying Opioid 
Prescribing for Acute Pain as a priority area of focus based on the Joint Statement of Action to Address the Opioid Crisis, issued by Health Canada in $2017 .{ }^{23}$ We created a multi-pronged strategy to standardize post-operative pain management and reduce opioid prescriptions, while adequately controlling pain after outpatient surgery. The positive impact of the STOP Narcotics initiative after outpatient laparoscopic cholecystectomy and open hernia repairs was recently published. ${ }^{24}$

This study is a prospective non-inferiority evaluation of the efficacy of the STOP Narcotics initiative in patients undergoing outpatient anorectal surgery, including post-operative pain control, patient satisfaction, and compliance. We hypothesized that the STOP Narcotics protocol would be associated with non-inferior pain control while reducing opioid prescriptions by surgeons and opioid use among patients undergoing outpatient anorectal surgery.

\subsection{METHODS:}

\subsubsection{Study Design and Setting}

A non-inferiority, prospective pre- and post-intervention study was conducted from July 2017 to April 2018. Surgeries were performed at LHSC and St. Joseph's Health Care Centre, encompassing 3 academic hospitals where a high volume of outpatient general and colorectal surgery is performed in London, Ontario, Canada. The STrengthening the Reporting of OBservation studies in Epidemiology (STROBE) guidelines were followed. ${ }^{25}$ Ethics approval was obtained through the Health Sciences Research Ethics Board at Western University (HSREB\# 109651). 


\subsubsection{Pre- and Post-Intervention Groups}

To evaluate the efficacy of the STOP Narcotics initiative, adult patients aged 18 to 75 years, undergoing outpatient anorectal surgery, requiring an anesthetic in the operating room, were included in the study. Surgeries included examination under anesthesia (EUA), fistulotomy, seton placement, rectal advancement flap, anal dilation, transanal excision (excluding transanal endoscopic surgery), abscess drainage, hemorrhoidectomy and pilonidal sinus excision. Patients were excluded if they were known regular opioid users or had chronic pain conditions (excluding osteoarthritis and back pain), chronic kidney disease, or active peptic ulcer disease. Once patients were enrolled after an initial operation, any subsequent procedures were excluded to avoid duplication.

A control group (pre-intervention) from July 17, 2017 to October 18, 2017 was compared to the post-intervention group from October 23, 2017 to April 30, 2018. The control group received education at the discretion of the surgeon and in the form of a written sheet, which did not address pain expectations or medication utilization instructions. The management of patients in the post-intervention group only differed by exposure to the STOP Narcotics intervention protocol.

\subsubsection{Intervention}

The STOP Narcotics initiative involved the implementation of a 4-pronged strategy: patient education through counseling and written materials; health care provider education (surgeons, anaesthetists, residents, and nurses); intra-operative multi-modal analgesia; and post-operative multi-modal opioid reduction strategies for pain control. The protocol was designed with input from relevant stakeholders (surgeons, anesthetists, nurses, and patients) and was instituted across the Division of General Surgery at Western University. The division is comprised of 20 surgeons who perform outpatient general surgical procedures, including 10 surgeons who performed anorectal surgeries during our study period. 


\subsubsection{Patient Education}

Patient education included four areas of focus. Verbal education for patients about pain expectations and discomfort after surgery was coupled with instructions for optimal utilization of multi-modal analgesia medications. This discussion was initiated at the time of consent for the procedure and reinforced on the day of surgery. Patients were given instructions on the appropriate use of opioids after surgery. A limited opioid prescription (10 tablets) was provided as a separate prescription with an expiry date of 7 days and was to be filled only if the other pain control strategies were deemed ineffective by the patient. Written instructions were given to sit in warm water 2 to 3 times per day for comfort. Healthy toileting habits, maintenance of adequate hydration, use of high fiber diets and fiber supplements, and optimal use of the prescribed laxative and other over the counter laxatives were also described to the patient to avoid difficult bowel movements. Patients were also educated on the appropriate disposal of unused medication. Instructions were reiterated on the day of operation by the surgical team and reviewed prior to leaving the hospital with standardized education sheets and verbal reinforcement by nursing staff.

\subsubsection{Provider Education}

Providers, including surgeons, anaesthetists, residents, nurses and office staff, were educated in large group formats (divisional rounds and meetings), written posters in clinic areas, electronically via email, and verbally to individuals. Education focused on the need for opioid reduction strategies, understanding the multi-modal analgesia initiative, and supporting patient and caregiver pain control expectations after surgery.

\subsubsection{Intra-Operative Pain Management Strategy}

As part of the surgical safety checklist prior to induction of anesthesia, the surgical team requested the provision of intravenous ketorolac (15-30 mg IV), ondansetron (4-8 mg IV), and dexamethasone (4-8 $\mathrm{mg} \mathrm{IV}$ ), to be administered by the anesthetist during the operation. The final 
decision on intra-operative medications to be administered was left to the discretion of the anesthesia team.

\subsubsection{Post-Operative Pain Management Strategy}

A prescription for an NSAID (meloxicam $7.5 \mathrm{mg}$ or naproxen $400 \mathrm{mg}$ tablets) was given with instructions to use it twice a day for 72 hours. Patients also received instructions for regular use of acetaminophen $500 \mathrm{mg}$ tablet every 6 hours for the first 72 hours. After 72 hours, patients were instructed to use ibuprofen $400 \mathrm{mg}$ and acetaminophen $500 \mathrm{mg}$ as needed for pain control. A separate prescription for 10 tablets of tramadol $50 \mathrm{mg}$ or codeine $30 \mathrm{mg}$ with an expiry date of 7 days after surgery was provided, with specific instructions to fill this prescription only if adequate pain control was not achieved by the other strategies provided. Patients were also given a prescription for metronidazole $500 \mathrm{mg}$ ( 1 tablet by mouth every 12 hours for 5 days) and polyethylene glycol (PEG) $17 \mathrm{~g}$ powder to be taken once to twice daily to avoid constipation and until stool was consistently soft (Appendix 5.2). Standardized educational materials and instructions on pain control strategies were distributed to patients in the recovery room, prior to discharge. Other post-operative instructions such as activity levels, wound care, and return to work were provided at the discretion of the surgeon.

\subsubsection{Outcomes}

The primary outcome was patient-reported average pain level in the first 7 post-operative days, measured using a modified Brief Pain Inventory Survey at their first post-operative clinic appointment (typically at 4 weeks post-operatively). ${ }^{26,27}$ Secondary outcomes included overall quality of pain management after surgery and patient function in the first post-operative week. The clinic survey also captured patient reported anti-inflammatory and acetaminophen use, filling of the opioid prescription, percentage of opioid tablets used, number of prescription refills, and medication disposal to a pharmacy or the outpatient clinic. In addition to self-reported outcomes, medical records were reviewed to identify prescriptions (all are processed and tracked via the electronic medical record) and post-operative complications. Oral morphine equivalents (OME) were calculated to quantify opioids taken (Table 2.1). 
Table 2.1 Oral Morphine Equivalents (OME)

\begin{tabular}{lc}
\hline & Oral Morphine Equivalents \\
& \\
\hline \multicolumn{10}{l}{$\mathbf{1 0}$ tablets } & OME \\
\hline Morphine $5 \mathrm{mg}$ & 50 \\
\hline Oxycodone $5 \mathrm{mg}$ & 75 \\
\hline Codeine $30 \mathrm{mg}$ & 45 \\
\hline Tramadol $50 \mathrm{mg}$ & 50 \\
\hline Hydromorphone 2 mg & 80 \\
\hline
\end{tabular}

Oral Morphine Equivalents for 10 tablets of common surgical prescriptions.

Agency Medical Directors' Group. ${ }^{27}$ http://www.agencymeddirectors.wa.gov/calculator/dosecalculator.htm

\subsubsection{Statistical Analysis}

The sample size was calculated based on a meaningful clinical difference (MCD) of two points on the numerical pain rating scale for the primary outcome. ${ }^{27,28}$ Using an alpha of 0.025 , power of $90 \%$, and a non-inferiority limit of two MCD points on the numeric rating scale, 44 patients in each of the pre- and post-intervention groups were required.

All statistical analyses were performed using SPSS Version 24 (IBM Corp., SAS Institute, Cary, North Carolina, USA). Continuous variables were expressed as medians with interquartile ranges for non-normally distributed variables, and as means and standard deviations for normally distributed variables. Mann-Whitney $\mathrm{U}$ tests and independent samples t-tests were used to assess for differences between groups for medians and means, respectively. Chi-square tests and Fischer's Exact tests were performed for categorical data. Non-inferiority was tested using the two-sample, equal variance, t-test for mean difference. The primary outcome was tested for noninferiority with a one-sided test. Two-sided tests were used for secondary variables. A p-value of $<0.025$ for the primary outcome was considered statistically significant and $\mathrm{p}<0.05$ for secondary variables. 


\subsection{RESULTS:}

\subsubsection{Study Population}

The study population consisted of 115 patients. In addition to the specified exclusion criteria, 10 additional patients were excluded (Figure 2.1). After exclusion, there were 42 patients in the preintervention group and 51 patients in the post-intervention groups. Pre- and post-intervention groups were similar in age and sex (Table 2.2).

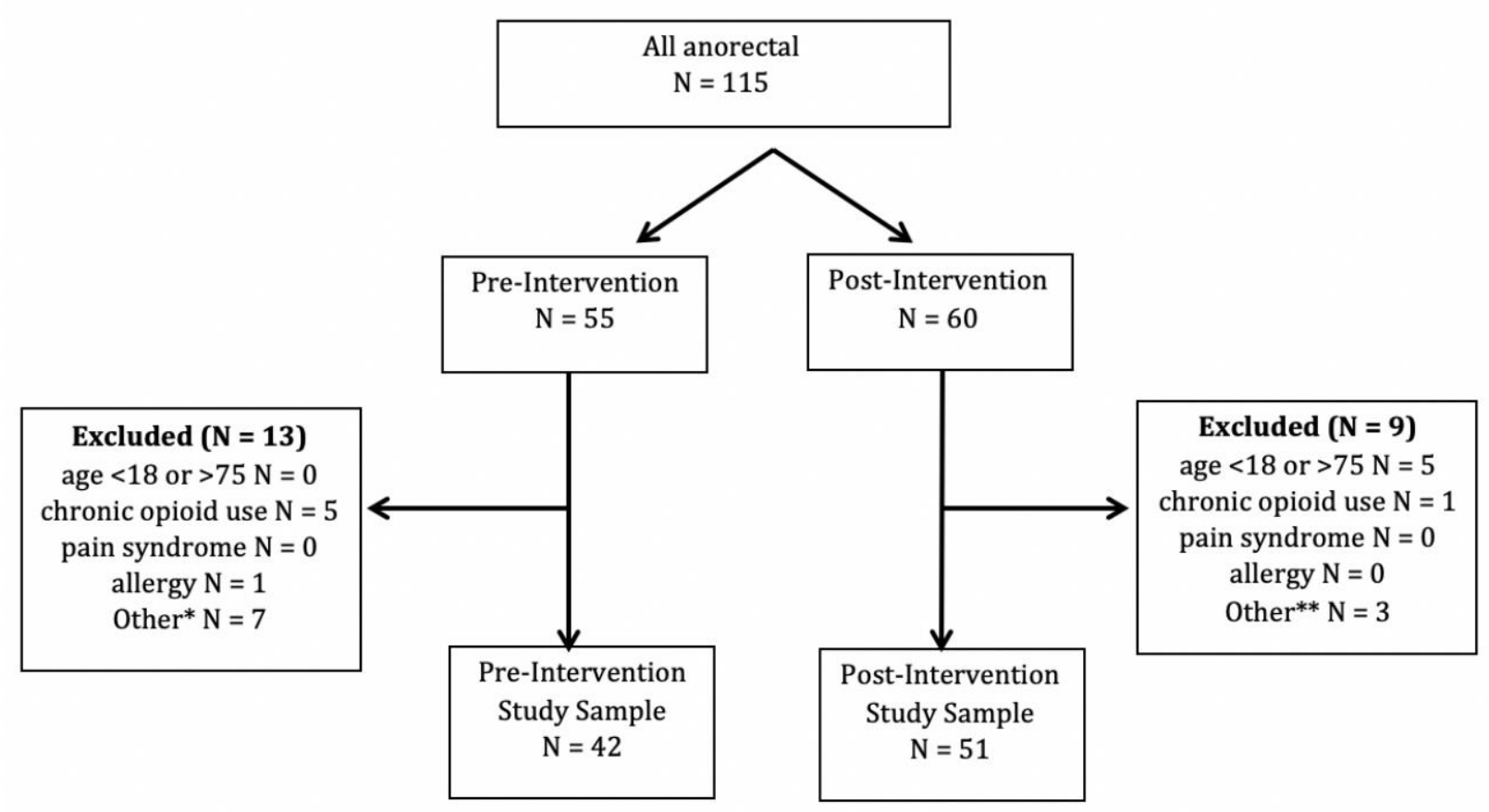

Figure 2.1 Flow chart of included patients. * $\mathrm{N}=2$ (did not complete the brief pain inventory), $\mathrm{N}$ $=5$ (duplicates); $* * \mathrm{~N}=3$ (duplicates) 
Table 2.2 Patient Demographics

\begin{tabular}{lccl}
\hline Patient Demographics & $\begin{array}{c}\text { Pre- } \\
\text { intervention }\end{array}$ & $\begin{array}{c}\text { Post-intervention } \\
\text { N = 42 }\end{array}$ & p-value \\
& $\mathbf{N}=\mathbf{5 1}$ & \\
\hline Fistulotomy n (\%) & $5(35.7)$ & $22(43.1)$ & \\
Hemorrhoidectomy n (\%) & $6(14.3)$ & $8(15.7)$ & \\
Excision of anal lesion n (\%) & $4(9.5)$ & $4(7.8)$ & \\
\hline Seton placement n (\%) & $4(9.5)$ & $5(9.8)$ & \\
\hline Anal dilation n (\%) & $1(2.4)$ & $5(9.8)$ & \\
Pilonidal sinus excision n (\%) & $1(2.4)$ & $2(5.9)$ & \\
\hline Rectal advancement flap n (\%) & $2(4.8)$ & $1(2.0)$ & \\
\hline Excision of rectal lesion n (\%) & $2(4.8)$ & $1(2.0)$ & \\
Abscess drainage n (\%) & $2(4.8)$ & $0(0.0)$ & \\
\hline EUA n (\%) & $45(16)$ & $44(14)$ & 0.67 \\
\hline Age in years, mean (SD) & $27(64)$ & $26(51)$ & 0.20 \\
\hline Male sex, n (\%) & & &
\end{tabular}

EUA, Examination under anesthesia; SD, standard deviation

* Study population after exclusion

\subsubsection{Patient-Reported Quality Outcomes}

The primary outcome of average post-operative pain score in the first 7 post-operative days was non-inferior in the post-intervention group compared to pre-intervention group ( 2.8 vs. 2.6/10, $\mathrm{p}$ $=0.33$ ). The reported quality of pain control was not significantly different (good/very good pain control in $57 \%$ vs. $63 \%, \mathrm{p}=0.58$ ). Other self-reported functional outcomes did not significantly change (Table 2.3). 
Table 2.3 Pre-Intervention and Post-Intervention Comparison of Pain Control

\begin{tabular}{|c|c|c|c|}
\hline Primary and Secondary Outcomes & $\begin{array}{c}\text { Pre- } \\
\text { intervention } \\
\mathbf{N}=\mathbf{4 2}\end{array}$ & $\begin{array}{c}\text { Post- } \\
\text { intervention } \\
\mathbf{N}=\mathbf{5 1}\end{array}$ & $\begin{array}{c}\text { p- } \\
\text { value }\end{array}$ \\
\hline \multicolumn{4}{|l|}{ Average pain in first 7 post-operative days, (n) mean (SD) } \\
\hline All groups $(0-10)$ & $2.8(2.1)$ & $2.6(2.5)$ & 0.33 \\
\hline EUA $+/$ - seton placement $(n=11)$ & $3.3(3.1)$ & $2.4(2.9)$ & 0.31 \\
\hline Fistulotomy $(\mathrm{n}=37)$ & $2.0(1.6)$ & $1.9(1.8)$ & 0.44 \\
\hline Hemorrhoidectomy $(\mathrm{n}=13$ ) & $5.0(1.6)$ & $3.1(2.4)$ & 0.07 \\
\hline Pilonidal sinus excision or Abscess drainage $(n=7)$ & $2.0(2.6)$ & $4.5(2.5)$ & 0.13 \\
\hline Dilation, Advancement flap, Anal or rectal excision $(n=25)$ & $2.9(1.6)$ & $3.1(3.2)$ & 0.44 \\
\hline \multicolumn{4}{|l|}{ Quality of Pain Control (good/very good) } \\
\hline All groups n (\%) & $24(57)$ & $32(63)$ & 0.58 \\
\hline EUA +/- seton placement $\mathrm{n}(\%)$ & $2 / 6(33)$ & $3 / 5(60)$ & 0.57 \\
\hline Fistulotomy n (\%) & 10/15 (67) & $15 / 22(68)$ & 1.0 \\
\hline Hemorrhoidectomy n (\%) & $2 / 5(40)$ & $2 / 8(25)$ & 1.0 \\
\hline Pilonidal sinus/Abscess drainage $\mathrm{n}(\%)$ & $2 / 3(67)$ & $3 / 4(75)$ & 1.0 \\
\hline Dilation, Advancement flap, Anal/Rectal lesion excision $\mathrm{n}(\%)$ & $8 / 13(62)$ & $9 / 12(75)$ & 0.67 \\
\hline \multicolumn{4}{|l|}{ Patient function interference (all groups), mean (SD) } \\
\hline General Activity $(0-10)$ & $5.6(3.1)$ & $5.3(3.1)$ & 0.62 \\
\hline Walking ability $(0-10)$ & $4.8(3.1)$ & $4.3(3.2)$ & 0.52 \\
\hline Work $(0-10)$ & $5.0(3.2)$ & $5.0(3.4)$ & 0.93 \\
\hline Sleep $(0-10)$ & $4.5(3.1)$ & $3.5(2.9)$ & 0.14 \\
\hline Enjoyment $(0-10)$ & $5.3(3.2)$ & $4.5(3.2)$ & 0.19 \\
\hline
\end{tabular}

All outcomes are in means; (0-10) - eleven point numeric rating scale from modified Brief Pain Inventory: $0=$ no pain, $1-3=$ mild pain, $4-6=$ moderate pain, $7-10=$ severe pain; $0=$ no interference with function, $10=$ complete interference with function; Quality of pain control rated from very poor to very good (five point scale). ${ }^{27}$

\subsubsection{Medication Related Outcomes}

The median OME for prescriptions filled was significantly less after the intervention (112.5 [IQR 65-175] pre-intervention vs. 50 [IQR 50-50] post-intervention, $\mathrm{p}<0.001$ ) (Table 2.4). Only 23/51 (45\%) of post-intervention patients filled their opioid prescription, with no significant difference in prescription renewals (15.2\% pre-intervention vs. $4.3 \%$ post-intervention, $\mathrm{p}=0.20$ ). 
In addition, the median amount of opioids taken in the post-intervention group was reported at 12.5 OME (2.5 pills). Appropriate excess medication disposal did not significantly differ (2/32 [6.3\%] to 4/23 [17.4\%], $\mathrm{p}=0.10$ ), after the intervention was initiated (Table 2.4).

Table 2.4 Pre-Intervention and Post-Intervention Comparison of Medications

\begin{tabular}{|c|c|c|c|}
\hline Medication Comparison & $\begin{array}{c}\text { Pre- } \\
\text { intervention } \\
\mathrm{N}=\mathbf{4 2}\end{array}$ & $\begin{array}{c}\text { Post- } \\
\text { intervention } \\
\mathrm{N}=\mathbf{5 1}\end{array}$ & p-value \\
\hline \multicolumn{4}{|l|}{ Narcotic prescription given: } \\
\hline OME, median $\left(25^{\text {th }}, 75^{\text {th }}\right)$ & $112.5(50-150)$ & $50(50-50)$ & $<0.001$ \\
\hline No. of pills, median $\left(25^{\text {th }}, 75^{\text {th }}\right)$ & $30(10-30)$ & $10(10-10)$ & $<0.001$ \\
\hline \multicolumn{4}{|l|}{ Narcotic prescription used: } \\
\hline OME, median $\left(25^{\text {th }}, 75^{\text {th }}\right)$ & $62.5(37.5-135)$ & $12.5(0-46.9)$ & $<0.001$ \\
\hline No. of pills, median $\left(25^{\text {th }}, 75^{\text {th }}\right)$ & $15(10-30)$ & $2.5(0-6.9)$ & $<0.001$ \\
\hline Surgeons prescribing > median 50 OME $(\%)$ & $10 / 13(77)$ & $0 / 10(0)$ & $<0.001$ \\
\hline Narcotic prescription filled n $(\%)$ & $33 / 42(79)$ & $23 / 51(45)$ & $<0.001$ \\
\hline NSAID use, $\mathrm{n}(\%)$ & $15 / 42(36)$ & $28 / 51(55)$ & 0.07 \\
\hline Acetaminophen use, n (\%) & $14 / 42(33)$ & $31 / 51(61)$ & 0.01 \\
\hline Simultaneous NSAID + Acetaminophen use, n (\%) & $5 / 42(12)$ & $10 / 51(20)$ & 0.31 \\
\hline Metronidazole prescription given, $\mathrm{n}(\%)$ & $18 / 42(43)$ & $36 / 51(71)$ & 0.01 \\
\hline Laxative prescription given, $\mathrm{n}(\%)$ & $7 / 42(17)$ & $22 / 51(43)$ & 0.01 \\
\hline Prescription renewal, n (\%) & $5 / 33(15.2)$ & $1 / 23(4.3)$ & 0.20 \\
\hline Appropriate medication disposal, $\mathrm{n}(\%)$ & $2 / 32(6.3)$ & $4 / 23(17.4)$ & 0.10 \\
\hline
\end{tabular}

OME, Oral morphine equivalent; NSAID, Non-steroidal anti-inflammatory

Anti-inflammatory use did not increase significantly ( $36 \%$ to $55 \%, \mathrm{p}=0.07)$, while acetaminophen use increased significantly (33\% to 61\%), $\mathrm{p}=0.01$ ) (Table 2.4). Metronidazole prescriptions increased significantly $(43 \%$ to $71 \%, \mathrm{p}=0.01)$, as did laxative prescriptions $(17 \%$ to $43 \%, \mathrm{p}=0.01$ ) (Figure 2.2). In a subgroup of hemorrhoidectomy patients, there was $88 \%$ and $63 \%$ prescribing frequency of metronidazole and PEG, respectively, which was higher than in the overall group of anorectal procedures (Table 2.5). 
Figure 2.2

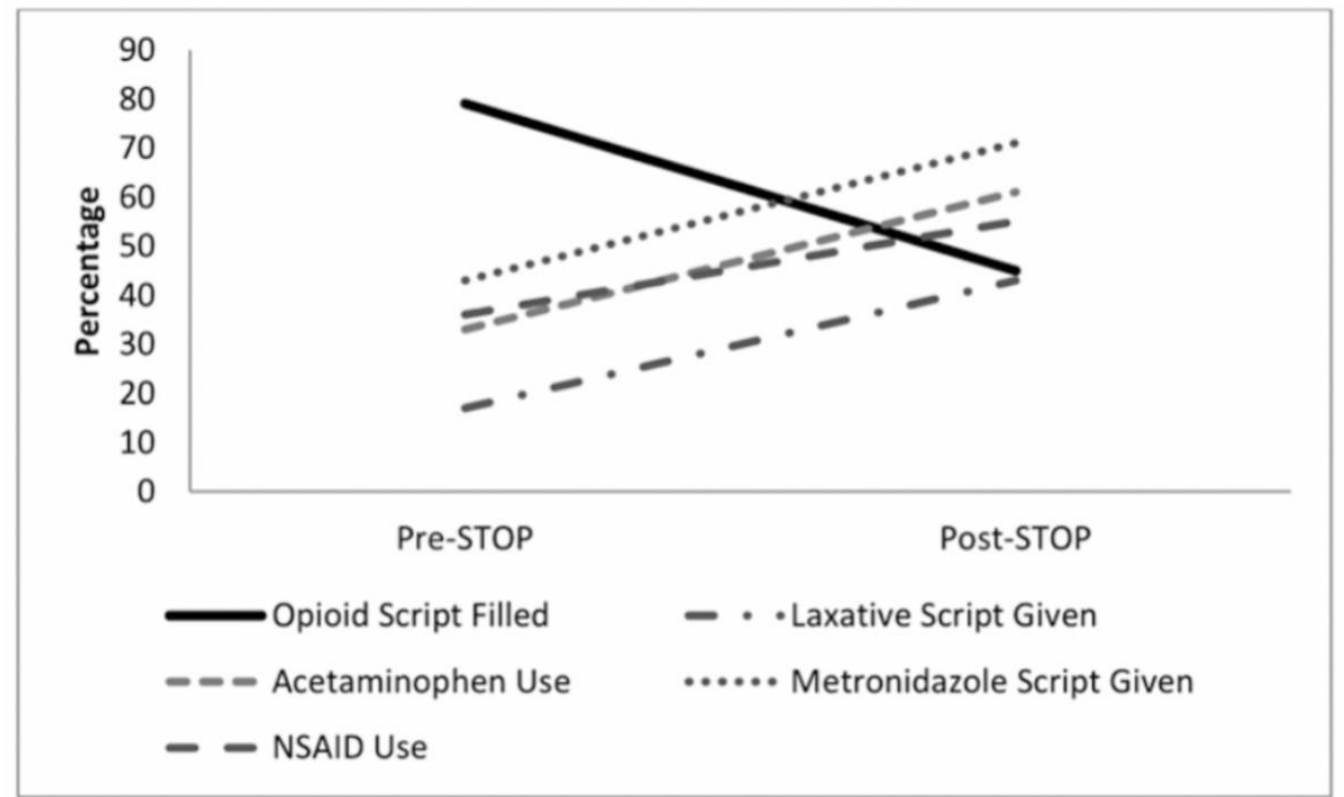

Figure 2.2 Utilization of Multi-Modal Analgesia Medications, Pre- and Post-Intervention

Table 2.5 Pre-Intervention and Post-Intervention Comparison: Hemorrhoidectomy Subgroup

\section{Hemorrhoidectomy}

\section{Pre-intervention}

$$
\mathbf{N}=\mathbf{5}
$$

$$
50(50-50)
$$

0.05

$30(10-30)$

$10(10-10)$

0.02

p-value

$\begin{array}{ccc}168.8(65.5-212.5) & 18.8(0-87.5) & \mathbf{0 . 0 3} \\ 30(14.4-42.5) & 3.8(0-6.3) & \mathbf{0 . 0 1} \\ 5 / 8(63) & 6 / 8(75) & 1.0 \\ 3 / 5(60) & 7 / 8(88) & 0.51 \\ 1 / 5(20) & 5 / 8(63) & 0.27 \\ 1 / 5(20) & 1 / 6(17) & 1.0\end{array}$

OME, Oral morphine equivalents; NSAID, Non-steroidal anti-inflammatory

\subsubsection{Surgeon and patient adherence}


The number of surgeons prescribing greater than a median of 50 OME was eliminated $(9 / 10$ [90\%] surgeons pre-intervention vs. $0 / 10$ surgeons post-intervention, $\mathrm{p}<0.001$ ). The most common patient-reported reasons for filling the opioid prescription were: the patient filled it just in case it was needed 11/23 (48\%), the patient was told to fill the prescription 5/23 (22\%), and it was needed for additional pain control 7/23 (30\%). As such, only 7/51 (14\%) of patients in the post-intervention group reported that they needed an opioid prescription for pain control (Tables 2.4 and 2.6).

Table 2.6 Post-intervention Education and Adherence

\begin{tabular}{lc} 
Patient adherence & $\begin{array}{c}\text { Post-intervention } \\
\mathbf{N}=\mathbf{5 1}\end{array}$ \\
\hline Patients received instruction sheet, $\mathrm{n}(\%)$ & $47(92)$ \\
\hline Patients found the instruction sheet helpful, $\mathrm{n}(\%)$ & $43(86)$ \\
\hline Reason for filling prescription, $\mathrm{n}=23$ & \\
\hline Patient was told to fill it, $\mathrm{n}(\%)$ & $5(22)$ \\
\hline Just in case the patient needed it, $\mathrm{n}(\%)$ & $11(48)$ \\
\hline For additional pain control, $\mathrm{n}(\%)$ & $7(30)$ \\
\hline
\end{tabular}

\subsubsection{Complications}

There were no documented incidents of upper gastrointestinal bleeding or renal failure in either the pre- or post-intervention groups.

\subsection{DISCUSSION:}

After implementation of the STOP Narcotics initiative in outpatient anorectal surgery, patientreported average pain scores in the first week after surgery were non-inferior to the preintervention care. Additionally, patient-rated quality of pain control was no different for all procedures. The STOP Narcotics intervention resulted in adequate analgesia for post-operative pain and significantly decreased OME prescribed and taken by patients, without an increase in prescription refills. 
Standardized patient care bundles and care pathways can be successfully used to institute multiple evidence-based interventions in a consistent way. Our intervention consisted of a multipronged approach, and similar to previous literature our opioid-reducing strategies consisted of patient and provider education, opioid-reduced prescriptions and multimodal non-opioid analgesia strategies. ${ }^{28-35}$ Verbal and written perioperative education, including clarifying patient expectations and providing non-opioid utilization instructions may reduce opioid use, consistent with our results. ${ }^{34}$

An optional limited prescription of an opioid with an expiry date of 7 days was provided in our initiative, as a back-up for those patients requiring additional pain control. Only $45 \%$ of patients filled this prescription and only $14 \%$ actually filled it for additional pain control, suggesting that this opioid sparing strategy is effective. Providing clear instructions and expectations to patients was important. Two recent Cochrane Reviews suggested ibuprofen and acetaminophen may in fact be more effective than opioids for post-operative analgesia. ${ }^{31,32}$ While compliance with the analgesia protocol in our study was not $100 \%$, these medications likely contributed significantly to opioid reduction. In addition, pre-emptive intra-operative analgesia with ketorolac and dexamethasone has been shown to decrease opioid requirements after surgery, with no increase in post-operative wound infection or other complications, which is consistent with the lack of major complications including gastrointestinal bleeding, renal failure, or thrombotic events in our study group. ${ }^{36-38}$ Using a patient care pathway has resulted in most patients not requiring any opioid prescription after anorectal surgery, in keeping with our previous work in other outpatient general surgical procedures. ${ }^{24}$

Oral metronidazole prescriptions were given as part of our anorectal surgery electronic order set, with $71 \%$ of patients receiving the prescription in the post-intervention group. A recent metaanalysis on metronidazole after hemorrhoidectomy demonstrated significantly lower reported pain on day 1 and 4 with significantly shorter time to return to normal activities, however, no difference was observed on most post-operative days. ${ }^{19,20}$ Despite this, we have elected to use it after anorectal surgery as part of our protocol, especially after hemorrhoidectomy, given the minimal side-effect profile, 
In the context of the current opioid crisis in North America, surgeons must recognize their role and act responsibly. A significant surgeon concern when prescribing less opioids may be the potential for an increased volume of patient calls for prescription refills, as well as decreased patient satisfaction. Our request for renewals was only $4.3 \%$ in the post-intervention group and less than previously reported by Sekhri, which is likely due to our study population of patients without significant chronic pain or opioid use, who were undergoing only outpatient surgeries. ${ }^{39}$ Interestingly, it has been shown that refill requests for pain medication do not correlate with smaller prescription sizes and that it is very uncommon for patients to use all of tablets prescribed for that episode of pain. ${ }^{10,39}$

Hemorrhoidectomy is often regarded as a painful surgery, ranking $23^{\text {rd }}$ of 529 procedures in patient-reported pain for well-defined surgical procedures. ${ }^{40}$ In our study, while there was no significant difference in average patient-reported pain after initiation of the STOP Narcotics intervention, only $2 / 8(25 \%)$ patients rated the quality of their pain control as "good or very good" in the post-intervention group (Table 2.3). This was not significantly different than the pre-intervention group. Despite the majority of patients filling their narcotic prescription in the hemorrhoidectomy group after the intervention (75\%), the median amount of opioid used was still very low (18.8 OME or 3.8 pills). This is significantly improved from the pre-intervention group (median 168.8 OME or 30 pills, $\mathrm{p}=0.03$ ). Of note, our subset of patients undergoing hemorrhoidectomy was small, and our study was not powered to specifically assess the impact in the patients undergoing hemorrhoidectomy. This is a subgroup which likely warrants further research on optimization of pain control.

An opioid should be only one component of pain management, and our approach is similar to the PROSPECT collaboration's conclusion that a combination of oral analgesics (NSAIDs, acetaminophen and opioids) and local anesthetic infiltration is recommended after anorectal surgery. ${ }^{22}$ While not part of the data captured in our study, all 10 surgeons reported routine use of local anesthetic when surveyed. Opioids cause constipation, which may significantly contribute to pain after anorectal surgery, and thus we have included a focus on avoiding constipation as part of our multimodality approach. 
Since study completion, our surgeon compliance with the STOP Narcotics care pathway has continued to be high. The initiative has been expanded to other procedures and other hospitals with positive feedback from multiple stakeholders. This is the first study in anorectal surgery showing that an opioid-sparing multi-modal pain management bundle is not inferior to traditional opioid prescribing practices. It is not, however, without limitations. There is inherent bias with the observational design and a randomized control trial was not performed due to a unified belief within the division that practice change regarding opioid prescribing was a priority. Our exclusion criteria are based on potential morbidities of the chosen analgesia components and have limited external validity. STOP Narcotics was implemented on a pre-specified date and there were likely some early changes in surgeon prescribing habits and patient education during the months before the initiative was officially implemented. Furthermore, there is variability between different anorectal procedures with regards to expected post-operative pain levels, and our study was powered to assess anorectal surgery as a single group. A future study specifically assessing patients undergoing hemorrhoidectomy may be useful to validate multi-modal pain management strategies in this subgroup. Additionally, local anesthetic and intra-operative medication data was not compared between the pre- and post-intervention groups, however, our purpose was not to evaluate their effectiveness, but to encourage their standard use. Finally, there was not complete compliance with the pain control initiatives by patients, nurses and surgeons.

\subsection{CONCLUSION:}

For outpatient anorectal surgeries requiring an anesthetic, a standardized care pain bundle (STOP Narcotics) significantly decreased opioid prescribing and often eliminated patient utilization of an opioid for pain control, without an increase in patient-reported post-operative pain. This was achieved through patient education, multi-modal opioid-sparing analgesia strategies, and focused systematic changes by nurses, anesthetists, and surgeons. While surgeons must consider the individual patent's needs for pain control after surgery, our study shows the value and impact of a standardized bundle to guide the care team and patient, while reducing opioid exposure after anorectal surgery. 


\subsection{REFERENCES}

1. Murphy SL, Xu J, Kenneth D, et al. National Vital Statistics Reports. https://www.cdc.gov/nchs/data/nvsr/nvsr66/nvsr66_06.pdf. Accessed January 14, 2019

2. Government of Canada: Apparent Opioid-Related Deaths in Canada. https://www.canada.ca/en/health-canada/services/substance-use/problematic-prescriptiondrug-use/opioids/apparent-opioid-related-deaths.html.Accessed December 10, 2018

3. Hill MV, McMahon ML, Stucke RS, Barth RJ. (2017) Wide Variation and Excessive Dosage of Opioid Prescriptions for Common General Surgical Procedures. Ann Surg. 265(4):709-714.

4. Thiels CA, Anderson SS, Ubl DS, et al. (2017) Wide Variation and Overprescription of Opioids After Elective Surgery. Ann Surg. 266(4):564-573.

5. Swarup A, Mathis KA, Hill MV, Ivatury SJ. (2018) Patterns of opioid use and prescribing for outpatient anorectal operations. J Surg Res.229:283-287.

6. Sun EC, Darnall BD, Baker LC, Mackey S. (2016) Incidence of and Risk Factors for Chronic Opioid Use Among Opioid-Naive Patients in the Postoperative Period. JAMA Intern Med.176(9):1286.

7. Brat G, Agniel D, Beam A, Yorkgitis B. (2018) Postsurgical prescriptions for opioid naive patients and association with overdose and misuse: retrospective cohort study. BMJ,360:j5790

8. Lev R, Lee O, Petro S, et al. (2016) Who is prescribing controlled medications to patients who die of prescription drug abuse? Am J Emerg Med. 34(1):30-35.

9. Place R, Hyman N, Simmang C, et al. (2003) Practice Parameters for Ambulatory Anorectal Surgery. Dis Colon Rectum. 46(5):573-576.

10. Fujii MH, Hodges AC, Russell RL, et al. (2018) Post-Discharge Opioid Prescribing and Use after Common Surgical Procedure. J Am Coll Surg. 226(6):1004-1012.

11. Overton HN, Hanna MN, Bruhn WE, et al. (2018) Opioid-Prescribing Guidelines for Common Surgical Procedures: An Expert Panel Consensus. J Am Coll Surg. 411-418.

12. Tan WH, Yu J, Feaman S, et al. Opioid Medication Use in the Surgical Patient: An Assessment of Prescribing Patterns and Use. (2018) J Am Coll Surg. 227(2):203-211.

13. Poylin V, Quinn J, Messer K, et al. (2014) Gabapentin significantly decreases posthemorrhoidectomy pain: a prospective study. Int J Colorectal Dis. 29(12):1565-1569.

14. Hancke E, Lampinski M, Suchan K, Völke K. (2013) Pain management after hemorrhoidectomy. Patient-controlled analgesia vs conventional pain therapy. Chirurg.84(7):587-593.

15. Rahimi M, Kazemeini AR, Pourtabatabaei N, Honarmand AR. (2012) Comparison of topical anesthetic cream (EMLA) and diclofenac suppository for pain relief after hemorrhoidectomy: a randomized clinical trial. Surg Today. 42(12):1201-1205. 
16. Tegon G, Pulzato L, Passarella L, et al. (2009) Randomized placebo-controlled trial on local applications of opioids after hemorrhoidectomy. Tech Coloproctol. 13(3):219-224.

17. Mohamed S, Kristensen B, Lingaard L, Bisgaard T. (2016) Acceptable effect of multimodal analgesic treatment after a Bascom cleft lift operation. Dan Med J. 1(62):A495.

18. Shim SM, Park JH, Hyun DM, et al. (2018) The effects of adjuvant intrathecal fentanyl on postoperative pain and rebound pain for anorectal surgery under saddle anesthesia. Korean J Anesthesiol. 71(3):213-219.

19. Lyons NJR, Cornille JB, Pathak S, et al. (2017) Systematic review and meta-analysis of the role of metronidazole in post-haemorrhoidectomy pain relief. Color Dis. 19(9):803811.

20. Wanis KN, Emmerton-Coughlin HM, Coughlin S, Vinden C. (2017) Systemic Metronidazole May Not Reduce Posthemorrhoidectomy Pain. Dis Colon Rectum. 60(4):446-455.

21. Balfour L, Stojkovic SG, Botterill ID, et al. (2002) A Randomized, Double-Blind Trial of the Effect of Metronidazole on Pain After Closed Hemorrhoidectomy. Dis Colon Rectum. 45(9):1186-1190.

22. Sammour T, Barazanchi AWH, Hill AG, (Collaborators) (2017) on behalf of the PROSPECT group. Evidence-Based Management of Pain After Excisional Haemorrhoidectomy Surgery: A PROSPECT Review Update. World J Surg. 41(2):603614.

23. Joint Statement of Action to Address the Opioid Crisis - Health Canada https://www.canada.ca/en/health-canada/services/substance-abuse/opioidconference/joint-statement-action-address-opioid-crisis.html.Accessed November 30, 2018.

24. Hartford LB, Van Koughnett JAM, Murphy PB, et al. (2019) Standardization of Outpatient Procedure (STOP) Narcotics: A Prospective Non-Inferiority Study to Reduce Opioid Use in Outpatient General Surgical Procedures. J Am Coll Surg. 228(1),81-88

25. The Strengthening the Reporting of Observation Studies in Epidemiology (STROBE). https://www.strobestatement.org/fileadmin/Strobe/uploads/checklists/STROBE_checklist_ v4_cohort.pdf.Accessed September 24, 2018

26. Keller S, Bann CM, Dodd SL, et al. (2004) Validity of the brief pain inventory for use in documenting the outcomes of patients with noncancer pain. Clin J Pain. 20(5):309-318.

27. Hawker GA, Mian S, Kendzerska T, French M. (2011) Measures of Adult Pain: Visual Analog Scale for Pain, Numeric Rating scale for pain, McGill pain questionnaire, Short form McGill pain questionnaire, Chronic pain grade scale, short form-36 bodily pain scale, measure of intermittent and constant osteoarthritis pain. Arthritis Care Res 63(11):240-52.

28. Farrar JT, Portenoy RK, Berlin JA, et al. (2000) Defining the clinically important difference in pain outcome measures. Pain. 88(3):287-294. 
29. Hill MV, Stucke RS, McMahon ML, Beeman JL, Barth RJ. (2018) An Educational Intervention Decreases Opioid Prescribing After General Surgical Operations. Ann Surg.267(3):468-472.

30. Wetzel M, Hockenberry J, Raval MV. (2018) Interventions for Postsurgical Opioid Prescribing A Systematic Review. JAMA Surg. 30322:1-7.

31. Derry S, Derry CJ, Moore RA. (2013) Single dose oral ibuprofen plus oxycodone for acute postoperative pain in adults. Cochrane Database Syst Rev.(6).

32. Derry CJ, Derry S, Moore RA. (2013) Single dose oral ibuprofen plus paracetamol (acetaminophen) for acute postoperative pain. Cochrane Database Syst Rev.(6).

33. Howard R, Waljee J, Brummett C, et al. (2018) Reduction in Opioid Prescribing Through Evidence-Based Prescribing Guidelines. JAMA Surg. 153(3):285.

34. Sugai DY, Deptula PL, Parsa AA, Don Parsa F. (2013) The importance of communication in the management of postoperative pain. Hawaii J Med Public Health. 72(6):180-184.

35. De Oliveira GS, Almeida MD, Benzon HT, McCarthy RJ. (2011) Perioperative Single Dose Systemic Dexamethasone for Postoperative PainA Meta-analysis of Randomized Controlled Trials. J Am Soc Anesthesiol. 115(3):575-588.

36. Van Backer JT, Jordan MR, Leahy DT, et al. (2018) Preemptive Analgesia Decreases Pain Following Anorectal Surgery. Dis Colon Rectum. 61(7),824-829

37. Place RJ, Coloma M, White PF, et al. (2000) Ketorolac improves recovery after outpatient anorectal surgery. Dis Colon Rectum. 43(6):804-808.

38. Coloma M, Duffy LL, White PF, et al. (2000) Dexamethasone Facilitates Discharge After Outpatient Anorectal Surgery. Anesth Analg. January 85-88.

39. Sekhri S, Arora NS, Cottrell H, et al. (2018) Probability of Opioid Prescription Refilling After Surgery: Does Initial Prescription Dose Matter? Ann Surg. 268(2):271-276.

40. Gerbershagen HJ, Aduckathil S, Van Wijck AJM, Peelen LM, Kalkman CJ, Meissner W. (2013) Pain Intensity on the First Day after Surgery. Anesthesiology. 118(4):934-944. 


\subsection{CHAPTER 3. THE STANDARDIZATION OF OUTPATIENT PROCEDURE (STOP) NARCOTICS: A PROSPECTIVE HEALTH SYSTEMS INTERVENTION TO REDUCE OPIOID USE IN AMBULATORY BREAST SURGERY}

Hartford LB, Van Koughnett JAM, Murphy PB, Allen LJ, Clarke CFM, Brackstone M, Gray DK, Maciver $A H$

\subsection{BACKGROUND}

North America has been faced with increasing dependence and misuse of prescription opioid medication. ${ }^{1,2}$ Women may become dependent on prescription opioid medication more quickly and are more at risk for overdose compared to men. ${ }^{3}$ Further, between 1999 and 2015, the rate of death from prescription opioid overdoses increased $471 \%$ among women, compared to a $218 \%$ increase among men. ${ }^{3}$

Surgeons may contribute to excess opioid prescriptions; in breast surgery alone $75-85 \%$ of opioid prescriptions remain unconsumed. ${ }^{4}$ Recent evidence also suggests that roughly $5-15 \%$ of opioid naïve patients continue to use opioids regardless of whether they have undergone major or minor procedures. ${ }^{5-9}$ Certainly, this is relevant to breast cancer patients, considering the recent trend to favourable prognosis, long-term survivorship and that cancer patients are particularly susceptible to opioid dependence. ${ }^{5-9}$

In breast surgery, there has been a shift from opioids as a primary analgesic to multimodal strategies. ${ }^{10-14}$ Opioid-sparing pre-emptive and post-operative pain management may include oral and intravenous non-opioids (such as acetaminophen, non-steroidal anti-inflammatories [NSAIDs], dexamethasone, gabapentin and ketamine), and local and regional nerve blocks (such

as paravertebral and pectoral). ${ }^{10-13,15-19}$ There is limited guidance in the context of breast surgery on the appropriate quantity of opioid medication to prescribe, duration of use and tapering strategies. $^{4,20,21}$ 
We identified opioid prescribing for acute pain as a priority area for improvement in our hospitals. ${ }^{22,23}$ Subsequently, the Standardization of Outpatient Procedure Narcotics (STOP Narcotics) was initiated as a health systems intervention. ${ }^{20}$ This tailored, opioid-sparing, multipronged strategy was developed with the objective of adequately controlling pain after outpatient surgery, while standardizing pain management and reducing opioid prescriptions.

This study examines the effectiveness of the STOP Narcotics initiative in ambulatory breast surgery procedures. Breast surgery was identified as a common surgical procedure which would benefit from a consistent, standardized protocol due to the variation of analgesic practices and likely over prescription of opioids at our institution. We hypothesized that compared to conventional practice, this opioid-sparing strategy would provide similar pain control while also reducing excess prescriptions and opioid use.

\subsection{METHODS}

\subsubsection{Study Design and Setting}

This prospective, non-inferiority pre- and post-intervention study was conducted at a singlecentre regional breast cancer care program at St. Joseph's Health Care, in London, Ontario, Canada. ${ }^{24}$ The multidisciplinary surgical team is composed of five general surgeons who regularly perform breast surgery, and is complemented by reconstructive plastic surgeons, breast imaging radiologists, nurse navigators, advanced practice nurses, spiritual care providers and social workers. ${ }^{24}$ All breast surgery performed at St. Joseph's Health Care is ambulatory surgery, with the exception of breast reconstruction. The Strengthening the Reporting of Observation studies in Epidemiology (STROBE) guidelines were followed. ${ }^{25}$ Ethics approval was obtained through the Health Sciences Research Ethics Board at Western University (HSREB\# 109651).

\subsubsection{Pre- and Post-Intervention Groups}

Inclusion criteria were patients aged 18 to 75 years, undergoing ambulatory breast surgery with general anesthetic [partial mastectomy (PM), simple mastectomy (SM), sentinel lymph node 
(SLN) biopsy, modified radical mastectomy (MRM), and axillary lymph node dissection (ALND)]. Reconstructive procedures were excluded, as these patients were admitted postoperatively. Patients with concurrent regular opioid use, co-existing chronic pain conditions (not including osteoarthritis or back pain), chronic kidney disease, or active peptic ulcer disease were excluded. Patients from the pre-intervention group (July 17, 2017 to October 18, 2017) were compared to the post-intervention group (October 23, 2017 to April 30, 2018). These two groups differed by exposure to the STOP Narcotics protocol.

\subsubsection{Intervention}

The intervention consisted of a tailored, multipronged approach focused on four strategies: patient education; health care provider education; opioid-sparing pre-emptive and post-operative analgesia strategies. This multidisciplinary plan was created with input from surgeons, anesthetists, nurses and patients and was a division-wide general surgery initiative. This opioidsparing strategy has been previously described in outpatient laparoscopic cholecystectomy and hernia procedures, and specific details for this protocol in breast surgery may be found in Appendix 5.3, 5.4. ${ }^{57}$

\subsubsection{Outcomes}

The primary outcome was average pain on an 11-point (0-10) numeric rating scale (NRS) during the first 7 post-operative days. ${ }^{27-29}$ This was captured at the first post-operative appointment

(typically 4 weeks at our institution) in a modified brief pain inventory survey. ${ }^{30,31}$ Secondary patient-related outcomes included overall quality of pain control [5-point scale (very poor to very good)] and functional outcomes [11-point NRS (0-10)]. ${ }^{31}$ Medication-related outcomes included the following: oral morphine equivalents (OME) prescribed and used; filling of opioid prescription (yes/no); NSAID use; acetaminophen use; prescription refills; excess medication disposal. Disposal was considered appropriate if the patient returned the medication to a pharmacy. 


\subsubsection{Statistical Analysis}

The sample size was calculated by hypothesizing our primary outcome would be non-inferior (post-intervention) to our control group (pre-intervention). Using an $\alpha$ of $0.025, \beta$ of 0.1 , and a non-inferiority meaningful clinical difference (MCD) of two on a numeric rating scale, 44 patients per group were required in each of the pre- and post-intervention groups. ${ }^{32}$ Statistical analysis was completed using SPSS Version 24 (SAS Institute, Cary NC, USA) ${ }^{33}$ Mann-Whitney U test and Student's t-test were used to assess for a difference between medians and means respectively. Non-normally distributed variables were expressed as medians with $25^{\text {th }}$ and $75^{\text {th }}$ quartiles, and normally distributed variables were assessed using means and standard deviations. Chi-squared was used to assess differences in categorical variables. Non-inferiority was tested using the two-sample, equal variance, t-test for mean difference. Only our primary outcome was tested for non-inferiority with a one-sided test, with a p-value of $<0.025$ considered significant. All other secondary variable were two-sided tests with a p-value of $<0.05$ considered significant.

\subsection{RESULTS}

\subsubsection{Study Population}

After exclusion, the study population consisted of 173 patients ( 85 pre-intervention, 88 postintervention), with a lower age in the post-intervention group (59 vs. 53 years, $p=0.004$ ), (Table 3.1). There were 7 patients excluded for additional reasons not specified in the exclusion criteria, as outlined in Figure 3.1. 
Figure 3.1

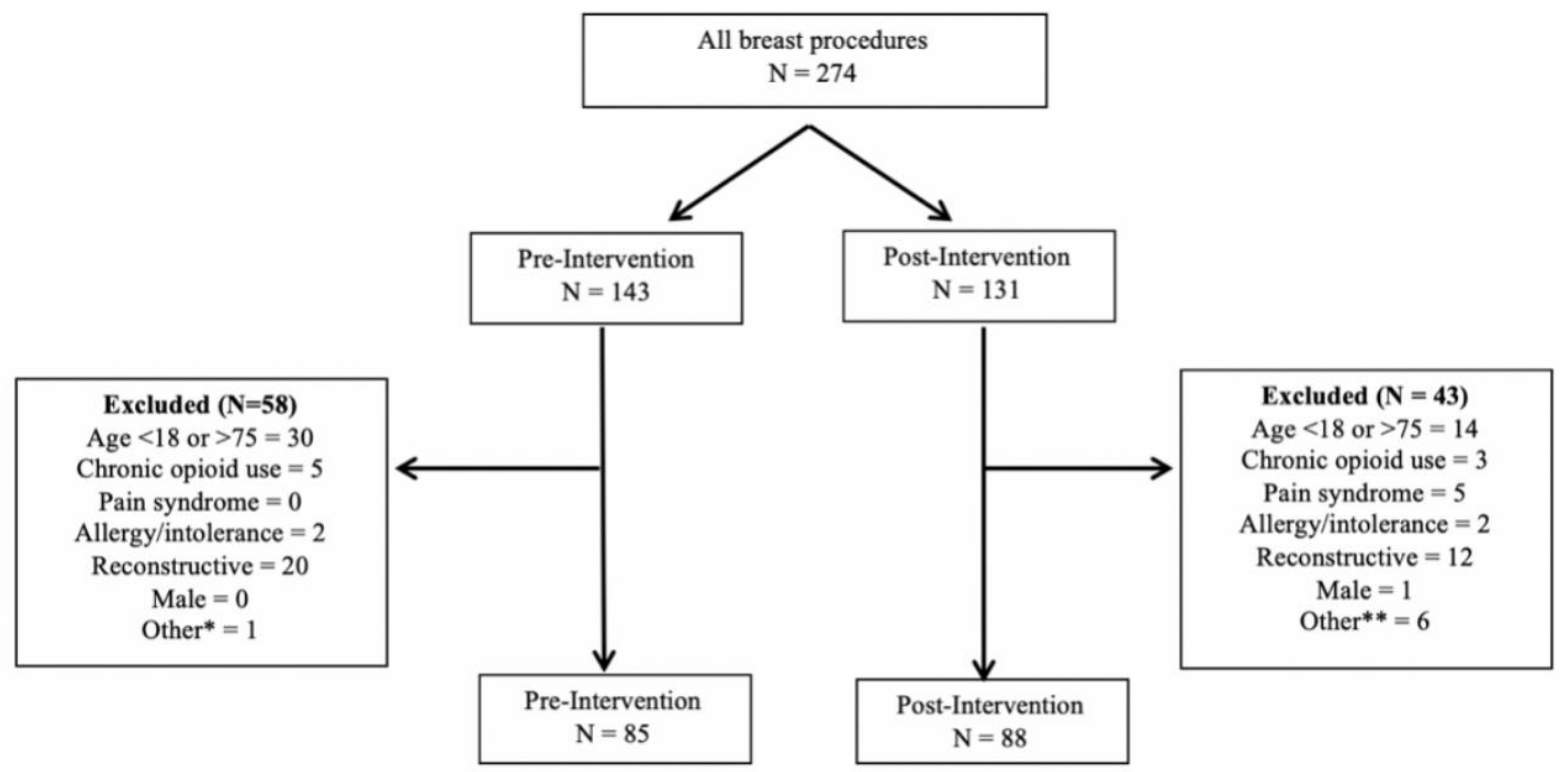

Figure 3.1 Flow chart of included patients. $* \mathrm{~N}=1$ (did not complete the brief pain inventory); $* * \mathrm{~N}=6$ ( 3 did not complete the brief pain inventory and 3 were inpatient procedures).

Table 3.1 Patient Characteristics

\begin{tabular}{lccc}
\hline Patients \& Procedures & $\begin{array}{c}\text { Pre-Intervention } \\
\mathbf{N = 8 5}\end{array}$ & $\begin{array}{c}\text { Post-Intervention } \\
\mathbf{N = 8 8}\end{array}$ & p-value \\
\hline Partial mastectomy & $28(33 \%)$ & $29(33 \%)$ & -- \\
Simple mastectomy & $8(9 \%)$ & $5(6 \%)$ & -- \\
\hline PM and SM & $\mathbf{3 6}(\mathbf{4 2 \%})$ & $\mathbf{3 4}(\mathbf{3 9 \%})$ & -- \\
\hline PM + SLN biopsy & $33(39 \%)$ & $33(38 \%)$ & -- \\
\hline SM + SLN biopsy & $12(14 \%)$ & $8(9 \%)$ & -- \\
\hline PM and SM + SLN biopsy & $\mathbf{4 5 ( 5 3 \% )}$ & $\mathbf{4 3 ( 4 9 \% )}$ & -- \\
\hline MRM & $1(1 \%)$ & $6(7 \%)$ & -- \\
PM + ALND & $1(1 \%)$ & $2(2 \%)$ & -- \\
\hline ALND & $2(2 \%)$ & $3(3 \%)$ & -- \\
MRM and ALND & $\mathbf{4 ( 5 \% )}$ & $\mathbf{1 1 ( 1 3 \% )}$ & -- \\
\hline Age (years), mean (SD) & $59(14)$ & $53(14)$ & 0.004 \\
\hline Female sex & $85(100 \%)$ & $88(100 \%)$ & 0.33 \\
\hline
\end{tabular}

PM, Partial Mastectomy; SM, Simple mastectomy; SLN, Sentinel lymph node biopsy; MRM, modified radical mastectomy; ALND, axillary lymph node dissection; SD, standard deviation 


\subsubsection{Primary Analgesia Outcome and Quality of Pain Control}

After implementation of the STOP Narcotics initiative, the primary outcome of average pain in the first 7 post-operative days was non-inferior to the control group (2.0/10 pre-intervention vs. 2.1/10 post-intervention, $\mathrm{p}=0.40$ ). This was comparable in the subgroup populations of $\mathrm{PM} / \mathrm{SM}$, SLN, or ALND patients when analyzed separately, (Table 3.2). Similarly, quality of pain control was not significantly different in any breast procedures (good/very good quality, $85 \%$ preintervention vs. $88 \%$ post-intervention, $\mathrm{p}=0.60$ ), or in the subgroup populations. Other functional self-reported outcomes did not significantly change, (Table 3.2).

Table 3.2 Pre- and Post-Intervention Comparison of Pain, Quality, and Function

\begin{tabular}{cccc}
\hline Primary and Secondary Outcomes & $\begin{array}{c}\text { Pre- } \\
\text { intervention } \\
\mathbf{N = \mathbf { 8 5 }}\end{array}$ & $\begin{array}{c}\text { Post- } \\
\text { intervention } \\
\mathbf{N}=\mathbf{8 8}\end{array}$ & p-value \\
\hline Average pain in first 7 post-operative days, mean (SD) & & \\
\hline All groups (0-10) & $2.0(1.8)$ & $2.1(1.6)$ & 0.40 \\
PM and SM (0-10) & $1.9(1.8)$ & $1.6(1.3)$ & 0.26 \\
PM and SM + SLN (0-10) & $2.1(1.8)$ & $2.4(1.7)$ & 0.20 \\
\hline MRM and ALND (0-10) & $2(2.2)$ & $2(1.9)$ & 0.50 \\
\hline Quality of Pain Control (good/very good) & & $77(88 \%)$ & 0.60 \\
\hline All groups & $72(85 \%)$ & $32(94 \%)$ & 0.09 \\
PM and SM & $29(81 \%)$ & $36(84 \%)$ & 0.70 \\
\hline PM and SM + SLN & $39(87 \%)$ & $9(82 \%)$ & 0.36 \\
\hline MRM and ALND & $4(100 \%)$ & & \\
\hline Patient function interference (all groups), mean (SD) & & $3.9(2.7)$ & 0.17 \\
\hline General Activity (0-10) & $3.3(3.0)$ & $1.2(2.1)$ & 0.28 \\
\hline Walking ability (0-10) & $1.5(2.6)$ & $3.7(3.3)$ & 0.32 \\
\hline Work (0-10) & $3.2(3.2)$ & $2.7(2.5)$ & 0.07 \\
\hline Sleep (0-10) & $3.4(2.9)$ & $2.6(2.6)$ & 0.47 \\
\hline Enjoyment (0-10) & $2.9(3.0)$ & &
\end{tabular}

All outcomes are in means; (0-10) - eleven-point numeric rating scale from modified brief pain inventory: $0=$ no pain, 1-3 = mild pain, 4-6 = moderate pain, 7-10 = severe pain; Quality of pain control - rated from very poor to very good (five-point scale); $0=$ no interference with function, $10=$ complete interference with function. ${ }^{27-29}$ 


\subsubsection{Opioid Prescriptions and Utilization}

Median OMEs and pills prescribed decreased from 100 OME (25 pills) pre-intervention vs. 50 OME (10 pills) post-intervention, $(\mathrm{p}<0.001)$. Patients consumed a median of 33.8 OME $(7.5$ pills) pre-intervention vs. 12.5 (2.5 pills) post-intervention, $(\mathrm{p}=0.002$ and $\mathrm{p}=0.007$, respectively). In the post-intervention group, only 39/88 (44\%) of patients filled their opioid prescription, compared to $64 / 75(85 \%)$ pre-intervention $(\mathrm{p}<0.001)$. Of the patients who filled their opioid prescription in the post-intervention group, 8/88 (9\%) patients reported filling it for pain not satisfactorily controlled with initial non-opioid alternative measures. The most common reason for filling the rescue opioid prescription was "just in case" (21 patients), followed by the patient being “instructed to fill it" (10 patients), (Table 3.3).

Table 3.3 Pre- and Post-Intervention Comparison of Medication

\begin{tabular}{|c|c|c|c|}
\hline Medication Comparison & $\begin{array}{l}\text { Pre-intervention } \\
\qquad \mathbf{N}=85\end{array}$ & $\begin{array}{l}\text { Post-intervention } \\
\qquad \mathbf{N}=\mathbf{8 8}\end{array}$ & p-value \\
\hline \multicolumn{4}{|l|}{ Narcotic prescription given: } \\
\hline OME, median $\left(25^{\text {th }}, 75^{\text {th }}\right)$ & $100(68-135)$ & $50(50-106)$ & $<0.001$ \\
\hline No. of pills, median $\left(25^{\text {th }}, 75^{\text {th }}\right)$ & $25(15-30)$ & $10(10-20)$ & $<0.001$ \\
\hline \multicolumn{4}{|l|}{ Narcotic prescription used: } \\
\hline OME, median $\left(25^{\text {th }}, 75^{\text {th }}\right)$ & $33.8(14-75)$ & $12.5(0-50)$ & 0.002 \\
\hline No. of pills, median $\left(25^{\text {th }}, 75^{\text {th }}\right)$ & $7.5(3.2-15)$ & $2.5(0-9.4)$ & 0.007 \\
\hline Surgeons prescribing $>$ median $50 \mathrm{OME}$ & $4 / 5(80 \%)$ & $1 / 5(20 \%)$ & 1.0 \\
\hline Narcotic prescription filled $*$ & $64 / 75(85 \%)$ & $39 / 88(44 \%)$ & $<0.001$ \\
\hline NSAID use & $30(35 \%)$ & $53(60 \%)$ & 0.001 \\
\hline Acetaminophen use & $54(64 \%)$ & $66(75 \%)$ & 0.10 \\
\hline Simultaneous NSAID + Acetaminophen use & $19(22 \%)$ & $50(57 \%)$ & $<0.001$ \\
\hline Prescription renewal* & $3 / 75(4 \%)$ & $2 / 88(2 \%)$ & 1.0 \\
\hline Appropriate medication disposal** & $6 / 50(12 \%)$ & $6 / 29(21 \%)$ & 0.04 \\
\hline
\end{tabular}

OME, Oral morphine equivalent; No., Number; NSAID, Non-steroidal anti-inflammatory; * different denominator as not all patients received an opioid prescription; ** different denominator as only included patients which did not use $100 \%$ of their opioid prescription 


\subsubsection{Analgesic Non-Opioid Adjuncts and Medication Disposal}

Patients' use of NSAIDs increased in the post-intervention group from $35 \%$ to $60 \%,(p=0.001)$, whereas acetaminophen use $(64 \%$ to $75 \%)$ was not statistically significant $(\mathrm{p}=0.10)$. Appropriate medication disposal increased from $12 \%$ to $21 \%(\mathrm{p}=0.04)$, (Table 3.3$)$.

\subsubsection{Subgroup Population Analysis}

In the subgroup population of SM/PM, SLN, and ALND, OMEs/pills prescribed decreased significantly in all groups, with the exception of ALND [135 OME (36 pills) pre-intervention vs. $50 \mathrm{OME}(10$ pills $)$ post-intervention, $(\mathrm{p}=0.08)]$. In procedures including axillary surgery, these patients did not fill their opioid prescription more frequently than breast surgery alone [SM/PM $=9 / 34(26 \%)$ vs. SLN = 23/43 (53\%) and ALND = 6/11 (55\%), $(\mathrm{p}=0.07)]$ or consume significantly more opioid pills [SM/PM 1.3 pills vs. SLN and ALND 2.5 pills, $(\mathrm{p}=0.63)$ ], (Table $3.4)$. 
Table 3.4 Medication Comparison in PM/SM, PM/SM + SLN, and ALND/MRM

\begin{tabular}{|c|c|c|c|}
\hline Medication Comparison & $\begin{array}{l}\text { Pre-intervention } \\
\qquad \mathbf{N}=\mathbf{8 5}\end{array}$ & $\begin{array}{c}\text { Post-intervention } \\
\quad \mathbf{N}=\mathbf{8 8}\end{array}$ & p-value \\
\hline PM and SM & $\mathbf{N}=36$ & $\mathbf{N}=34$ & \\
\hline \multicolumn{4}{|l|}{ Narcotic prescription given: } \\
\hline OME, median $\left(25^{\text {th }}, 75^{\text {th }}\right)$ & $75(56-135)$ & $50(50-98)$ & 0.016 \\
\hline No. of pills, median $\left(25^{\text {th }}, 75^{\text {th }}\right)$ & $19(15-30)$ & $10(10-16)$ & 0.008 \\
\hline \multicolumn{4}{|l|}{ Narcotic prescription used: } \\
\hline OME, median $\left(25^{\text {th }}, 75^{\text {th }}\right)$ & $30(14-75)$ & $6(0-50)$ & 0.091 \\
\hline No. of pills, median $\left(25^{\text {th }}, 75^{\text {th }}\right)$ & $7.5(3.8-15)$ & $1.3(0-8.1)$ & 0.062 \\
\hline Narcotic prescription filled & $27(84 \%)$ & $9(28 \%)$ & $<0.001$ \\
\hline PM and SM + SLN & $\mathrm{N}=45$ & $\mathrm{~N}=43$ & \\
\hline \multicolumn{4}{|l|}{ Narcotic prescription given: } \\
\hline OME, median $\left(25^{\text {th }}, 75^{\text {th }}\right)$ & $100(75-135)$ & $50(50-113)$ & 0.033 \\
\hline No. of pills, median $\left(25^{\text {th }}, 75^{\text {th }}\right)$ & $25(20-30)$ & $10(10-30)$ & 0.011 \\
\hline \multicolumn{4}{|l|}{ Narcotic prescription used: } \\
\hline OME, median $\left(25^{\text {th }}, 75^{\text {th }}\right)$ & $38(14-75)$ & $13(0-50)$ & 0.066 \\
\hline No. of pills, median $\left(25^{\text {th }}, 75^{\text {th }}\right)$ & $7.5(3.4-15)$ & $2.5(0-10)$ & $\mathbf{0 . 0 3 0}$ \\
\hline Narcotic prescription filled & $33(85 \%)$ & $23(54 \%)$ & 0.002 \\
\hline ALND and MRM & $\mathbf{N}=4$ & $\mathbf{N}=\mathbf{1 1}$ & \\
\hline \multicolumn{4}{|l|}{ Narcotic prescription given: } \\
\hline OME, median $\left(25^{\text {th }}, 75^{\text {th }}\right)$ & $135(113-208)$ & $50(50-50)$ & 0.08 \\
\hline No. of pills, median $\left(25^{\text {th }}, 75^{\text {th }}\right)$ & $36(30-55.5)$ & $10(10-10)$ & $\mathbf{0 . 0 3}$ \\
\hline \multicolumn{4}{|l|}{ Narcotic prescription used: } \\
\hline OME, median $\left(25^{\text {th }}, 75^{\text {th }}\right)$ & $20(0-94)$ & $13(0-38)$ & 0.79 \\
\hline No. of pills, median $\left(25^{\text {th }}, 75^{\text {th }}\right)$ & $5.25(0-25)$ & $2.5(0-7.5)$ & 0.65 \\
\hline Narcotic prescription filled & $4(100 \%)$ & $6(55 \%)$ & 0.10 \\
\hline
\end{tabular}

PM, Partial mastectomy; SM, Simple mastectomy; SLN, Sentinel lymph node biopsy; MRM, Modified radical mastectomy; ALND, Axillary lymph node dissection; SD, Standard deviation; OME, Oral morphine equivalents; NSAID, Non-steroidal anti-inflammatory

\subsubsection{Patient and Surgeon Compliance}

Patients reported receiving the education sheet $92 \%$ of the time, with $88 \%$ confirming it to be helpful. Surgeons prescribing greater than 50 OMEs for breast procedures decreased from 4/5 $(80 \%)$ to $1 / 5(20 \%)$. 


\subsubsection{Complications}

There were no documented incidents of upper gastrointestinal bleeding or renal failure in either cohort. There were no reports of skin flap necrosis or other known complications from topical ice therapy.

\subsection{DISCUSSION}

The major findings from this study are three-fold: first, after implementation of a multi-pronged, opioid-sparing initiative (STOP Narcotics), control of acute post-operative pain in the first 7 days was not inferior to pre-implementation practice. Second, patient education, health care provider education, opioid-reduced prescriptions, and multimodal non-opioid analgesic strategies were reaffirmed to be effective in significantly reducing both prescribed and consumed opioids. ${ }^{11,13,17,34-38}$ Finally, these strategies may be integrated effectively into a perioperative pain control bundle, and implemented as a health systems intervention for outpatient breast procedures. ${ }^{36,37,39-41}$

Our findings have important implications. Common reasons from providers for large opioid prescriptions are the minimization of refill requests and ensuring adequate analgesia. While our inclusion criteria included elective, low risk patients, refill requests did not correlate with prescription strength or amount, similar to other studies. ${ }^{26,38,42}$ Our study also affirms that actual amount of opioid consumed is often a fraction of that which is prescribed. ${ }^{43}$ Indeed, only $44 \%$ of patients filled their rescue opioid prescription, consuming a median of 7.5 opioid pills and 2.5 opioid pills in our pre- and post-intervention groups respectively. This is similar to findings of Hill et al, who reported patients only used $14.7 \%$ of their opioid prescription following partial mastectomy and $25.7 \%$ after SLN biopsy. ${ }^{4,44-46}$ Considering the average pain score was 2.0 and 2.1 in our pre- and post-intervention group (threshold for moderate pain $\geq 4 / 10$ on the 11-point NRS), the minimal amount of opioid consumed is providing adequate analgesia. ${ }^{32,47,48}$ Surgeons may not be providing realistic expectations of postoperative discomfort, and underestimating the effectiveness of opioid-sparing, multimodal analgesic medications, often reported to be equally 
effective in the literature. ${ }^{49,50}$ In fact, rescue opioid therapy was only needed in 8/88 (9\%) of our patients, for discomfort not adequately controlled with acetaminophen, NSAIDs, and conservative measures.

A rescue opioid prescription with an expiry date of 7 days was a novel component of the STOP Narcotics initiative, preventing excess and unused medication available for unintended purposes in the future. ${ }^{26}$ This is in keeping with recent breast surgery guidelines supporting non-opioid multimodal analgesia with rescue opioids if needed. ${ }^{10,51}$ With only $9 \%$ of patients filling the rescue prescription for "additional pain control", the most common reason patients filled the prescription was "just in case" (24\%) or the patient "was told to fill it" (11\%). This may be related to inadequate access to a 24-hour pharmacy or advice of pharmacists. There was no data collected on patient geography to confirm this. Despite specific written instructions, appropriate medication disposal only increased to $21 \%$, consistent with other published interventions. ${ }^{26,52,53}$ Future studies are indicated to assess methods that will improve rates of appropriate narcotic disposal. ${ }^{26,52,53}$

Procedures involving the axilla, particularly ALND, are associated with greater discomfort and higher rates of chronic pain. ${ }^{54}$ There was no significant difference in average patient-reported pain post SLN biopsy or ALND in our post-intervention group in the first 7 post-operative days. Despite more patients filling their prescription in the SLN and ALND subgroups, the amount of reported opioid consumed was still very low (12.5 OME). With more extensive dissection in a highly innervated area, and with a higher risk of chronic pain syndromes and mobility complications, future studies with larger populations and longer follow up may be indicated to further define additional analgesic adjuncts for these procedures.

In accordance with previous studies, patient and provider education are key opioid-reducing modalities. ${ }^{34,35}$ Complete elimination of pain is not a reasonable expectation, and a mild amount of discomfort is normal and should be expected by patients. ${ }^{20} \mathrm{Katz}$ et al. demonstrated how preoperative anxiety was an important variable contributing to the prediction of acute pain after surgery. ${ }^{55} \mathrm{We}$ believe a short conversation between the patient and the caregiver with the health care provider about expectations, the recovery process, and optimal use of non-opioid 
medication, reinforced with a standardized education sheet was key in this initiative. ${ }^{56}$ Providers need to be educated on the actual amount of opioid patients consume after breast surgery, nonopioid analgesic options, and how to well-inform their patients of analgesia options. ${ }^{35}$

This study has many strengths including being one of the first published multi-pronged, opioidsparing pain care bundles to be introduced in breast surgery, and is replicable for other health

systems. ${ }^{26,40}$ However, it is not without limitations. There was a difference in patient age between pre/post-implementation periods where patients in the latter were younger. We believe this was due to chance and it is unlikely to impact our outcome, post-operative pain. There is inherent bias with an observational pre-/post study design. With this design practice may have changed prior the official implementation of the intervention. However, if this occurred it would underestimate the change in prescribing, as retrospectively surgeons were prescribing even higher quantities of opioids. Another limitation is single-centre data with sequential enrollment of patients and potentially limited external validity in other centres and in patients who do not meet inclusion criteria. However, with lower rates of resident turnover and variability in nonacademic centres, we believe it may be equally effective in those settings. We have implemented STOP Narcotics in additional community hospitals and continue to collect data from these centres. Further work in analysis of findings from STOP Narcotics protocol in other communities will further inform the generalizability of this work. It is important to note that although local anesthetic field blocks were not part of the official STOP Narcotics protocol, they are used regularly by all five surgeons. Extended-release local anesthetic and additional non-opioid coanalgesics could be added to future studies and could also be incorporated into a similar protocol.

Considering there are over 20,000 outpatient breast surgeries in the province of Ontario per year, the STOP Narcotics initiative could potentially decrease opioids after surgery from 500,000 to 88,000 pills.

\subsection{CONCLUSION}

Adequate analgesia may be obtained for outpatient breast surgery, using a multipronged pain care pathway targeting patient and provider education, as well as multimodal, opioid-sparing 
intra- and post-operative analgesia strategies (STOP Narcotics initiative). Opioid use can be significantly reduced and often eliminated when used as rescue therapy. A culture shift is needed in order to implement non-opioid alternatives as the foundation of pain management for outpatient surgical breast procedures. This initiative may be replicable at a health systems level and provide a framework for future analgesia guidelines in ambulatory breast surgery.

\subsection{REFERENCES}

1. Dart RC, Surratt HL, Cicero TJ, Parrino MW, Severtson SG, Bucher-Bartelson B, Green JL. Trends in Opioid Analgesic Abuse and Mortality in the United States. $N$ Engl J Med. 2015;372(3):241-248. doi:10.1056/NEJMsa1406143

2. Government of Canada - Health Canada (2017) National report: Apparent opioid-related deaths in Canada Available: https://www.canada.ca/en/publichealth/services/publications/healthy-living/apparent-opioid-related-deaths-report-20162017-december.html. Accessed November 10, 2018.

3. U.S. Department of Health and Human Services. (2017) Final Report: Opioid Use, Misuse, and Overdose in Women. Available: https://www.womenshealth.gov/files/documents/final-report-opioid-508.pdf. Accessed November 10, 2018.

4. Hill M V., McMahon ML, Stucke RS, Barth RJ. Wide Variation and Excessive Dosage of Opioid Prescriptions for Common General Surgical Procedures. Ann Surg. 2017;265(4):709-714. doi:10.1097/SLA.0000000000001993

5. Marcusa DP, Mann RA, Cron DC, et al. Prescription Opioid Use among Opioid-Naive Women Undergoing Immediate Breast Reconstruction. Plast Reconstr Surg. 2017;140(6):1081-1090. doi:10.1097/PRS.0000000000003832

6. Brummett CM, Waljee JF, Goesling J, et al. New Persistent Opioid Use After Minor and Major Surgical Procedures in US Adults. JAMA Surg. 2017;152(6):e170504.

doi:10.1001/jamasurg.2017.0504

7. Sun EC, Darnall BD, Baker LC, Mackey S. Incidence of and Risk Factors for Chronic Opioid Use Among Opioid-Naive Patients in the Postoperative Period. JAMA Intern Med. 2016;176(9):1286. doi:10.1001/jamainternmed.2016.3298

8. Carmichael A-N, Morgan L, Del Fabbro E. Identifying and assessing the risk of opioid abuse in patients with cancer: an integrative review. Subst Abuse Rehabil. 2016;7:71-79. doi:10.2147/SAR.S85409 
9. Lee JS-J, Hu HM, Edelman AL, et al. New Persistent Opioid Use Among Patients With Cancer After Curative-Intent Surgery. J Clin Oncol. 2017;35(36):4042-4049. doi:10.1200/JCO.2017.74.1363

10. Afonso AM, Newman MI, Seeley N, et al. Multimodal Analgesia in Breast Surgical Procedures: Technical and Pharmacological Considerations for Liposomal Bupivacaine Use. Plast Reconstr surgery Glob open. 2017;5(9):e1480. doi:10.1097/GOX.0000000000001480

11. Gärtner R, Kroman N, Callesen T, Kehlet H. Multimodal prevention of pain, nausea and vomiting after breast cancer surgery. Minerva Anestesiol. 2010;76(10):805-813. http://www.ncbi.nlm.nih.gov/pubmed/20935616. Accessed November 11, 2018.

12. Kumar K, Kirksey MA, Duong S, Wu CL. A Review of Opioid-Sparing Modalities in Perioperative Pain Management. Anesth Analg. 2017;125(5):1749-1760.

doi:10.1213/ANE.0000000000002497

13. Elvir-Lazo OL, White PF. The role of multimodal analgesia in pain management after ambulatory surgery. Curr Opin Anaesthesiol. 2010;23(6):697-703.

doi:10.1097/ACO.0b013e32833fad0a

14. Offodile AC, Gu C, Boukovalas S, Coroneos CJ, Chatterjee A, RD Largo, Butler C. Enhanced recovery after surgery (ERAS) pathways in breast reconstruction: systematic review and meta-analysis of the literature. Breast Cancer Res Treat. October 2018:1-13. doi:10.1007/s10549-018-4991-8

15. Fassoulaki A, Triga A, Melemeni A, Sarantopoulos C. Multimodal Analgesia with Gabapentin and Local Anesthetics Prevents Acute and Chronic Pain After Breast Surgery for Cancer. Anesth Analg. 2005;101(5):1427-1432.

doi:10.1213/01.ANE.0000180200.11626.8E

16. Karmakar MK, Samy W, Li JW, Lee A, WC Chan, Chen PP, AMH Ho,. Thoracic Paravertebral Block and Its Effects on Chronic Pain and Health-Related Quality of Life After Modified Radical Mastectomy. Reg Anesth Pain Med. 2014;39(4):289-298. doi:10.1097/AAP.0000000000000113

17. Gómez-Hernández J, Orozco-Alatorre AL, Domínguez-Contreras M, et al. Preoperative dexamethasone reduces postoperative pain, nausea and vomiting following mastectomy for breast cancer. BMC Cancer. 2010;10(1):692. doi:10.1186/1471-2407-10-692

18. Correll, Darin J; Viscusi, Eugene R; Grunwald, Zvi; Moore JHJ. Epidural Analgesia Compared With Intravenous Morphine Patient-Controlled Analgesia: Postoperative Outcome Measures After Mastectomy With Immediate TRAM Flap Breast Reconstruction. Reg Anesth Pain Med. 2001;26(5):444-449. 
19. Chang YC, Liu CL, Liu TP, Yang PS, Chen MJ, Cheng SP. Effect of Perioperative Intravenous Lidocaine Infusion on Acute and Chronic Pain after Breast Surgery: A MetaAnalysis of Randomized Controlled Trials. Pain Pract. 2017;17(3):336-343. doi:10.1111/papr.12442

20. Scully RE, Schoenfeld AJ, Jiang W, et al. Defining Optimal Length of Opioid Pain Medication Prescription After Common Surgical Procedures. JAMA Surg. 2018;153(1):37. doi:10.1001/jamasurg.2017.3132

21. Lee JS, Howard RA, Klueh MP, et al. The Impact of Education and Prescribing Guidelines on Opioid Prescribing for Breast and Melanoma Procedures. Ann Surg Oncol. September 2018. doi:10.1245/s10434-018-6772-3

22. Centers for Disease Control and Prevention. (2018) CDC's Response to the Opioid Overdose Epidemic. Available: https://www.cdc.gov/opioids/strategy.html. Accessed November 11, 2018.

23. Health Canada. (2017) Joint Statement of Action to Address the Opioid Crisis: A Collective Response. Available: http://www.ccsa.ca/Resource Library/CCSA-JointStatement-of-Action-Opioid-Crisis-Annual-Report-2017-en.pdf. Accessed November 11, 2018.

24. Breast Care Program | St. Joseph's Health Care London. (2019) Available: https://www.sjhc.london.on.ca/breastcare. Accessed December 8, 2018.

25. The Strengthening the Reporting of Observation Studies in Epidemiology (STROBE). (2019) Available:

https://www.strobestatement.org/fileadmin/Strobe/uploads/checklists/STROBE_checklist_ v4_cohort.pdf accessed June 5th.

26. Hartford LB, Van Koughnett JAM, Murphy PB, et al. Standardization of Outpatient Procedure (STOP) Narcotics: A Prospective Non-Inferiority Study to Reduce Opioid Use in Outpatient General Surgical Procedures. J Am Coll Surg. October 2018. doi:10.1016/J.JAMCOLLSURG.2018.09.008

27. Krebs EE, Carey TS, Weinberger M. Accuracy of the Pain Numeric Rating Scale as a Screening Test in Primary Care. J Gen Intern Med. 2007;22(10):1453-1458. doi:10.1007/s11606-007-0321-2

28. Jensen, M.P.; Karoly P. Self-report scales and procedures for assessing pain in adults. PsycNET. https://psycnet.apa.org/record/1992-98469-004. Published 1992. Accessed May $1,2019$.

29. Jensen MP, Turner JA, Romano JM, Fisher LD. Comparative reliability and validity of chronic pain intensity measures. Pain. 1999;83(2):157-162. doi:10.1016/S03043959(99)00101-3 
30. Hawker GA, Mian S, Kendzerska T, French M. Measures of Adult Pain: Visual Analog Scale for Pain, Numeric Rating scale for pain, McGill pain questionnaire, Short form McGill pain questionnaire, Chronic pain grade scale, short form-36 bodily pain scale, measure of intermittent and constant osteoarthritis pain. Arthritis Care Res 2011;63(11):240-52 doi:10.1002/acr.20543

31. Keller S, Bann CM, Dodd SL, Schein J, Mendoza TR, Cleeland CS. Validity of the brief pain inventory for use in documenting the outcomes of patients with noncancer pain. Clin J Pain. 20(5):309-318. http://www.ncbi.nlm.nih.gov/pubmed/15322437. Accessed June 4, 2018.

32. Gerbershagen HJ, Rothaug J, Kalkman CJ, Meissner W. Determination of moderate-tosevere postoperative pain on the numeric rating scale: a cut-off point analysis applying four different methods. Br J Anaesth. 2011;107(4):619-626. doi:10.1093/bja/aer195

33. IBM Corp. IBM SPSS Statistics for Windows, Released 2016, Version 24.0. Armonk, NY

34. Sugai DY, Deptula PL, Parsa AA, Don Parsa F. The importance of communication in the management of postoperative pain. Hawaii J Med Public Health. 2013;72(6):180-184. http://www.ncbi.nlm.nih.gov/pubmed/23795326. Accessed November 4, 2018.

35. Hill M V., Stucke RS, McMahon ML, Beeman JL, Barth RJ. An Educational Intervention Decreases Opioid Prescribing After General Surgical Operations. Ann Surg. 2018;267(3):468-472. doi:10.1097/SLA.0000000000002198

36. Wetzel M, Hockenberry J, Raval M V. Interventions for Postsurgical Opioid Prescribing. JAMA Surg. 2018;153(10):948. doi:10.1001/jamasurg.2018.2730

37. Meisenberg BR, Grover J, Campbell C, Korpon D. Assessment of Opioid Prescribing Practices Before and After Implementation of a Health System Intervention to Reduce Opioid Overprescribing. JAMA Netw Open. 2018;1(5):e182908. doi:10.1001/jamanetworkopen.2018.2908

38. Howard R, Waljee J, Brummett C, Englesbe M, Lee J. Reduction in Opioid Prescribing Through Evidence-Based Prescribing Guidelines. JAMA Surg. 2018;153(3):285. doi:10.1001/jamasurg.2017.4436

39. Chiu C, Aleshi P, Esserman LJ, Inglis-Arkell C, Yap E, Whitlock EL, Harbell MW,. Improved analgesia and reduced post-operative nausea and vomiting after implementation of an enhanced recovery after surgery (ERAS) pathway for total mastectomy. BMC Anesthesiol. 2018;18(1):41. doi:10.1186/s12871-018-0505-9

40. Rojas KE, Manasseh DM, Flom PL, Agbroko S, Bilbro N, Andaz C, Borgen PI. A pilot study of a breast surgery Enhanced Recovery After Surgery (ERAS) protocol to eliminate narcotic prescription at discharge. Breast Cancer Res Treat. 2018;171(3):621-626. 
doi:10.1007/s10549-018-4859-y

41. Arsalani-Zadeh R, ELFadl D, Yassin N, MacFie J. Evidence-based review of enhancing postoperative recovery after breast surgery. Br J Surg. 2011;98(2):181-196.

doi:10.1002/bjs.7331

42. Sekhri S, Arora NS, Cottrell H, et al. Probability of Opioid Prescription Refilling After Surgery: Does Initial Prescription Dose Matter? Ann Surg. 2018;268(2):271-276.

doi:10.1097/SLA.0000000000002308

43. Waljee JF, Li ÂL, Brummett CM, Englesbe MJ. Iatrogenic Opioid Dependence in the United States Are Surgeons the Gatekeepers? 2017;265(4):728-730.

doi:10.1097/SLA.0000000000001904

44. Fujii MH, Hodges AC, Russell RL, et al. Post-Discharge Opioid Prescribing and Use after Common Surgical Procedure. J Am Coll Surg. 2018;226(6):1004-1012.

doi:10.1016/j.jamcollsurg.2018.01.058

45. Tan WH, Yu J, Feaman S, et al. Opioid Medication Use in the Surgical Patient: An Assessment of Prescribing Patterns and Use. J Am Coll Surg. 2018;227(2):203-211. doi:10.1016/J.JAMCOLLSURG.2018.04.032

46. Feinberg AE, Chesney TR, Srikandarajah S, Acuna SA, McLeod RS. Opioid Use after Discharge in Postoperative Patients: A Systematic Review. Ann Surg. 2018;267(6):10561062. doi:10.1097/SLA.0000000000002591

47. Andersen L, Gaarn-Larsen L, Kristensen BB, Husted H, Otte KS, Kehlet H. Subacute pain and function after fast-track hip and knee arthroplasty. Anaesthesia. 2009;64(5):508-513. doi:10.1111/j.1365-2044.2008.05831.x

48. Breivik H, Borchgrevink PC, Allen SM, et al. Assessment of pain. Br J Anaesth. 2008;101(1):17-24. doi:10.1093/bja/aen103

49. Derry CJ, Derry S, Moore RA. Single dose oral ibuprofen plus paracetamol (acetaminophen) for acute postoperative pain. Cochrane Database Syst Rev. 2013;(6). doi:10.1002/14651858.CD010210.pub2

50. Derry S, Derry CJ, Moore RA. Single dose oral ibuprofen plus oxycodone for acute postoperative pain in adults. Cochrane Database Syst Rev. 2013;(6). doi:10.1002/14651858.CD010289.pub2

51. Overton HN, Hanna MN, Bruhn WE, et al. Opioid-Prescribing Guidelines for Common Surgical Procedures: An Expert Panel Consensus. J Am Coll Surg. 2018;227(4):411-418. doi:10.1016/J.JAMCOLLSURG.2018.07.659

52. Reddy A, de la Cruz M, Rodriguez EM, et al. Patterns of Storage, Use, and Disposal of 
Opioids Among Cancer Outpatients. Oncologist. 2014;19(7):780-785.

doi:10.1634/theoncologist.2014-0071

53. Hasak JM, Bettlach CLR, Santosa KB, Stroud J, Mackinnon SE, Larson EL. Empowering Post-Surgical Patients to Improve Opioid Disposal : A Before and After Quality Improvement Study. J Am Coll Surg. 2018;226(3):235-240.e3.

doi:10.1016/j.jamcollsurg.2017.11.023

54. Gärtner R, Jensen M-B, Nielsen J, Ewertz M, Kroman N, Kehlet H. Prevalence of and Factors Associated With Persistent Pain Following Breast Cancer Surgery. JAMA. 2009;302(18):1985. doi:10.1001/jama.2009.1568

55. Katz J, Poleshuck EL, Andrus CH, Hogan LA, Jung BF, Kulick DI, Dworkin RH. Risk factors for acute pain and its persistence following breast cancer surgery. Pain. 2005;119(1-3):16-25. doi:10.1016/J.PAIN.2005.09.008

56. Singh S, Clarke C, Lawendy AR, Macleod M, Sanders D, Tieszer C. A prospective, randomized controlled trial of the impact of written discharge instructions for postoperative opioids on patient pain satisfaction and on minimizing opioid risk exposure in orthopaedic surgery. Curr Orthop Pract. 2018;29(4):292-296. doi:10.1097/BCO.0000000000000632 


\subsection{CHAPTER 4 DISSCUSSION AND DISSEMINATION}

\subsection{SUMMARY OF MAJOR FINDINGS}

The major findings from the study of both the outpatient breast and anorectal surgery populations are as follows: First, patient-reported pain was non-inferior and adequately controlled after implementation of this multipronged, opioid-sparing intervention. Second, patient and provider education, multimodal non-opioid analgesic medications and opioid-reduced prescriptions were reaffirmed to be effective as opioid-reducing interventions. They were feasibly combined together as bundle and implemented at a health-systems level. Third, we reaffirmed that when opioid-reduced or opioid-rescue prescriptions were filled, that actual opioid consumption was still low, with no increase in prescription refills. Finally, we again confirmed that appropriate medication disposal increases with specific instructions; however, overall still remains quite low.

\subsubsection{Patient-reported pain after surgery}

. This patient-centred primary outcome of average pain was reported on a 11-point numeric rating scale (0-10) which was completed at the first post-operative appointment. We showed similar levels of pain control that were non-inferior in the pre- and post-intervention group [(2.0 vs $2.1 / 10, \mathrm{p}=0.40$, breast surgery) and (2.8 vs $2.6 / 10, \mathrm{p}=0.33$, anorectal surgery)].

Furthermore, with the NRS, pain levels are generally interpreted as $0=$ no pain, $1-3=$ mild pain, 4-6 = moderate pain, 7-10 = severe pain. As both anorectal and breast surgery levels were rated between 2-3/10 and a level of 4/10 is defined as inadequate analgesia, we believe that not only was non-inferior pain demonstrated but that pain was indeed adequately controlled in both groups.

\subsubsection{Patient education}

Pain is defined by the International Association for the Study of Pain (IASP) as including two components. First, there is 'the objective, physiologic and sensory aspects' but there is also the 
'subjective, emotional and psychological components.${ }^{1}$ As anxiety and emotional status are important predictors of post-operative pain, education targeting clarification of patient expectations and providing clear analgesic instructions are key factors in managing postoperative pain..$^{2-6}$ This is consequential considering the increasing complexity of surgery and analgesic options.

While adequate analgesia is critical, patients need to understand complete elimination of pain is not a reasonable expectation, and a mild amount of discomfort is normal post-operatively. We believe that our study reaffirms that both verbal and written instructions along with clarification of patient expectations are important aspects of peri-operative care, and likely contributed to overall patient satisfaction and opioid-reduction.

There was variability in who gave the verbal instructions in our group, and this was not captured and the effect of this is unknown. While often it was a general surgeon or surgical resident, there may have other times where a nurse or non-surgical resident were giving the instructions. In particular, the recovery room and day surgery nurses were key in reviewing the written instructions with patients. There was positive feedback from the nurses as the variability of instructions between different surgeons had decreased with the standardized education sheets.

\subsubsection{Provider Education}

The opioid crisis partially stemmed from providers being educated that pain was "the fifth vital sign" and that pain was undermedicated with underestimation of the addiction potential of opioids. Unfortunately, we now need to "undo" this education partially and "re-educate" providers on appropriate opioid prescribing practices and multimodal analgesia.

Buy-in of the surgeons, anesthetists and entire care team was central to the success of the STOP Narcotics initiative. This allowed change at a health systems level, which is arguably the most effective of all opioid-reducing interventions. 
Our study corroborated Wetzel and Howard, reaffirming provider education to be an effective opioid-reducing strategy. ${ }^{78}$ During provider education, feedback was obtained and questions were answered surrounding the STOP Narcotics protocol, which likely assisted with compliance at the departmental level.

Even prior to implementation of the intervention in this pre- post study, opioid prescribing decreased from a median of 30 pills in the retrospective data to 20 pills in the pre-intervention group. This was likely due to increased conversation about the opioid crisis among the care team, discussion of the upcoming pre- and post-intervention study and re-emphasizes the significance of surgeon buy-in and education.

Evidence for opioid-reduction needs to be presented to the entire care team (surgeons, anesthetists, residents, preadmission clinic (PAC) staff and nurses, post-anesthesia care unit (PACU) nurses and day surgery nurses) as these members all have an integral role in patient care and pain management.

We found the most effective education material was based on the following: a) discussing the history of the opioid crisis; b) explaining why the surgeon and care team have a role; c) discussing evidence-based methods of opioid-reduction and multimodal analgesia; d) addressing common concerns - often surrounding prescription refill requests and concerns for inadequate quantity of opioid providing poor analgesia.

Provider education is fundamental and should be the first step in the approach to reducing opioid use as it allows for organizational change and simultaneous development of a multimodal comprehensive pain regimen for patients at the institution.

\subsubsection{Intra- and post-operative medication utilization and prescribing.}

A limited supply of opioids in the form of an opioid "rescue" or "optional" prescription was utilized in this study. An expiry-date of 7 days prevented the filling of the prescription for potential later use. A quantity of 10-opioid pills was chosen as a guideline for these 
prescriptions. This was based on previous studies examining the amount of opioid patients consume after outpatient surgery. ${ }^{910}$ Our study confirmed that in patients who filled their prescription the amount of opioid consumed was small (4 pills) in the post-intervention group. Indeed, the majority of patients (55\%) did not fill their opioid prescription.

An opioid-rescue prescription was utilized in the intervention. If the patient did not live near a pharmacy, there was a discussion between the provider and the patient as to whether the prescription should be filled immediately. We believe this approach was appropriate due to recent guidelines promoting rescue-opioid therapy, in addition to evidence demonstrating equivalent analgesia with non-opioid multimodal measures. ${ }^{81112-14}$

The anesthesia department collaborated on this multidisciplinary plan and were consistently supportive of the STOP Narcotics initiative. The intra-operative protocol recommendations of ketorolac, dexamethasone and ondansetron are based on level 1 evidence and were recommended by our anesthesia department. We did not collect data on the adherence to these recommendations, due to strong current evidence for their use. As well, the majority of our anesthetists reported using these medications routinely prior to the study. Anesthetists have an integral role, especially in controlling immediate post-operative analgesia. We found a short conversation occurring between the anesthetist and surgeon about the intra- and post-operative analgesia plan and the appropriateness of the NSAIDs in the patient was beneficial in providing a comprehensive analgesic plan for each patient.

A prescription for a NSAID was given post-operatively to be used regularly for 72 hours. Meloxicam, which is not an over-the-counter medication in Canada, was routinely used to promote patient compliance. In addition, a prescription NSAID may have additional placebo benefit, however, more evidence is needed to elucidate if this is indeed true. The cost of 6 pills (72 hours) of meloxicam is approximately $\$ 3.00 \mathrm{CAD}$, which is similar to over the counter ibuprofen or naproxen, although there is a pharmaceutical dispensing fee associated with this. There was not a significant number of voiced complaints from patients expressing concern due to the expense of this prescription. Although over-the-counter naproxen was an option in the STOP Narcotics protocol this was utilized by very few surgeons and patients. 
Only $44 \%$ and $45 \%$ of patients in the breast and anorectal surgery groups filled their rescueopioid prescription. Only $9 \%$ of the entire population reported filling it for pain not satisfactorily controlled with regular acetaminophen and NSAIDs. This corroborates evidence that NSAIDs and acetaminophen are effective in post-operative analgesia. Indeed, meta-analyses by the Cochrane Database indicated that ibuprofen and acetaminophen together may be more effective than opioids for acute post-surgical pain. ${ }^{12,13}$ Compliance of taking NSAIDs with acetaminophen in our study was not $100 \%$ and we did not investigate why some patients were non-adherent to the protocol. Possibilities include minimal pain not requiring additional analgesia, an intolerance to the medication or simply non-adherence. There is level 1 evidence for these medications in the peri-operative period and they should be routinely used for patients without contraindications.

We utilized dexamethasone, acetaminophen, NSAIDs and opioid-rescue prescriptions in our opioid-sparing multimodal analgesic strategy. While not part of the data captured, all surgeons at our institution reported routine use of local anesthetic when surveyed. In addition, metronidazole and polyethylene glycol (PEG) were used in the anorectal group. Conservative measures included ice therapy in the breast surgery group and sitz baths in the anorectal surgery group. All these interventions were implemented based on evidence and recommendations from our general surgeons and anesthetists. Other medications such as NMDA antagonists, Alpha-2 agonists, gabapentinoids, selective serotonin reuptake inhibitors, and tricyclic antidepressants were not added to the protocol due to side effect profile, insufficient evidence and minimal analgesic benefit.

We designed our multimodal intra- and post-operative analgesic regimen to be focused on maximizing the pharmacologic benefits, while minimizing adverse effects. ${ }^{15}$ This can be accomplished through the combination of different mechanisms of actions of analgesic drugs that have additive, if not synergistic effects, facilitating the recovery process. These various medications and interventions need to be safe, simple, and cost-effective. 


\subsubsection{Standardized pain care bundle}

"Standardized patient care bundles" are increasingly utilized as methods to implement multiple evidence-based interventions for quality improvement initiatives on a large scale. While every patient requires an individualized approach, our study has shown the value of a standardized bundle to guide surgeons, anesthetists and nurses in providing a comprehensive pain management strategy while reducing opioids in this population. Development of a "pain care bundle" should be a central component to new care pathways, focusing on the minimization of opioid use, and multimodal analgesia

Indeed, while education of the entire care team is important, STOP Narcotics reaffirmed the value of introducing a protocol or guideline at a divisional level. While there is always crossover between written guidelines and provider education, implementing change in the form of a "bundle" at an organizational or departmental level provides the foundation for institutional change.

\subsubsection{Prescription refills}

A concern of surgeons is often increased phone calls for prescription renewals after surgery. Our results demonstrate there was no difference in prescription requests which remained quite low. This was consistent with another study evaluating an intervention in 246 patients where opioid prescribing decreased by 53\%. Refill requests did not change and were required in only $0.4 \%$ of patients. ${ }^{16}$ After reducing opioid prescriptions by $38 \%$ Howard also demonstrated prescription refills did not change significantly from $4.1 \%$ to $2.5 \% .{ }^{17}$ Sekhri examined probability of prescription refill in 26,520 opioid naïve patients who underwent major or minor surgical procedures. ${ }^{18}$ There were $8.7 \%$ of patients who filled their prescription, however, the probability of a refill did not correlate with quantity of opioid prescribed. ${ }^{18}$

With a movement to opioid-rescue prescriptions or potentially discharging a patient without an opioid prescription, there is need for more research on methods to provide a limited supply opioid prescription more conveniently and efficiently if needed. 


\subsubsection{Excess medication disposal}

Even with specific instructions appropriate disposal of excess medication to a pharmacy increased but was still quite low in our study. With Hill reporting only $28 \%$ of opioid prescriptions being consumed, considering there are over 50 million ambulatory procedures in the United States alone, this truly may contribute to an astounding quantity of leftover opioid medication. Data from the National Survey on Drug Use and Health indicates that prescription opioids for non-medical use are most commonly obtained from a friend or relative in the majority of cases. Potential solutions for the tremendous amount of leftover medication and disposal techniques are areas we have identified for future study at our institution.

\subsubsection{Compliance}

We established the role of the surgeon on account of the misuse of prescription opioids, the risks of chronic opioid use, the current practice of variable and excessive opioid prescriptions and the need to instruct the patient on appropriate disposal of excess medication.

A major limitation to the study was the lack of data as to why some patients and staff were noncompliant with the protocol. Therefore, after study completion, we distributed a survey to gather information from surgeons, residents and office administration to gather feedback regarding compliance to the study. We used medication and prescription compliance as an indirect marker of patient and surgeon compliance.

\subsubsection{Patient compliance}

Patients' use of acetaminophen and NSAIDs increased significantly with a prescription NSAID and instructions its utilization. When considering all groups compliance was 70\% for NSAIDs and $79 \%$ for acetaminophen. Medication utilization may be a poor marker for compliance as patients who had very minimal or no pain may not have felt the need to take the medication. When asked why patients in our cohorts filled the rescue-opioid prescription the most common 
reasons were "just in case" or "I was instructed to fill it". The majority of patients (94\%) did find the written instructions helpful.

We did not examine the data for geographic distribution of patients or examine the socioeconomic status of those who filled their prescriptions. We do know from previous data that lower socioeconomic status is a risk factor for misuse of opioids. In particular, the relationship of patients who are filling or not filling their rescue opioid prescription may be correlated to their geography and socioeconomic status, particularly if they lived further away from a 24-hour pharmacy. While this was not a focus of our study, it is an area that may benefit from further study.

\subsubsection{Surgeon compliance}

All but one of the 20 surgeons at our centre changed their opioid prescribing habits. In following prescriptions over 1-year, ongoing compliance and use of the STOP Narcotics initiative in outpatient procedures remains high, and knowledge translation has been witnessed into other procedures not included in the study such as thyroidectomies, laparoscopic appendectomies and other more minor outpatient procedures. When surveyed, the respondents stated they discussed pain control with their patients "usually" or "always" 9/17 (53\%) in clinic and 15/17 (88\%) on the day of surgery. The majority of the surgeons and residents found the post-operative plan on our EMR helpful and easy to use. The surgical residents wrote the majority of prescriptions and reported using the post-operative plan without exception. All surgeons subjectively noticed no difference in phone calls, with the exception of two surgeons (one noticed a decrease and another an increase).

\subsubsection{Office administration}

All executive office administration staff reported no subjective difference in phone calls from patients, with the exception of three (one reporting an increase and two reporting a decrease) when surveyed. 


\subsubsection{Nursing compliance}

As the previous culture at our institution was for every adult patient to receive an opioid prescription after surgery, initially day surgery nurses were instructing patients to fill their rescue opioid prescription immediately. Currently, more than a year after implementation of STOP narcotics our day surgery team is very proud of the role they play and have been involved in making educational videos for other institutions, clearly explaining the concept of the opioidrescue prescription and multimodal analgesia. Interestingly, one factor for which they have expressed ongoing satisfaction is that in standardizing the post-operative pain care protocol, variability in the many written instructions provided by different surgeons has been virtually eliminated.

\subsubsection{Limitations}

While our study has many strengths, it is not without its limitations. With a prospective, observational pre- and post-study there is inherent bias, and with access to retrospective data, we know practice change occurred prior to the implementation date of the intervention. This results in an overall underestimation of the already significant reduction in opioid prescribing. It also emphasizes the significant impact of organizational interventions such as guidelines and provider education.

As this was completed at an academic centre, there is limited external validity with our inclusion criteria. However, with lower rates of resident turn-over and variability in non-academic centres, it is reasonable to hypothesize this may be successful in the community setting. After study completion we implemented this intervention in two regional community hospitals and although most follow-up data was subjective, there were no reports of inferior analgesia from nurses or patients, and positive feedback from nursing was received about standardizing instructions and practice among surgeons. While this is an area for ongoing research, an identified difficulty was having patients complete the modified brief pain inventory survey in a busy surgical clinic to retrieve objective outcomes data. 
There is also limited external validity in regards to other procedures. Although we did not include other minor surgeries in our inclusion criteria, we witnessed spillover into other procedures and believe that with small adjustments it may be successful in other surgical procedures and specialties.

It is difficult to determine causation in a prospective cohort, non-inferiority study. The purpose of this study was not to demonstrate the effectiveness of any specific intervention or medication, as an RCT would be a better model for this. Rather, our purpose was to combine multiple evidence-based, opioid-sparing modalities into a standardized bundle and implement this at a health systems level. It was not feasible to randomize some elements of intervention such as health care provider education. Indeed, it was also not ethical to compare the intervention to placebo or no pain control. If we randomized the intervention to the "standard of care" from retrospective data we knew this was extremely variable. Additionally, this was not designed as an RCT due to a unified belief within the division of general surgery that practice change regarding opioid prescribing was excessive and change needed to occur. There was also concern this pre-existing bias among the surgeons may affect whether the randomization process would be followed appropriately. Additionally, with the intervention implemented at a single point in time, this may have resulted in less variability in surgeons, residents, and nurses as the inclusion criteria, surgical practices, and outcomes such as breast or anorectal pain did not change over time.

The primary outcome of average pain in the first 7 post-operative days was determined at the first post-operative appointment, typically four weeks at our institution. This provides an opportunity for recall bias to affect our outcomes. However, both the pre- and post-intervention groups would have had equal exposure to this bias. In addition, patient-reported pain was not just statistically non-inferior but was very similar in both groups.

The post-intervention group received additional instructions and may have been more aware of our efforts to decrease opioid prescribing. This may have resulted in bias known as the Hawthorne effect - where patients altered their behavior or survey inputs due to their awareness of being observed. This bias may likely minimal as both the pre- and post-intervention patients 
were consented, aware of the research topic, filled out the modified brief pain inventory, and were unaware of whether they were part of the pre- or post-intervention group

Our sample size was adequately powered to assess breast surgery and anorectal surgery outpatient procedures as one group. However, within these procedures there were subgroups and associated variability with regards to expected post-operative pain levels. Some of these subgroups such as axillary lymph node dissection (ALND) or hemorrhoidectomy patients did not reach statistical power, and therefore we cannot reach conclusions in these small groups of patients. Future studies, that examine these subgroups may be useful to design multimodal analgesic strategies specific to these procedures.

While not part of the data captured, all surgeons at our institution reported routine use of local anesthetic when surveyed. In addition, liposomal bupivacaine for possibly more extended analgesia was not available at the time of this study in Canada. Finally, our study does not demonstrate perfect compliance by patients, nurses or surgeons. Early in the intervention some perioperative nurses were still instructing patients to fill their rescue-opioid prescription which was contrary to the patient education given. One surgeon also did not change his/her practice with quantity of OME actually increasing in the post-intervention group. All these patients were included however, in an intention-to-treat analysis as we wanted this intervention to present a real-life quality improvement initiative.

\subsection{DISSEMINATION}

\subsubsection{Dissemination to other centres}

With opioid use in surgery being topically relevant, there was interest in other institutions and surgical specialties in the experience with the STOP Narcotics protocol. As there is limited external validity in our academic, single-centre design we a decided to implement the STOP Narcotics initiative in outpatient general surgical procedures in the community hospitals of St. Thomas, Ontario and Stratford, Ontario. St. Thomas Elgin General Hospital (five full-time general surgeons) and Stratford General Hospital (six full-time general surgeons) are two 
regional community hospitals in southwestern Ontario and both perform high volumes of outpatient general surgery procedures.

These hospitals perform outpatient laparoscopic cholecystectomy, open ventral hernia repair, open inguinal hernia repair, breast surgery and anorectal surgery and similar multipronged protocols and instruction sheets were created specific to their institution and with their hospital practices in mind. In addition, these hospitals created a general "minor surgery" instruction sheet which was used for laparoscopic appendectomy patients, carpal tunnel surgery and other minor procedures they felt appropriate to be included.

A formal educational meeting was held with each of the two groups of regional partners with all of the general surgeons in attendance. An anesthetist from our institution also held morning teaching rounds with their anesthetists to introduce the STOP Narcotics protocol. Implementation was assigned through a few of the surgeons taking on responsibilities at their centre. These surgeons looked after reviewing and distribution of the instruction sheets to ensure all surgeons were satisfied with the post-operative instructions, nursing education of the day surgery and PACU nurses and the office administrators and nurses, and providing education and obtaining support from the anesthesia department for the intervention.

Both centres continued the intervention for 3 months at which time a review was held to assess progress. All surgeons were satisfied with the STOP Narcotics initiative, and subjectively did not notice any change in patient satisfaction or pain control. They did not subjectively notice an increase in phone calls or prescription refills. There had been no resistance, but actual encouragement from the anesthesia department to implement and continue with this initiative, and routinely dexamethasone, ketorolac and ondansetron were being given intra-operatively.

One hospital had electronic prescriptions and the other pre-printed the standardized opioidrescue and meloxicam prescriptions. Both locations did not have difficulty integrating these into their regular practice. The electronic version was more user-friendly once the post-operative prescriptions and instructions had been placed in a package. 
Although we attempted to gather surveys at each post-operative clinic visit from these community hospitals, feedback was unanimous from the surgeons that in a busy clinic, having patients fill out a post-operative pain survey was difficult and cumbersome to the flow of patients. Therefore, we did not reach statistical power in either of these groups so cannot reach conclusions about non-inferior pain control at either hospital.

Other positive feedback included increased nursing satisfaction from the standardization of prescribing practices and post-operative instructions among the surgeons. It was felt this should be a division-wide initiative, with multiple surgeons championing the initiative rather than having a single surgeon lead the initiative as it can be difficult to correct a colleague's practice.

\subsubsection{Dissemination to other surgical specialities}

At London Health Sciences Centre we are currently holding monthly meetings with the surgical specialties of orthopedics, obstetrics and gynecology, urology, otolaryngology, plastic surgery and pediatric anesthesia with both a staff member and resident representing each specialty. We have obtained buy-in for similar initiatives in each of these specialties. Currently, we are in the process of creating similar protocols and instruction sheets which are specific to each of these surgical specialities and procedures.

\subsubsection{Follow up and study dissemination}

The dissemination of this information first started with presentation of the hernia and laparoscopic cholecystectomy work completed prior to this thesis, at the American College of Surgeons Clinical Congress. This was then published in the Journal of the American College of Surgeons. Currently, the work on outpatient breast surgery and anorectal procedures will be presented at the American Society of Breast Surgeons Annual Meeting 2019 and the Annual Scientific Meeting of the American Society of Colon and Rectal Surgeons 2019. This work is also currently submitted to two scientific journals. 
This work has been presented in the following meetings: Canadian Association of General Surgeons Resident Research Meeting, Health Quality Ontario Webinar, Ontario Surgical Quality Improvement Network, Choosing Wisely Canada Meeting, and the Ontario Association of General Surgeons Annual Meeting. The protocol has also been presented at the following hospitals: St. Thomas Elgin General Hospital, Stratford General Hospital, Brampton Civic Hospital, Hanover and District Hospital, Woodstock General Hospital, The Ottawa Hospital (both campuses), Chatham-Kent Health Alliance, Bluewater Health Hospital, Belleville General Hospital, Vancouver General Hospital (Vancouver, BC), Alberta Health Services (Edmonton, AB), and Foothills Medical Centre (Calgary, AB).

This work was also disseminated through the following radio broadcasts and news releases: CTV news London (press release and upcoming interview); Canadian Press Article - Republished by CBC health, The National Post, Ottawa Citizen, Yahoo! News, CTV Toronto, City TV Toronto, Sudbury Star, Victoria Times, Colonist, Kingston Whig-Standard and 8 other smaller newspapers. Also published in the London Free Press, Sarnia Observer, and Stratford BeaconHerald. Radio station broadcasts included AM 980, 1290 CFPL, CBC London and Windsor, CMHL Hamilton, CHED Edmonton, 660 News Calgary, 96.3 Toronto, AM 740 Toronto. Specific hospital press included: Eurekalert Press Release: Hospital News and MedicalXpress.

\subsection{CONCLUSION}

In this quality improvement initiative, the STOP Narcotics was successfully implemented as a multidisciplinary, divisional, multipronged "patient pain care bundle" to institute multiple evidence-based interventions in outpatient breast and anorectal surgery. While every patient requires an individualized approach, our study has shown the value of a standardized bundle, to guide surgeons in ensuring a comprehensive pain control strategy with responsible opioid prescribing. We demonstrated that adequate pain control may be obtained, while significantly decreasing opioid prescribing, and often eliminating opioid utilization by patients, through dedicated health systems change by the entire care team. This decreases the opioid exposure risk in patients and reduces the amount of excess medication which is left for misuse or diversion. Considering the number of outpatient breast and anorectal procedures completed per year, if 
there is continued implementation by other institutions and surgical disciplines this would have a significant impact on the amount of opioid prescribed and play a primary prevention role in the opioid crisis. This also may provide a framework for future analgesia guidelines in outpatient breast and anorectal surgery.

\subsection{REFERENCES}

1. Treede R-D. The International Association for the Study of Pain definition of pain. PAIN Reports. 2018;3(2):e643. doi:10.1097/PR9.0000000000000643

2. Sjöling M, Nordahl G, Olofsson N, Asplund K. The impact of preoperative information on state anxiety, postoperative pain and satisfaction with pain management. Patient Educ Couns. 2003;51(2):169-176. http://www.ncbi.nlm.nih.gov/pubmed/14572947. Accessed January 5, 2019.

3. Sugai DY, Deptula PL, Parsa AA, Don Parsa F. The importance of communication in the management of postoperative pain. Hawaii J Med Public Health. 2013;72(6):180-184. http://www.ncbi.nlm.nih.gov/pubmed/23795326. Accessed January 5, 2019.

4. Egbert LD, Battit GE, Welch CE, Bartlett MK. Reduction of Postoperative Pain by Encouragement and Instruction of Patients. N Engl J Med. 1964;270(16):825-827. doi:10.1056/NEJM196404162701606

5. Langer EJ, Janis IL, Wolfer JA. Reduction of psychological stress in surgical patients. $J$ Exp Soc Psychol. 1975;11(2):155-165. doi:10.1016/S0022-1031(75)80018-7

6. Scott LE, Clum GA, Peoples JB. Preoperative predictors of postoperative pain. Pain. 1983;15(3):283-293. http://www.ncbi.nlm.nih.gov/pubmed/6856324. Accessed January 5, 2019.

7. Wetzel M, Hockenberry J, Raval M V. Interventions for Postsurgical Opioid Prescribing. JAMA Surg. 2018;153(10):948. doi:10.1001/jamasurg.2018.2730

8. Howard R, Waljee J, Brummett C, Englesbe M, Lee J. Reduction in Opioid Prescribing Through Evidence-Based Prescribing Guidelines. JAMA Surg. 2018;153(3):285. doi:10.1001/jamasurg.2017.4436

9. Feinberg AE, Chesney TR, Srikandarajah S, Acuna SA, McLeod RS, Best Practice in Surgery Group. Opioid Use After Discharge in Postoperative Patients. Ann Surg. 2018;267(6):1056-1062. doi:10.1097/SLA.0000000000002591

10. Bicket MC, Long JJ, Pronovost PJ, Alexander GC, Wu CL. Prescription Opioid Analgesics Commonly Unused After Surgery: A Systematic Review. JAMA Surg. 2017;152(11):1066. doi:10.1001/jamasurg.2017.0831 
11. Overton HN, Hanna MN, Bruhn WE, et al. Opioid-Prescribing Guidelines for Common Surgical Procedures: An Expert Panel Consensus. J Am Coll Surg. 2018;227(4):411-418. doi:10.1016/j.jamcollsurg.2018.07.659

12. Toms L, McQuay HJ, Derry S, Moore RA. Single dose oral paracetamol (acetaminophen) for postoperative pain in adults. Cochrane database Syst Rev. 2008;(4):CD004602. doi:10.1002/14651858.CD004602.pub2

13. Derry CJ, Derry S, Moore RA. Single dose oral ibuprofen plus paracetamol (acetaminophen) for acute postoperative pain. Cochrane Database Syst Rev. 2013;(6):CD010210. doi:10.1002/14651858.CD010210.pub2

14. Derry S, Derry CJ, Moore RA. Single dose oral ibuprofen plus oxycodone for acute postoperative pain in adults. Cochrane Database Syst Rev. June 2013. doi:10.1002/14651858.CD010289.pub2

15. Wick EC, Grant MC, Wu CL. Postoperative Multimodal Analgesia Pain Management With Nonopioid Analgesics and Techniques. JAMA Surg. 2017;152(7):691. doi:10.1001/jamasurg.2017.0898

16. Hill M V., Stucke RS, McMahon ML, Beeman JL, Barth RJ. An Educational Intervention Decreases Opioid Prescribing After General Surgical Operations. Ann Surg. 2018;267(3):468-472. doi:10.1097/SLA.0000000000002198

17. Howard R, Waljee J, Brummett C, Englesbe M, Lee J. Reduction in Opioid Prescribing Through Evidence-Based Prescribing Guidelines. JAMA Surg. 2018;153(3):285. doi:10.1001/jamasurg.2017.4436

18. Sekhri S, Arora NS, Cottrell H, et al. Probability of Opioid Prescription Refilling After Surgery. Ann Surg. 2018;268(2):271-276. doi:10.1097/SLA.0000000000002308

19. Jones, Stephen RG. "Was there a Hawthorne effect?." American Journal of sociology 98.3 (1992): 451-468. 


\title{
5.0 CHAPTER 5. APPENDICES
}

\subsection{Appendix 5.1 REB \#109251}

Western

Research Ethics

Research

Western University Health Science Research Ethics Board HSREB Delegated Initial Approval Notice

\author{
Principal Investigator: Dr. Ken Leslie \\ Department \& Institution: Schulich School of Medicine and Dentistry $\backslash$ Surgery,London Health Sciences Centre \\ Review Type: Delegated \\ HSREB File Number: 109651 \\ Study Title: The Standardization of Outpatient Procedure Narcotics: A prospective cohort study to reduce opioid use in \\ outpatient general surgery (The STOP Narcotics Study)
}

HSREB Initial Approval Date: October 18, 2017

HSREB Expiry Date: October 18, 2018

Documents Approved and/or Received for Information:

\begin{tabular}{|l|l|l|}
\hline Document Name & Comments & $\begin{array}{l}\text { Version } \\
\text { Date }\end{array}$ \\
\hline Western University Protocol & Received 12OCT17 & \\
\hline Letter of Information \& Consent & & $2017 / 10 / 16$ \\
\hline $\begin{array}{l}\text { Data Collection Form/Case Report } \\
\text { Form }\end{array}$ & $\begin{array}{l}\text { Numerical Rating Scale - Received } \\
2017 / 09 / 29\end{array}$ & \\
\hline $\begin{array}{l}\text { Data Collection Form/Case Report } \\
\text { Form }\end{array}$ & $\begin{array}{l}\text { Post Operative Pain Survey and Consent } \\
\text { Form. }\end{array}$ & $2017 / 07 / 17$ \\
\hline
\end{tabular}

The Western University Health Science Research Ethics Board (HSREB) has reviewed and approved the above named study, as of the HSREB Initial Approval Date noted above.

HSREB approval for this study remains valid until the HSREB Expiry Date noted above, conditional to timely submission and acceptance of HSREB Continuing Ethics Review.

The Western University HSREB operates in compliance with the Tri-Council Policy Statement Ethical Conduct for Research Involving Humans (TCPS2), the International Conference on Harmonization of Technical Requirements for Registration of Pharmaceuticals for Human Use Guideline for Good Clinical Practice Practices (ICH E6 R1), the Ontario Personal Health Information Protection Act (PHIPA, 2004), Part 4 of the Natural Health Product Regulations, Health Canada Medical Device Regulations and Part C, Division 5, of the Food and Drug Regulations of Health Canada.

Members of the HSREB who are named as Investigators in research studies do not participate in discussions related to, nor vote on such studies when they are presented to the REB.

The HSREB is registered with the U.S. Department of Health \& Human Services under the IRB ragistration number IRB 00000940.

Ethics Otfice, xn behalf of Dr. Joseph Gilbert, HSREB Chair EO: Erika Basile __ Trace Kelly__ Katelyn Harris__ Nicola Morphet__ Karen Gopaul__ Patricia Sargeant 


\subsection{Appendix 5.2 Post-Operative Pain Management Strategy for Anorectal Surgery}

Patients were asked to notify their surgeon if they had a history of stomach ulcers, liver disease, kidney disease or allergies to any of these medications.

First 3 days ( 72 hours) after surgery:

1. Meloxicam $7.5 \mathrm{mg}: 1$ tablet PO, q12 hours, for 3 days (prescription).

2. Acetaminophen $500 \mathrm{mg} ; 1-2$ tablets PO q6hours, for 3 days.

If the patient does not have coverage for Meloxicam, you may prescribe the following:

1. Naproxen $200 \mathrm{mg}$ (Aleve): Take 2 tablets orally, every 12 hours, for 3 days.

To maximize pain relief, it was strongly recommended to take both of these medications.

After 3 days (72 hours) after surgery:

1. Continue Acetaminophen $500 \mathrm{mg}$ : 1-2 tablets PO q6hours PRN.

2. Ibuprofen $400 \mathrm{mg} ; 1$ tablet PO q6hours PRN.

Patients are given separate prescriptions with the following instructions:

Tramadol $50 \mathrm{mg}$ : 1 tab PO q6hours PRN (10 tabs) (Expiry date 7 days)

If the patient does not have coverage for Tramadol, you may prescribe the following.

Codeine $30 \mathrm{mg}: 1$ tab PO q6hours PRN (10 tabs) (Expiry date 7 days)

Patients were given instructions to only fill this prescription, if the above measures do not adequately control their pain.

Metronidazole $500 \mathrm{mg}: 1$ tab PO q12hours for 5 days.

Polyethylene glycol (PEG) $17 \mathrm{~g}$ : Take once to twice daily to avoid constipation. 


\subsection{Appendix 5.3 Multi-pronged Opioid-sparing Intervention for Breast Surgery}

\section{Patient Education:}

Patients were educated by staff, residents, and nurses through written instructions (standardized education sheets) and verbal reinforcement on two occasions: the initial surgical consultation and the day of surgery. Patients' expectations surrounding the surgery, discomfort and the recovery process were clarified. Instructions focused on optimal use of non-opioid analgesic medications (acetaminophen and NSAIDs) and conservative measures such as ice therapy. Patients were instructed to only fill a separate, rescue 10-tablet opioid prescription, if their discomfort was not satisfactorily controlled by other measures. Finally, patients were educated on appropriate medication disposal.

\section{Provider Education:}

Surgeons, anesthetists, residents, post-anesthesia care unit (PACU) nurses, and pre-admission clinic (PAC) staff were educated through divisional rounds, meetings and emails. This education focused on current evidence surrounding opioid use and prescribing after surgery, and the development and commencement of the STOP Narcotics initiative.

\section{Intra-operative Protocol:}

During the surgical safety checklist, it was reinforced with the anesthetist that patients were to receive ketorolac (15-30 $\mathrm{mg} \mathrm{IV}$ ), ondansetron (4-8 $\mathrm{mg} \mathrm{IV})$, and dexamethasone (4-8 $\mathrm{mg} \mathrm{IV}$ ) during the surgery. All surgeons were encouraged to use local anesthetic field blocks.

\section{Post-operative Protocol:}

Patients received a prescription for an NSAID (meloxicam $7.5 \mathrm{mg}$ or naproxen $400 \mathrm{mg}$ ) to be taken twice daily for 72 hours. Acetaminophen $500 \mathrm{mg}$ was also to be taken every 6 hours for 72 hours. After 72 hours patients were instructed to take ibuprofen $400 \mathrm{mg}$ and acetaminophen 500 $\mathrm{mg}$ as needed. A separate rescue prescription of 10-tablets of an opioid prescription was provided (Tramadol $50 \mathrm{mg}$ or Codeine $30 \mathrm{mg}$ ). This prescription expired in 7 days, with instructions to only fill if discomfort was not sufficiently achieved through other analgesic and conservative measures. Ice therapy was also used at the discretion of the surgeon and patient. 


\subsection{Appendix 5.4 Post-operative Pain Management Instructions for Breast Surgery}

Patients were asked to notify their surgeon if they had a history of stomach ulcers, liver disease, kidney disease or allergies to any of these medications.

Ice therapy was suggested as a conservative therapy to help reduce swelling and discomfort of the breast or axilla for the first day after surgery.

First 3 days (72 hours) after surgery:

1. Meloxicam $7.5 \mathrm{mg}: 1$ tablet PO, q12 hours, for 3 days (prescription).

2. Acetaminophen $500 \mathrm{mg}$; 1-2 tablets PO q6hours, for 3 days.

If the patient does not have coverage for Meloxicam, you may prescribe the following:

1. Naproxen $200 \mathrm{mg}$ (Aleve): Take 2 tablets orally, every 12 hours, for 3 days.

To maximize pain relief, it was strongly recommended to take both of these medications.

After 3 days ( 72 hours) after surgery:

1. Continue Acetaminophen $500 \mathrm{mg}$ : 1-2 tablets PO q6hours as needed.

2. Ibuprofen $400 \mathrm{mg} ; 1$ tablet PO q6hours, as needed.

Patients are given separate prescriptions with the following instructions:

Tramadol $50 \mathrm{mg}$ : 1 tab PO q6hours PRN (10 tabs) (Expiry date 7 days)

If the patient does not have coverage for Tramadol, you may prescribe the following.

Codeine 30 mg: 1 tab PO q6hours PRN (10 tabs) (Expiry date 7 days)

Patients were given instructions to only fill this prescription, if the above measures do not adequately control their pain. 
5.5 APPENDIX 5.5 THE STANDARDIZATION OF OUTPATIENT PROCEDURE NARCOTICS: A PROSPECTIVE NON-INFERIORITY STUDY TO REDUCE OPIOID USE IN OUTPATIENT GENERAL SURGICAL PROCEDURES. 


\author{
Dr. Luke Hartford \\ PGY3 General Surgery \\ CURRICULUM VITAE
}

\title{
Component 1:
}

Academic Background and Training:

Biological Sciences - Laurentian University

2005-2007

Doctor of Veterinary Medicine - Ontario Veterinary College

2007-2011

Doctor of Medicine - Northern Ontario School of Medicine

2012-2016

General Surgery - Western University

2016-2021

Opioid Stewardship Council Resident Representative

2018-2021

Resident Training Committee Representative

2019-2021

ATLS Instructor

2017-ongoing

Surgical Interest Group Mentor

2017-ongoing

Awards and Distinctions:

London Health Sciences Centre Physician Safety Award 2018

Resident selected for CAGS General Surgery Resident Research Retreat 2018

Best General Surgery Junior Research Award 2018

Society for Vascular Surgery Scholarship 2015

Dean's Honour's List 2005-2016

Graduated top 15\% class of Veterinary Medicine 2007-2011

American College of Veterinary Pathology Scholarship 2011

American College of Veterinary Ophthalmologists Scholarship 2011

Barb Crowe \& Don Kuzik Memorial Scholarship 2011

(For exceptional commitment to teamwork and willingness; supporting colleagues and classmates)

Top communication skills award in simulated client-based learning 2008

Laurentian University In-Course Scholarship for grade average 2005-2007

\section{Component 2:}

\section{Journal Publications:}

Hartford L, Doucet V, Ramkumar J, Leslie K, Shum J, Asai K. The impact of a hepatopancreatobiliary program on management of pancreatic cancer at Health Sciences North. Canadian Journal of Surgery (2019) Canadian Journal of Surgery (June - Online edition)

Hartford L, Van Koughnett J, Murphy P, et al The Standardization of Outpatient Procedure Narcotics: A Prospective Health Systems Intervention to Reduce Opioid Use in Ambulatory Breast Surgery. (2019) Annals of Surgical Oncology. In Press.

Hartford L, Van Koughnett J, Murphy P, et al The Standardization of Outpatient Procedure Narcotics: A Prospective Non-inferiority Study to Reduce Opioids in Outpatient General Surgical Procedures. Journal of the American College of Surgeons. (2019) Journal of the American College of Surgeons 228(1), 81-88. 
Schick B, Hartford L, Leslie K, Parfitt J, Periampullary Gangliocytic Paraganglioma with Lymph Node Metastases: A Case Report. Canadian Journal of Pathology 2017, Vol 9, Iss 2. Pg 60 .

Hartford L, Sherazadishvili A, Leslie K. Duodenal gangliocytic paraganglioma with lymph node metastasis: A systematic review. International Journal of Hepatobiliary and Pancreatic Disease 2017, Vol 7. Pg 53.

Hartford L, Sherazadishvili A, Leslie K, Parfitt J. A Systematic Review of the Histopathology and Immunochemistry in Duodenal Gangliocytic Paragangliomas with Lymph Node Metastases. Journal of Gastroenterology, Hepatology, \& Digestive Disorders 2018. Currently in press.

\section{$\underline{\text { Abstracts Presented (Presenter underlined): }}$}

1. Hartford L, Tuinema J, Euler K. Ambulance Call Volumes and the Social Determinants of Health, in Timmins, Ontario. (2015).

2. Hartford L, Caswell J. Characterization of an Unknown Alveolar Filling Disorder in the Canine Lung. Summer Leadership and Research Journal, Guelph, ON. (2009)

3. Hartford L, Cruz A, Hurtig M. Non-Invasive Surveillance of Cannon Bone and Joint Health in Racehorses by Quantitative Ultrasound Velocity and Biomarkers. Summer Leadership and Research Journal, Guelph, ON. (2008) 